\title{
Nonequilibrium Steady State and Heat Transport in Nonlinear Open Quantum Systems: Stochastic Influence Action and Functional Perturbative Analysis
}

\author{
Jing Yang \\ Department of Physics and Astronomy, University of Rochester, Rochester, New York 14627, USA \\ Jen-Tsung Hsiang \\ Center for High Energy and High Field Physics, National Central University, Chungli 32001, Taiwan \\ Center for Particle Physics and Field Theory, Department of Physics, Fudan University, Shanghai 200438, China
}

Andrew N. Jordan

Department of Physics and Astronomy, University of Rochester, Rochester, New York 14627, USA

Institute for Quantum Studies, Chapman University, 1 University Drive, Orange, CA 92866, USA

B. L. Hu

Joint Quantum Institute and Maryland Center for Fundamental Physics, University of Maryland, College Park, Maryland 20742, USA

\begin{abstract}
In this paper, we show that a nonequilibrium steady state (NESS) exists at late times in open quantum systems with weak nonlinearity by following its nonequilibrium dynamics with a perturbative analysis. We consider an oscillator chain containing three-types of anharmonicity: cubic $\alpha$ - and quartic $\beta$-type Fermi-Pasta-Ulam-Tsingou (FPUT) nearest-oscillator interactions and the on-site (pinned) Klein-Gordon (KG) quartic self-interaction. Assuming weak nonlinearity, we introduce a stochastic influence action approach to the problem and obtain the energy flows in different junctures across the chain. The formal results obtained here can be used for quantum transport problems in weakly nonlinear quantum systems. For $\alpha$-type anharmonicity, we observe that the first-order corrections do not play any role in the thermal transport in the NESS of the configuration we considered. For KG and $\beta$-types anharmonicity, we work out explicitly the case of two weakly nonlinearly coupled oscillators, with results scalable to any number of oscillators. We examine the late-time energy flows from one thermal bath to the other via the coupled oscillators, and show that both the zeroth- and the first-order contributions of the energy flows become constant in time at late times, signaling the existence of a late-time NESS to first order in nonlinearity. Our perturbative calculations provide a measure of the strength of nonlinearity for nonlinear open quantum systems, which may help control the mesoscopic heat transport distinct from or close to linear transport. Furthermore, our results also give a benchmark for the numerical challenge of simulating heat transport. Our setup and predictions can be implemented and verified by investigating heat flow in an array of Josephson junctions in the limit of large Josephson energy with the platform of circuit QED.
\end{abstract}

Keywords: Nonequilibrium steady state; Anharmonic chain; Feynman-Vernon influence functional; Functional perturbation; Quantum transport; Open quantum systems

Email addresses: jyang75@ur.rochester.edu (Jing Yang), cosmology@gmail.com (Jen-Tsung Hsiang), jordan@pas. rochester.edu (Andrew N. Jordan), blhu@umd.edu (B. L. Hu) 


\section{Contents}

1 Introduction $\quad \mathbf{3}$

1.1 Quantum transport of nonlinear systems - numerically challenging . . . . . . . . . . . . 3

1.2 Analytical results, even perturbative, are helpful . . . . . . . . . . . . . . . . . 4

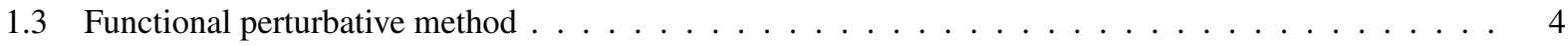

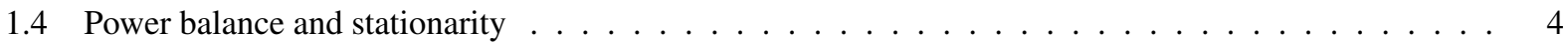

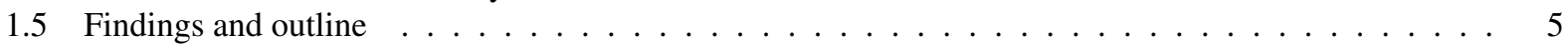

2 The general formalism $\quad \mathbf{5}$

2.1 The Feynman-Vernon formalism for a network of oscillators and baths . . . . . . . . . . . . 5

2.2 The energy exchange . . . . . . . . . . . . . . . . . . . 7

3 The zeroth-order of the heat exchange between an oscillator and their private baths 9

4 The first-order corrections $\quad 13$

4.1 Stochastic generating functional $\mathcal{Z}_{\xi}^{(1)}(0) \ldots \ldots \ldots \ldots \ldots \ldots \ldots$

4.2 Heat exchanges $P_{\xi_{n}}^{(1)}\left(t_{f}\right), P_{\gamma_{n}}^{(1)}\left(t_{f}\right)$ between the oscillator and its private bath $\ldots \ldots \ldots 13$

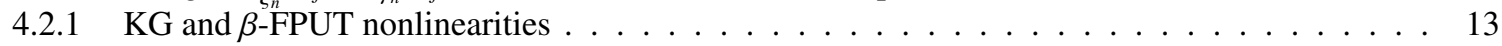

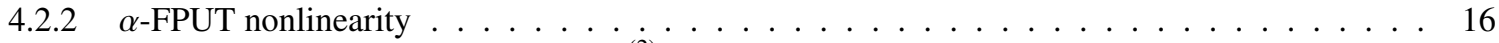

4.3 First order correction to the energy current $P_{v \rightarrow n}^{(2)}\left(t_{f}\right) \quad \ldots \ldots \ldots \ldots$

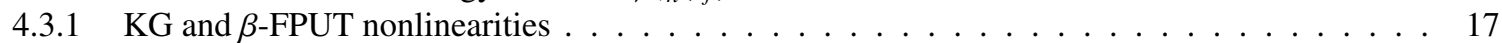

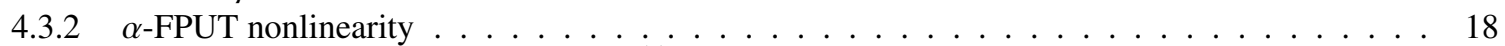

4.4 The first order correction to the energy flux $P_{v \rightarrow n}^{(\eta)}\left(t_{f}\right)$ for $\eta \geq 3 \ldots \ldots \ldots$

5 Diagrammatic representations $\quad \mathbf{2 1}$

5.1 Properties of causal propagator $\tilde{\mathfrak{D}}(\omega) \ldots \ldots \ldots \ldots \ldots \ldots \ldots$

5.2 Feynman diagrams in the time domain . . . . . . . . . . . . . . . . . . . . 22

5.3 Feynman diagrams in the frequency domain . . . . . . . . . . . . . . . . . 23

6 The non-equilibrium steady state (NESS) 2

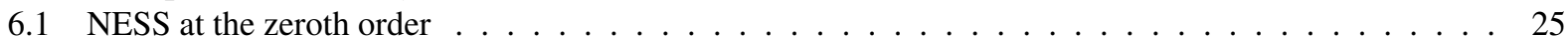

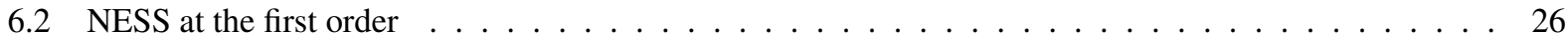

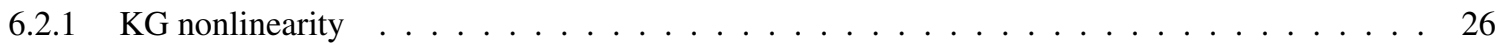

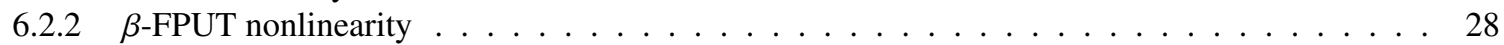

7 Discussion and Conclusion $\quad 30$

8 Acknowledgement $\quad 31$

Appendix A Feynman-Vernon influence functional formalism 31

Appendix B $\quad$ Functional method $\quad 32$

Appendix C Explicit evaluation of the noninteracting stochastic propagating function 34

Appendix D Theorems on the functional derivatives $\quad 36$

Appendix E Useful identities for proving the NESS at the first order 41 


\section{Introduction}

Inasmuch as the equilibrium state of a system in contact with one heat bath is of fundamental importance in the conceptualization and application of the powerful canonical ensemble in statistical thermodynamics, the existence of a nonequilibrium steady state (NESS) is of similar importance for the understanding of nonequilibrium processes which are widely present in Nature. For example NESS is the arena in the discovery of the fluctuation theorems of various forms from Gallivotti and Cohen [1-5] to Jarzynsky and Crook [6-10] and the basis for the investigation of quantum thermodynamics (e.g., [11-14]). One common feature of the equilibrium state and nonequilibrium steady state is their ubiquitous appearance-many, but not all, systems approach an equilibrium or steady state given sufficient time. While the exceptions are not so easily captured but of special interest, the "norm" also needs to be proven or demonstrated for different types of systems and their environments before one can use the many attractive features of NESS to exert control of nonequilibrium systems in such states.

For classical many-body systems the existence and uniqueness of NESS have been studied by mathematical physicists in statistical mechanics for decades. For Gaussian systems (such as a chain of harmonic oscillators with two heat baths at the two ends of the chain) [15] and anharmonic oscillators under general conditions [16] there are definitive answers in the form of proven theorems. Answering this question for quantum many-body systems is not so straightforward. This is the challenge two of the present authors have undertaken [17], showing the existence of NESS at late times for a quantum harmonic chain with two ends coupled linearly to two separate heat baths at different temperatures. (See also [18]). The goal of this work, is to demonstrate the existence of NESS for a system of two weakly nonlinearly coupled (generalizable to a chain of ) quantum oscillators linearly coupled to two heat baths of different temperatures. The forms of interaction amongst the system oscillators correspond to the Fermi-Pasta-Ulam-Tsingou (FPUT) $\alpha$ (cubic nearest neighbor interaction), $\beta$ (quartic nearest neighbor interaction) $[19,20]$ or the Klein-Gordon (KG)('pinned', or quartic self-interaction) [21,22] models. The current work approaches to this problem using a stochastic influence action which is a part of the functional techniques systematically developed in [23-26] and applied to transport problems in [17, 27]. A fluctuation-dissipation relation is shown to exist in such open nonlinear quantum systems by nonperturbative methods in [28]. In this paper using the stochastic influence action, we aim to demonstrate the existence of a NESS at late times for the FPUT and KG models with weak nonlinearity.

In terms of physical contexts and applications, we give a brief sketch of the problem of quantum transport in nonlinear systems, motivating the usefulness of analytic results, perturbative notwithstanding. We then give a brief summary of the functional perturbative method developed in [29] and a description of our approach. We illustrate this by calculating the energy transfer between the weakly nonlinear system and its environment, showing that at late times indeed they balance to first order of nonlinearity, signifying the existence of a NESS. Further background descriptions can be found in the Introductions of [17] and [27].

\subsection{Quantum transport of nonlinear systems - numerically challenging}

Quantum transport is an important class of problems where the nonequilibrium evolution of an open quantum system has been followed and applicable laws examined. A well-known case is the Fourier law for heat conduction in low-dimensional lattices [30, 31]. Amongst the vast literature on this subject suffice it for our purpose here to mention three reviews [32-34]. As for methodologies closest to ours, we mention the density matrix approach (e.g., [35]), the nonequilibrium Green function approach (e.g., [36-39]), the stochastic path integral developed in the context of charge transport in mesoscopic systems [40, 41], the closed time path (CTP, Schwinger-Keldysh, or 'in-in') [42-44], the influence functional [45-47] and the stochastic influence action formalisms [23-26] which are the methods of choice for two of the present authors.

Transport problems depend heavily on numerical computations (e.g., [48]), which enable one to identify qualitatively different behaviors in different regimes. Many analytical approaches, e.g., Boltzmanm molecular dynamics [49], nonlinear kinetic theory and fluctuating hydrodynamics [50] when combined with numerical methods give reasonably good predictions for classical systems in different parameter regimes. However, for quantum many-body systems, numerical computations are a lot more difficult to carry out. For example, even casting the Langevin equations for non-linear quantum systems suitable for numerical analysis poses a challenge [51].

For this reason analytical results for quantum nonlinear systems, even perturbative in nature, may serve some function, in bridging the gaps in the parameter space regions these theories left behind [52]. 


\subsection{Analytical results, even perturbative, are helpful}

The motivation for our investigation using perturbative methods is to explore how analytical methods and results, no matter how unimpressive they are in the face of full scale numerical computations, can still serve some function in clarifying the issues behind energy or charge transport in nonequilibrium weakly-nonlinear systems.

In recent years, several discoveries highlighted the importance of even weak nonlinearity in the thermodynamic and transport properties of classical many-body systems. E.g., it is shown that in the thermodynamic limit a onedimensional (1D) nonlinear lattice can always be thermalized for arbitrarily small nonlinearity [53]. On the effects of nonlinear interaction on disordered chains there is a tentative suggestion that weak nonlinearity can destroy Anderson localization [54]. Thermalization is an important current subject in quantum many-body physics. It is found that even weak nonlinearity can play a pivotal role for thermalization. Thus, a different perspective from nonlinear quantum open systems is desirable. Thermalization via wave turbulence theory has also generated renewed interest in the FPUT and KG models $[55,56]$. These recent developments provided good impetus for us to implement our program on nonequilibrium dynamics of open quantum nonlinear systems. The first step is to explore the conditions for systems in popular models to reach a NESS. Because knowing that a system enters such a state from following its nonequilibrium dynamics can offer great simplification in the analysis of the nonlinear system's long time behavior.

\subsection{Functional perturbative method}

We use the same mathematical framework as in [17], namely, the path-integral [42, 43], influence functional [45, 46] formalism, under which the influence action, the coarse-grained effective action [23] and the stochastic influence action $[24,25]$ are defined. The stochastic equations such as the master equation (see, e.g., [47]) and the Langevin equations (see, e.g., [26]) can be obtained from taking the functional variations of these effective actions. We then invoke the functional perturbative approach of [29] developed further in [27] to treat systems with weak nonlinearity. In this approach we first introduce external sources to drive a linear (harmonic oscillator) system and calculate the in-in generating functional. Taking the functional derivatives with respect to the sources gives the expectation values of the covariance matrix elements or the two-point functions of the canonical variables. The perturbative correction to the two-point functions, or the expectation values of quantum observables due to the nonlinear potential can also be found order by order by taking the appropriate functional derivatives of the generating functional of the linear system linked to external sources.

Going beyond the stochastic path integrals [57, 58] which unravels the effect of a Markovian bath with continuous weak measurement, in this work we unravel the full effect of the bath without invoking the Markov approximation but at the price that the unravelled trajectory is fictitious which may not correspond to any real measurement. It has been shown that the stochastic unraveling admits a time-local master equation [59] even in the presence of the nonlinearity of the quantum open systems. In our stochastic influence action approach, we can define the heat flow rigorously among the oscillators for our cases where the system Hamiltonian contains $\alpha, \beta$-FPUT and KG nonlinearities. Furthermore, we introduce an additional external source $\boldsymbol{h}$, which allows one to compute correlation functions involving momentum operators using the stochastic influence action. These new ingredients allow us to compute steady-state heat currents in a more efficient and economic way than [27], which provides fully nonequilibrium evolution from the transient to the relaxation to a steady state of an open nonlinear system.

\subsection{Power balance and stationarity}

Instead of seeking mathematical proofs for the existence and uniqueness of NESS in quantum nonlinear systems, which to us is a daunting challenge, we aim at solving for the nonequilibrium dynamics of the model systems mentioned above, examine how they evolve in time and see if one or more NESS exist(s) at late times. This is the same approach used earlier by two of the present authors in [17]. Namely, taking the functional variation of the stochastic influence action yields the quantum stochastic equations. The Langevin equation is then used to obtain expressions for the energy flow from one bath to another through the nonlinear system. We can check if these two energy fluxes reach a steady state, and an energy flow (power) balance relation exists. The main result of this work is that at late times at least to first order in the nonlinear interaction between the oscillators, perturbations do not grow any faster or stronger so as to overtake and disrupt the contributions of the linear (harmonic) order. Thus the NESS is explicitly demonstrated to exist for the weakly nonlinear systems studied in our models. 


\subsection{Findings and outline}

Based on the perturbative formalism in the framework of quantum open systems, we give formal expressions for the energy currents up to the first-order of the nonlinear couplings for an FPUT and KG chain. It consists of nonlinearly coupled anharmonic oscillators via the $\alpha$-, $\beta$-FPUT and KG nonlinearities. Each oscillator also linearly couples to its own private bath. We find for $\alpha$-FPUT nonlinearity, the first-order corrections to the energy currents vanish. We demonstrate that for the two-oscillator chain, the NESS is established in the late time up to the first-order of the nonlinear coupling constant. This procedure can be straightforwardly extended to a chain of arbitrary length. In addition, we devise diagrammatic representations for the energy currents in the NESS, which may provide an intuitive understanding of the heat transport at NESS. As a physical application, the ratio between the first-order and zeroth correction of the energy currents in the steady-state provides a measure of the strength of the nonlinearity in the context of thermal transport, which may be applied to controlling thermal transport either distinct from or close to the linear transport. Our analytical results suggest that when the coupling between the chain and the baths is strong or when the temperature bias across the chain is large, the heat transport behaves like a linear one in the presence of the nonlinearities, offering a wider latitude to manipulate nonlinear couplings. Our results can provide a useful benchmark for the numerical simulations of anomalous heat transport in low dimensions in the weakly nonlinear regime [32]. Our setup can be implemented by e.g. engineering an array of Josephson junctions in circuit quantum electrodynamics in the large Josephson energy limit $[60,61]$.

This paper is organized as follows: in Sec. 2 we first present the general formalism of the influence functional and functional perturbation, aiming at the problem of heat transport in a network of oscillators and baths. As a first application of the functional perturbation formalism, in Sec. 3, we apply the functional perturbation formalism to compute the zeroth-order steady-state energy currents in a chain configuration with two oscillators and two baths. In Sec. 4, we compute the first-order correction for the KG, $\beta$-FPUT and $\alpha$-FPUT models respectively. Upon replacing causal propagator for two-oscillator and two-bath configuration with the one for the general $N$-oscillator and $N$-bath configuration, the results in Sec. 3 and 4 can be generalized straightforwardly to the case of a chain of anharmonic oscillators. In Sec. 5, we give the diagrammatic representation of all the energy currents, which provides an intuitive understanding of the heat transport up to the first-order. In Sec. 6, we prove for the two-oscillator two-bath configuration that, the NESS exists at the late times for both KG and $\beta$-FPUT nonlinearities. In Sec. 7, we discuss the possible physical applications of our perturbative results and summarize the findings.

\section{The general formalism}

\subsection{The Feynman-Vernon formalism for a network of oscillators and baths}

We consider $N$ coupled oscillators interacting with their private thermal baths, as shown in Fig. 1. Each oscillator couples to only its nearest neighbors via the linear and the nonlinear mutual couplings, so that these oscillators form a general FPUT chain threading through thermal baths of various temperatures. The dynamics of this entire system can be described by the action

$$
S_{\text {tot }}=S+S_{\phi}+S_{I} .
$$

The action $S_{\chi}$ for the chained oscillators contains linear and nonlinear mutual couplings between the neighboring oscillators

$$
S_{\chi}[\chi]=\int d t\left[\sum_{n=1}^{N} L_{n}-\sum_{n=1}^{N-1} V_{n, n+1}\right],
$$

in which the Lagrangian $L_{n}$ of the $n^{\text {th }}$ oscillator of mass $m_{n}$ takes the form

$$
L_{n}=\frac{1}{2 m_{n}} \dot{\chi}_{n}^{2}-V\left(\chi_{n}\right),
$$

and $\chi_{n}$ denote the oscillator's displacement. The site potential $V\left(\chi_{n}\right)$ may include nonlinear self-interaction of the oscillator. The mutual coupling between two neighboring oscillators $n$ and $n+1, V_{n, n+1}$, takes the form

$$
V_{n, n+1}=\sum_{\eta=2} \frac{\lambda_{\eta}}{\eta}\left(\chi_{n}-\chi_{n+1}\right)^{\eta} .
$$




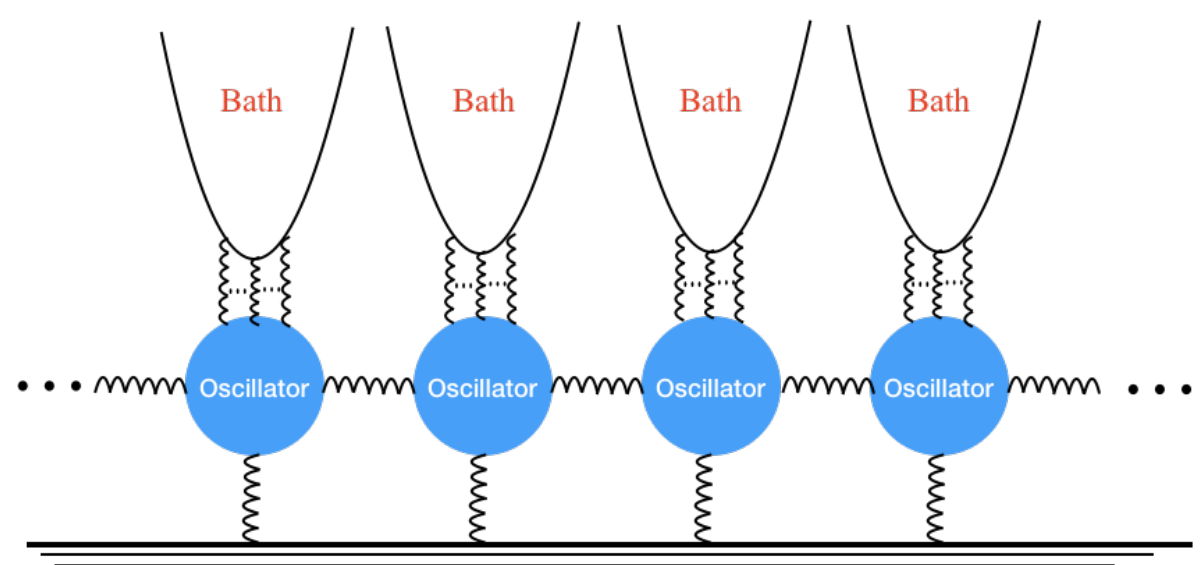

Figure 1: $N$-oscillators represented by blue solid circles that are coupled to their individual thermal baths represented by curved lines, which are theoretically modeled as massless scalar field theory. The inter-oscillator coupling in general could be both linear and nonlinear while the coupling between the chain and the bath is assumed to be linear. The coupling between the oscillators with the substrate contains the linear as well as nonlinear site potential.

If $V\left(\chi_{n}\right)$ contains only the harmonic site potential, then the $\eta=2$ case in fact describes a linear chain, whose nonequilibrium dynamics and the existence of the NESS have been discussed with great details in [17]. Thus, Eq. (2) generalize the previous consideration to include a nonlinear site potential, as well as nonlinear intra-oscillator couplings.

The private baths associated with each oscillator are modeled by independent free massless scalar fields, initially prepared in their respective thermal states. They can have different initial temperatures in general, and their action is given by

$$
S_{\phi}[\boldsymbol{\phi}]=\int d^{4} x \sum_{n=1}^{N} \partial_{\mu} \phi_{n}(\boldsymbol{x}, t) \partial^{\mu} \phi_{n}(\boldsymbol{x}, s),
$$

where $\phi_{n}$ denotes the private bath attached to the $n^{\text {th }}$ oscillator. The interaction between the oscillator and its bath takes the bilinear form

$$
S_{I}[\chi, \phi]=\int d t \sum_{n=1}^{N} e_{n} \chi_{n}(t) \phi_{n}(z(t), t) .
$$

The parameter $z(t)$ describes the trajectory of each oscillator, but we assume that the oscillators are held at fixed position in our subquent treatment. The coupling strength $e_{n}$ between the $n^{\text {th }}$ oscillator and its private bath may take on different values and are not restricted to weak coupling. In addition, to avoid cluster of notations, we introduce the matrix representation such that, say, $\chi=\left(\chi_{1}, \ldots, \chi_{N}\right)^{T}$ to describe $N$ oscillator as a whole.

We will investigate the nonequilibrium dynamics of this anharmonic chain in the framework of quantum open systems, where the oscillator chain is the (reduced) system of our interest and the private baths serve as the environment. The dynamics of the system is fully governed by the reduced density matrix $\rho$, whose evolution can be described by the propagating function $J\left(\chi_{f}, \chi_{f}^{\prime}, t_{f} ; \chi_{i}, \chi_{i}^{\prime}, t_{i}\right)$. It essentially maps the reduced density matrix of the initial configuration at time $t_{i}$ to the reduced density matrix at final time $t_{f}$ according to

$$
\rho\left(\chi_{f}, \chi_{f}^{\prime}, t_{f}\right)=\int d \chi_{i} d \chi_{i}^{\prime} J\left(\chi_{f}, \chi_{f}^{\prime}, t_{f} ; \chi_{i}, \chi_{i}^{\prime}, t_{i}\right) \rho\left(\chi_{i}, \chi_{i}^{\prime}, t_{i}\right)
$$

where $\rho\left(\chi, \chi^{\prime}, t\right) \equiv\left\langle\chi|\hat{\rho}(t)| \chi^{\prime}\right\rangle$ is the elements of the reduced density matrix in the position representation. With the help of the Feynman-Vernon influence functional formalism and the Hubbard-Stratonovich transformation [see Appendix A], the propagating function $J\left(\chi_{f}, \chi_{f}^{\prime}, t_{f} ; \chi_{i}, \chi_{i}^{\prime}, t_{i}\right)$ can be formally expressed as the ensemble average

$$
J\left(\chi_{f}, \chi_{f}^{\prime}, t_{f} ; \chi_{i}, \chi_{i}^{\prime}, t_{i}\right)=\int \mathcal{D} \xi P[\xi] \mathcal{J}_{\xi}\left(\boldsymbol{r}_{f}, \boldsymbol{q}_{f}, t_{f} ; \boldsymbol{r}_{i}, \boldsymbol{q}_{i}, t_{i}\right)
$$


where $P[\xi]$ is the probability distribution functional of the stochastic trajectory, which may be interpreted as the manifestation of quantum fluctuations of the bath fields, and $\mathcal{J}_{\xi}\left(\boldsymbol{r}_{f}, \boldsymbol{q}_{f}, t_{f} ; \boldsymbol{r}_{i}, \boldsymbol{q}_{i}, t_{i}\right)$ is the propagating function for each realization of the stochastic noise $\boldsymbol{\xi}$. Here we have introduced the center-of-mass coordinate $\boldsymbol{r}$ and relative coordinate $\boldsymbol{q}$ by

$$
\boldsymbol{q}(s)=\chi(s)-\chi^{\prime}(s), \quad \boldsymbol{r}(s)=\frac{1}{2}\left[\chi(s)+\chi^{\prime}(s)\right],
$$

respectively. From now on, we will set $t_{i}=0$ without loss of generality and suppress the argument $t_{i}$ in relevant quantities.

The stochastic trajectory $\boldsymbol{\xi}(s)$ satisfies the Gaussian statistics, and its first two moments are

$$
\left\langle\left\langle\xi_{k}(s)\right\rangle\right\rangle=0, \quad\left\langle\left\langle\xi_{k}(s) \xi_{l}\left(s^{\prime}\right)\right\rangle\right\rangle=G_{H}^{(k)}\left(s, s^{\prime}\right) \delta_{k l},
$$

where $G_{H}^{(n)}\left(s, s^{\prime}\right)$ is the noise kernel of the $n^{\text {th }}$ private thermal bath. The stochastic propagation function thus enables us to introduce the stochastic density operator in the same fashion as (7)

$$
\varrho_{\xi}\left(\boldsymbol{r}_{f}, \boldsymbol{q}_{f}, t_{f}\right) \equiv \int d \boldsymbol{r}_{i} d \boldsymbol{q}_{i} \mathcal{J}_{\xi}\left(\boldsymbol{r}_{f}, \boldsymbol{q}_{f}, t_{f} ; \boldsymbol{r}_{i}, \boldsymbol{q}_{i}\right) \varrho\left(\boldsymbol{r}_{i}, \boldsymbol{q}_{i}\right)
$$

such that

$$
\begin{aligned}
\mathcal{J}\left(\boldsymbol{r}_{f}, \boldsymbol{q}_{f}, t_{f} ; \boldsymbol{r}_{i}, \boldsymbol{q}_{i}\right) & \left.=\left\langle\mathcal{J}_{\xi}\left(\boldsymbol{r}_{f}, \boldsymbol{q}_{f}, t_{f} ; \boldsymbol{r}_{i}, \boldsymbol{q}_{i}\right)\right\rangle\right\rangle, \\
\varrho\left(\boldsymbol{r}_{f}, \boldsymbol{q}_{f}, t_{f}\right) & =\left\langle\left\langle\varrho_{\xi}\left(\boldsymbol{r}_{f}, \boldsymbol{q}_{f}, t_{f}\right)\right\rangle\right\rangle,
\end{aligned}
$$

where $\langle\langle\bullet\rangle$ denotes the ensemble average over the probability functional $P[\boldsymbol{\xi}]$.

In the context of the thermal transport along the oscillator chain, we are primarily concerned with the nature of the thermal flow along the chain, and the energy exchange between the chain and the bathes. In next section, we will discuss the physical quantities of our interest for the current configuration.

\subsection{The energy exchange}

We use the master equation developed in [59], which is time-local for general system Hamiltonian, to define the energy exchange between an oscillator and its private bath or its nearest neighbors. The Hamiltonian operator of the chain, corresponding to the action $S_{\chi}$, is

$$
\hat{H}_{\chi}=\sum_{n=1}^{N} \hat{H}_{n}+\sum_{n=1}^{N-1} \hat{V}_{n, n+1},
$$

where the Hamiltonian operator $\hat{H}_{n}$ at site $n$ takes the form

$$
\hat{H}_{n}=\frac{\hat{p}_{n}^{2}}{2 m_{n}}+V\left(\hat{\chi}_{n}\right)
$$

For Ohmic baths, it can be shown that, the evolution of the chain associated with each trajectory $\boldsymbol{\xi}$ can be described by the stochastic Liouville-von Neumann equation [59]

$$
\dot{\hat{\rho}}_{\xi}\left(t_{f}\right)=-i\left[\hat{H}_{\chi}, \hat{\rho}_{\xi}\left(t_{f}\right)\right]+i \sum_{n=1}^{N} \xi_{n}\left(t_{f}\right)\left[\hat{\chi}_{n}, \hat{\rho}_{\xi}\left(t_{f}\right)\right]-i \sum_{n=1}^{N} \gamma_{n}\left[\hat{\chi}_{n},\left\{\hat{p}_{n}, \hat{\rho}_{\xi}\left(t_{f}\right)\right\}\right]
$$

where $\gamma_{n}=e_{n}^{2} /\left(8 \pi m_{n}\right)$. We will calculate $\operatorname{Tr}\left\{\dot{\hat{\rho}}_{\xi}\left(t_{f}\right) H_{n}\right\} / \operatorname{Tr}\left\{\hat{\rho}\left(t_{f}\right)\right\}$, which is the the change of internal energy of oscillator $n$ associated with the trajectory $\xi$, explicitly to identify the all the relevant heat exchanges at site $n$. To this end, we find

$$
\operatorname{Tr}\left\{\hat{\rho}_{\xi}\left(t_{f}\right) \hat{H}_{n}\right\}=i \operatorname{Tr}\left\{\left[\hat{H}_{\chi}, \hat{H}_{n}\right] \hat{\rho}_{\xi}\left(t_{f}\right)\right\}-i \xi_{n}\left(t_{f}\right) \operatorname{Tr}\left\{\left[\hat{\chi}_{n}, \hat{H}_{n}\right] \hat{\rho}_{\xi}\left(t_{f}\right)\right\}+i \gamma_{n} \operatorname{Tr}\left\{\left\{\hat{p}_{n},\left[\hat{\chi}_{n}, \hat{H}_{n}\right]\right\} \hat{\rho}_{\xi}\left(t_{f}\right)\right\},
$$


where we have used the following identities

$$
\operatorname{Tr}\{[A, B] C\}=-\operatorname{Tr}\{[A, C] B\}, \quad \operatorname{Tr}\{[C,\{A, B\}] D\}=-\operatorname{Tr}\{\{A,[C, D]\} B\} .
$$

Carrying out the commutators and anti-commutators in Eq. (17), we find

$$
\begin{aligned}
\operatorname{Tr}\left\{\left[\hat{\chi}_{n}, \hat{H}_{n}\right] \hat{\rho}_{\xi}\left(t_{f}\right)\right\} & =\frac{i}{m_{n}} \operatorname{Tr}\left\{\hat{p}_{n} \hat{\rho}_{\xi}\left(t_{f}\right)\right\}, \\
\operatorname{Tr}\left\{\left\{\hat{p}_{n},\left[\hat{\chi}_{n}, H_{n}\right]\right\} \hat{\rho}_{\xi}\left(t_{f}\right)\right\} & =\frac{2 i}{m_{n}} \operatorname{Tr}\left\{\hat{p}_{n}^{2} \hat{\rho}_{\xi}\left(t_{f}\right)\right\}, \\
\operatorname{Tr}\left\{\left[\hat{H}_{\chi}, \hat{H}_{n}\right] \hat{\rho}_{\xi}\left(t_{f}\right)\right\} & =\frac{1}{2 m_{n}} \operatorname{Tr}\left\{\left[\hat{V}_{n, n-1}, \hat{p}_{n}^{2}\right] \hat{\rho}_{\xi}\left(t_{f}\right)\right\}+\frac{1}{2 m_{i}} \operatorname{Tr}\left\{\left[\hat{V}_{n, n+1}, \hat{p}_{n}^{2}\right] \hat{\rho}_{\xi}\left(t_{f}\right)\right\} .
\end{aligned}
$$

Substituting Eqs. (19-21) into Eq. (17) yields

$$
\frac{1}{\mathcal{Z}} \operatorname{Tr}\left\{\dot{\hat{\rho}}_{\xi}\left(t_{f}\right) \hat{H}_{n}\right\}=P_{\xi_{n}}\left(t_{f}, \boldsymbol{\xi}\right]+P_{\gamma_{n}}\left(t_{f}, \boldsymbol{\xi}\right]+P_{n-1 \rightarrow n}\left(t_{f}, \boldsymbol{\xi}\right]+P_{n+1 \rightarrow n}\left(t_{f}, \boldsymbol{\xi}\right]
$$

where $\mathcal{Z} \equiv \operatorname{Tr}\left\{\hat{\rho}\left(t_{f}\right)\right\}$. We can identify

$$
P_{\xi_{n}}\left(t_{f}, \boldsymbol{\xi}\right] \equiv \mathcal{Z}^{-1} \frac{\xi_{n}\left(t_{f}\right)}{m_{n}} \operatorname{Tr}\left\{\hat{p}_{n} \hat{\rho}_{\xi}\left(t_{f}\right)\right\}
$$

as the energy flow into the oscillator $n$ from its private bath and

$$
P_{\gamma_{n}}\left(t_{f}, \boldsymbol{\xi}\right] \equiv-\mathcal{Z}^{-1} \frac{2 \gamma_{n}}{m_{n}} \operatorname{Tr}\left\{\hat{p}_{n}^{2} \hat{\rho}_{\xi}\left(t_{f}\right)\right\}
$$

the rate of the energy dissipated back to the bath. We note that Eqs. $(23,24)$ coincides with the definition of the oscillator-bath heat exchange in [17] through the semiclassical Langevin equation. The remaining two expressions in (22) describe the energy exchange between the neighboring oscillators

$$
\begin{aligned}
& P_{n-1 \rightarrow n}\left(t_{f}, \boldsymbol{\xi}\right] \equiv-\mathcal{Z}^{-1} \frac{i}{2 m_{n}} \operatorname{Tr}\left\{\left[\hat{p}_{n}^{2}, \hat{V}_{n, n-1}\right] \hat{\rho}_{\xi}\left(t_{f}\right)\right\}, \\
& P_{n+1 \rightarrow n}\left(t_{f}, \boldsymbol{\xi}\right] \equiv-\mathcal{Z}^{-1} \frac{i}{2 m_{n}} \operatorname{Tr}\left\{\left[\hat{p}_{n}^{2}, \hat{V}_{n, n+1}\right] \hat{\rho}_{\xi}\left(t_{f}\right)\right\} .
\end{aligned}
$$

Since $\operatorname{Tr}\left\{\dot{\hat{\rho}}_{\xi}\left(t_{f}\right) \hat{H}_{n}\right\} / \mathcal{Z}$ is the change of the trajectory-wise internal energy of oscillator $n$, we then interpret $P_{n-1 \rightarrow n}\left(t_{f}, \boldsymbol{\xi}\right]$ as the power delivered to oscillator $n$ by oscillator $n-1$ to it, while $P_{n+1 \rightarrow n}\left(t_{f}, \xi\right]$ as the power by oscillator $n+1$. The mutual coupling between the oscillators consists of various components as shown in (4), it proves convenient to further decompose the energy exchange between the oscillators into the sum of the contributions from these components

$$
P_{v \rightarrow n}\left(t_{f}, \boldsymbol{\xi}\right]=\sum_{\eta=2}^{4} P_{v \rightarrow n}^{(\eta)}\left(t_{f}, \boldsymbol{\xi}\right]
$$

where $v=n \pm 1$ and the contribution from each components is defined by

$$
P_{\nu \rightarrow n}^{(\eta)}\left(t_{f}, \boldsymbol{\xi}\right] \equiv-\mathcal{Z}^{-1} \frac{\lambda_{\eta}}{\eta} \frac{i}{2 m_{n}} \operatorname{Tr}\left\{\left[\hat{p}_{n}^{2},\left(\hat{\chi}_{n}-\hat{\chi}_{v}\right)^{\eta}\right] \hat{\rho}_{\xi}\left(t_{f}\right)\right\} .
$$

At this moment, for each trajectory $\xi$, we have denoted the thermodynamic quantities $O$ by the expectation value $O\left(t_{f}, \xi\right]$, which could be, e.g., the heat exchange between the bath and the primary system, heat flow inside the primary system, local kinetic and potential energy, etc. Their nonequilibrium dynamics can be investigated once we get hold of the stochastic density matrix $\hat{\rho}_{\xi}(t)$ at any moment $t$. However, $O\left(t_{f}, \xi\right]$ will not correspond to the observable we measure. It is too fine-grained, corresponding to each realization of the stochastic noise $\xi$, and we still 
need to perform the appropriate ensemble average. For example, in Eqs. (23), (24), and (26), we have used the first and the second moments of the momentum operator at the $n^{\text {th }}$ site

$$
p_{n}\left(t_{f}, \boldsymbol{\xi}\right]=\operatorname{Tr}\left\{\hat{p}_{n} \hat{\rho}_{\xi}\left(t_{f}\right)\right\}, \quad p_{n}^{2}\left(t_{f}, \boldsymbol{\xi}\right]=\operatorname{Tr}\left\{\hat{p}_{n}^{2} \hat{\rho}_{\xi}\left(t_{f}\right)\right\},
$$

and the quantity

$$
\left.w_{v \rightarrow n}^{(\eta)}\left(t_{f}, \boldsymbol{\xi}\right]=-\frac{i}{2 \eta} \operatorname{Tr}\left\{\left[\hat{p}_{n}^{2},\left(\hat{\chi}_{n}-\hat{\chi}_{v}\right)^{\eta}\right)\right] \hat{\rho}_{\xi}\left(t_{f}\right)\right\} .
$$

Thus after performing the ensemble average introduced in (8), we can write the averaged heat current respectively associated with (23), (24), and (26) as

$$
\begin{aligned}
P_{\xi_{n}}\left(t_{f}\right) & =\mathcal{Z}^{-1} \frac{1}{m_{n}}\left\langle\xi_{n}\left(t_{f}\right) p_{n}\left(t_{f}, \boldsymbol{\xi}\right]\right\rangle, \\
P_{\gamma_{n}}\left(t_{f}\right) & =-\mathcal{Z}^{-1} \frac{2 \gamma_{n}}{m_{n}}\left\langle p_{n}^{2}\left(t_{f}, \boldsymbol{\xi}\right]\right\rangle, \\
P_{\nu \rightarrow n}^{(\eta)}\left(t_{f}\right) & =\mathcal{Z}^{-1} \frac{\lambda_{\eta}}{m_{n}}\left\langle\left\langle w_{v \rightarrow n}^{(\eta)}\left(t_{f}, \boldsymbol{\xi}\right]\right\rangle .\right.
\end{aligned}
$$

Now with the functional method introduced in Appendix B, the results we obtained earlier can be concisely expressed as the functional derivatives of a generating functional

$$
\begin{aligned}
p_{n}\left(t_{f}, \boldsymbol{\xi}\right] & =-\left.i \frac{\partial}{\partial h_{n}} \mathcal{Z}_{\xi}\left[\boldsymbol{j}, \boldsymbol{j}^{\prime}, \boldsymbol{h}\right)\right|_{j=j^{\prime}=\boldsymbol{h}=0} \\
p_{n}^{2}\left(t_{f}, \boldsymbol{\xi}\right] & =-\left.\frac{\partial^{2}}{\partial h_{n}^{2}} \mathcal{Z}_{\xi}\left[\boldsymbol{j}, \boldsymbol{j}^{\prime}, \boldsymbol{h}\right)\right|_{j=j^{\prime}=\boldsymbol{h}=0} \\
w_{\nu \rightarrow n}^{(\eta)}\left(t_{f}, \boldsymbol{\xi}\right] & =-\left.(-i)^{\eta} \frac{\partial}{\partial h_{n}}\left[\frac{\delta}{\delta j_{n}\left(t_{f}\right)}-\frac{\delta}{\delta j_{\nu}\left(t_{f}\right)}\right]^{\eta-1} \mathcal{Z}_{\xi}\left[\boldsymbol{j}, \boldsymbol{j}^{\prime}, \boldsymbol{h}\right)\right|_{\boldsymbol{j}=j^{\prime}=\boldsymbol{h}=0},
\end{aligned}
$$

and in particular, $\operatorname{Tr}\left\{\rho\left(t_{f}\right)\right\}=\left\langle\left\langle\mathcal{Z}_{\xi}[0]\right\rangle\right.$, where the generating functional $\mathcal{Z}_{\xi}\left[\boldsymbol{j}, \boldsymbol{j}^{\prime}, \boldsymbol{h}\right)$ of central importance is defined by

$$
\mathcal{Z}_{\xi}\left[\boldsymbol{j}, \boldsymbol{j}^{\prime}, \boldsymbol{h}\right)=\int d \boldsymbol{r}_{i} d \boldsymbol{q}_{i} \int d \boldsymbol{r}_{f} \int_{\boldsymbol{r}(0)=\boldsymbol{r}_{i}}^{\boldsymbol{r}\left(t_{f}\right)=\boldsymbol{r}_{f}} \mathcal{D} \boldsymbol{r} \int_{\boldsymbol{q}(0)=\boldsymbol{q}_{i}}^{\boldsymbol{q}\left(t_{f}\right)=\boldsymbol{h}} \mathcal{D} \boldsymbol{q} e^{i \mathcal{S}_{\xi}\left[\boldsymbol{r}, \boldsymbol{q} ; \boldsymbol{j}, j^{\prime}\right]} \varrho\left(\boldsymbol{r}_{i}, \boldsymbol{q}_{i}\right),
$$

with the action given by

$$
\mathcal{S}_{\xi}\left[\boldsymbol{r}, \boldsymbol{q} ; \boldsymbol{j}, \boldsymbol{j}^{\prime}\right]=\mathcal{S}_{\xi}[\boldsymbol{r}, \boldsymbol{q}]+\int_{0}^{t_{f}} d s\left\{\boldsymbol{j}(s) \cdot \boldsymbol{r}(s)+\boldsymbol{j}^{\prime}(s) \cdot \boldsymbol{q}(s)\right\} .
$$

We have introduced the shorthand notation $\mathcal{Z}_{\xi}[0]$ for $\mathcal{Z}_{\xi}\left[\boldsymbol{j}=0, \boldsymbol{j}^{\prime}=0, \boldsymbol{h}=0\right.$ ). Two external, nondynamical currents $\boldsymbol{j}, \boldsymbol{j}^{\prime}$ and one external parameter $\boldsymbol{h}$ are introduced in the context of the in-in formalism to facilitate the functional method.

As of now, we have kept the discussions very general: (a) We allow general nonlinearities in the chain. Nonetheless, when nonlinearities are presented, the generating functional cannot be evaluated exactly and the perturbative treatment is needed, and (b) We allow each oscillator linearly coupled their own private linear bath, but do not restrict the coupling strength. In what follows, we shall carry out the detailed calculations specified by Eqs. (31-36) and address the existence of the NESS across a nonlinear chain made of two nonlinear oscillators, which are put in contact with their private thermal baths.

\section{The zeroth-order of the heat exchange between an oscillator and their private baths}

With the functional perturbative approach sketched in Sec. 2, we are in a position to compute the heat flow across the nonlinear chain in the weak nonlinearity regime. For the sake of simplicity, we will demonstrate in detail only for the case of two anharmonic oscillators. The argument generalizes to $N>2$ anharmonic oscillators. We outline the steps as follows: 
1. We show in Appendix $C$ that thanks to the natures of the linear dynamics at late time, $t_{f} \rightarrow \infty$, the stochastic generating functional $\mathcal{Z}_{0 \xi}\left[\boldsymbol{j}, \boldsymbol{j}^{\prime}, \boldsymbol{h}\right)$ for the linear chain reduces a simple form [see Eqs. (45-48)]. These can be straightforwardly generalized to the case of $N>2$ linear harmonic oscillators in the chain. One only needs to replace the causal propagator corresponding to two-oscillator and two-bath configuration with the one under investigation.

2. With the help this simplified stochastic generating functional and the Wick contraction, we compute the perturbative corrections for the quantities of our interest at the late time to the first order in the nonlinear coupling constants.

3. We show that the contribution from the initial state of the chain at the late time $t_{f} \rightarrow \infty$ vanishes due to the damping effect. Furthermore, the internal energy of each oscillator is stationary and therefore NESS may be argued, at least up to the first order in nonlinear coupling constants.

We have already seen in Eqs. (35-36) that the generating functional Eq. (37) is of crucial importance to obtain various thermodynamic quantities. To illustrate the stochastic functional perturbative approach, we consider three types of nonlinearities: $\alpha$-FPUT, $\beta$-FPUT and KG types of anharmonicity in the chain, consisting of only two oscillators. We assume the oscillator-bath coupling constants are equal $e_{1}=e_{2}=e$ and therefore the retarded Green's functions of the two private baths take the same form, i.e., $G_{R}^{(1)}=G_{R}^{(2)}$. Furthermore, for the sake of simplicity, we assume uniform mass distribution across the chain, so we set mass of each oscillator to unity without loss of generality. We can always recover the mass dependence in the relevant energy fluxes through dimensional consideration or redefining parameters and displacements in the Lagrangian.

In the context of the perturbative treatment, one splits the chain Lagrangian into two parts: $L_{\chi}=L_{0}+L_{\mathrm{NL}}$, where $L_{0}$ describes the linearly coupled oscillators

$$
L_{0}[\chi]=\frac{1}{2} \sum_{n=1}^{2}\left[\dot{\chi}_{n}^{2}-\omega_{0}^{2} \chi_{n}^{2}\right]-\frac{\lambda_{2}}{2}\left(\chi_{1}-\chi_{2}\right)^{2},
$$

and $L_{\mathrm{NL}}$ accounts for the nonlinear self- and intra-oscillator couplings,

$$
L_{\mathrm{NL}}[\chi]=-\left[\frac{\lambda_{3}}{3}\left(\chi_{1}-\chi_{2}\right)^{3}+\frac{\lambda_{4}}{4}\left(\chi_{1}-\chi_{2}\right)^{4}+\frac{\lambda_{\mathrm{KG}}}{4}\left(\chi_{1}^{4}+\chi_{2}^{4}\right)\right] .
$$

In (40), the first two terms on the righthand side describe nonlinear intra-oscillator couplings: the cubic term for the $\alpha$-FPUT coupling and the quartic term for the $\beta$-FPUT coupling. The last term is the KG quartic on-site pin potential.

To the first order of the coupling constants, the stochastic propagating function Eq. (A.11) in this case generically takes the form

$$
\begin{array}{rl}
\mathcal{J}_{\xi}\left(\boldsymbol{r}_{f}, \boldsymbol{h}, t_{f} ; \boldsymbol{r}_{i}, \boldsymbol{q}_{i} ; \boldsymbol{j}, \boldsymbol{j}^{\prime}\right)=\int_{\boldsymbol{r}(0)=\boldsymbol{r}_{i}}^{\boldsymbol{r}\left(t_{f}\right)=\boldsymbol{r}_{f}} & \mathcal{D} \boldsymbol{r} \int_{\boldsymbol{q}(0)=\boldsymbol{q}_{i}}^{\boldsymbol{q}\left(t_{f}\right)=\boldsymbol{h}} \mathcal{D} \boldsymbol{q} \exp \left\{i \mathcal{S}_{\xi}^{(0)}\left[\boldsymbol{r}, \boldsymbol{q} ; \boldsymbol{j}, \boldsymbol{j}^{\prime}\right]\right\} \\
& \times\left\{1+i \sum_{k l m r} \int_{0}^{t_{f}} d s\left[\sigma_{k l m r} r_{k}(s) q_{l}(s) q_{m}(s) q_{r}(s)+\mu_{k l m r} r_{k}(s) r_{l}(s) r_{m}(s) q_{r}(s)\right]\right. \\
& \left.+\sum_{k l m} \int_{0}^{t_{f}} d s\left[\sigma_{k l m} r_{k}(s) q_{l}(s) q_{m}(s)+\mu_{k l m} r_{k}(s) r_{l}(s) q_{m}(s)\right]\right\}
\end{array}
$$

where the nonlinear coupling constants $\lambda_{\mathrm{KG}}, \lambda_{4}$ and $\lambda_{3}$ have been absorbed into $\sigma_{k l m r}, \mu_{k l m r}$ and $\sigma_{k l m}$ and $\mu_{k l m}$ respectively. Thus the stochastic generating functional Eq. (37) is then given by

$$
\mathcal{Z}_{\xi}\left[\boldsymbol{j}, \boldsymbol{j}^{\prime}, \boldsymbol{h}\right)=\int d \boldsymbol{r}_{i} d \boldsymbol{q}_{i} \int d \boldsymbol{r}_{f} \mathcal{J}_{\xi}\left(\boldsymbol{r}_{f}, \boldsymbol{h}, t_{f} ; \boldsymbol{r}_{i}, \boldsymbol{q}_{i} ; \boldsymbol{j}, \boldsymbol{j}^{\prime}\right) \varrho\left(\boldsymbol{r}_{i}, \boldsymbol{q}_{i}\right) .
$$

Since we will perform the perturbative calculations based on the zeroth-order results, we will denote $\left\langle\langle\bullet\rangle_{0}\right.$ as the ensemble average with respect to the zeroth-order stochastic generating functional $Z_{\xi}^{(0)}$, that is, the linear chain, which takes the same form as (42) with $\mathcal{J}_{\xi}$ replaced by $\mathcal{J}_{\xi}^{(0)}$. The latter does not contain any nonlinear contribution in (41). 
The stochastic action $\mathcal{S}_{\xi}^{(0)}$ for the linear chain is

$$
\begin{array}{r}
\mathcal{S}_{\xi}^{(0)}\left[\boldsymbol{r}, \boldsymbol{q}, \boldsymbol{j}, \boldsymbol{j}^{\prime}\right]=\int_{0}^{t_{f}} d s\left\{\dot{\boldsymbol{q}}^{T}(s) \cdot \dot{\boldsymbol{r}}(s)-\boldsymbol{q}^{T}(s) \cdot \boldsymbol{\Omega}^{2} \cdot \boldsymbol{r}(s)+\boldsymbol{q}^{T}(s) \cdot \boldsymbol{j}^{\prime}(s)+\boldsymbol{r}^{T} \cdot \boldsymbol{j}(s)\right. \\
\left.+\boldsymbol{q}^{T}(s) \cdot \boldsymbol{\xi}(s)+\int_{0}^{t} d s^{\prime} \boldsymbol{q}^{T}(s) \boldsymbol{G}_{R}\left(s, s^{\prime}\right) \boldsymbol{r}\left(s^{\prime}\right)\right\}
\end{array}
$$

where $\sqrt{\omega_{0}^{2}+\lambda_{2}}$ is understood as the bare frequency of the linear oscillator, and $\lambda_{2}$ is the mutual coupling between two oscillators,

$$
\mathbf{\Omega}^{2} \equiv\left[\begin{array}{cc}
\omega_{0}^{2}+\lambda_{2} & -\lambda_{2} \\
-\lambda_{2} & \omega_{0}^{2}+\lambda_{2}
\end{array}\right] .
$$

This action essentially describes the dynamics of the coupled, dissipative oscillators, driven by the quantum fluctuations of their individual baths [17].

In Eq. (Appendix C), we find in the limit $t_{f} \rightarrow \infty$, the stochastic generating functional $\mathcal{Z}_{\xi}^{(0)}$ can be factored into

$$
\mathcal{Z}_{\xi}^{(0)}\left[\boldsymbol{j}, \boldsymbol{j}^{\prime}, \boldsymbol{h}\right)=\mathscr{Z}^{(0)}\left[\varrho_{i}, \boldsymbol{j}\right] \exp \left\{i \overline{\mathscr{S}}_{\xi}^{(0)}[\boldsymbol{j}, \boldsymbol{h}]+i \bar{\Xi}^{(0)}\left[\boldsymbol{j}, \boldsymbol{j}^{\prime}, \boldsymbol{h}\right)\right\},
$$

according to their dependence on the initial state and the external sources, where

$$
\begin{aligned}
\mathscr{Z}^{(0)}\left[\varrho_{i}, \boldsymbol{j}\right] & =\int d \boldsymbol{r}_{i} \exp \left\{i \int_{0}^{t_{f}} d s \boldsymbol{j}^{T}(s) \cdot \boldsymbol{D}_{1}(s) \cdot \boldsymbol{r}_{i}\right\} \varrho\left(\boldsymbol{r}_{i}, \int_{0}^{t_{f}} d s \boldsymbol{D}_{2}(s) \cdot \boldsymbol{j}(s)\right), \\
\overline{\mathscr{S}}_{\xi}^{(0)}[\boldsymbol{j}, \boldsymbol{h}) & =\boldsymbol{h}^{T} \cdot \int_{0}^{t_{f}} d s \dot{\boldsymbol{D}}_{2}\left(t_{f}-s\right) \cdot \boldsymbol{\xi}(s)+\int_{0}^{t_{f}} d s \int_{0}^{t_{f}} d s^{\prime} \boldsymbol{j}^{T}(s) \cdot \mathfrak{D}\left(s-s^{\prime}\right) \cdot \boldsymbol{\xi}\left(s^{\prime}\right), \\
\overline{\mathfrak{S}}^{(0)}\left[\boldsymbol{j}, \boldsymbol{j}^{\prime}, \boldsymbol{h}\right] & =\int_{0}^{t_{f}} d s \int_{0}^{t_{f}} d s^{\prime} \boldsymbol{j}^{T}(s) \cdot \mathfrak{D}\left(s-s^{\prime}\right) \cdot \boldsymbol{j}^{\prime}\left(s^{\prime}\right)+\boldsymbol{h}^{T} \cdot \int_{0}^{t_{f}} d s \dot{\boldsymbol{D}}_{2}\left(t_{f}-s\right) \cdot \boldsymbol{j}^{\prime}(s) .
\end{aligned}
$$

The matrices $\boldsymbol{D}_{i}(s)$ are the homogenous solutions to Eq. (C.5), with the initial conditions $\boldsymbol{D}_{1}(0)=\mathbb{I}, \dot{\boldsymbol{D}}_{1}(0)=0$, $\boldsymbol{D}_{2}(0)=0$ and $\dot{\boldsymbol{D}}_{2}(0)=\mathbb{I}$, and $\mathfrak{D}(s)=\Theta(s) \boldsymbol{D}_{2}(s)$ is the causal (retarded) Green's function of the chain. In the case of two oscillators, the Laplace transforms of $\boldsymbol{D}_{i}(s)$ are given by Eqs. (C.11) and (C.12).

The first factor on the right hand side of Eq. (45) contains the contributions due to the initial state of the coupled oscillators. One would naturally ask whether the NESS thermodynamic quantities and functional derivatives generated by Eq. (45) depends on the initial state? For linear chain or the zeroth-order of nonlinear chain, all the heat fluxes are related to functional derivatives $\delta / \delta \boldsymbol{j}\left(t_{f}\right)$ [see Eqs. (34-36)]. According to Theorem 5 in Appendix D, in the late time limit, the NESS heat fluxes for the linear chain or at the zeroth-order for nonlinear chain do not depend on the initial state of the chain. For an anharmonic chain with the $\alpha, \beta$-FPUT and KG types of nonlinearities, we will show in Sec. 4, by the virtues of theorems in Appendix D, at least to the first-order of the nonlinear coupling, the NESS exists, and is independent of the initial states.

We conjecture that this conclusion holds to arbitrary order of the nonlinear coupling constants for $\alpha$, $\beta$-FPUT and KG types of nonlinearities, as long as the coupling is weak such that our perturbation calculation is still valid. The reason is the following: Physically, it is plausible that because of the damping, the initial information about the chain will finally decay for an arbitrary order of the nonlinear coupling constants. Mathematically, this implies that contribution of the steady-state heat flow from the functional derivation of the partition function $\mathscr{Z}^{(0)}\left[\varrho_{i}, j\right][$ see Eq. (45)] will eventually vanish, and requires that the contribution from each higher order is smaller than that from previous order to ensure the validity of the perturbative treatment. The observation that the initial information is irrelevant in the NESS effectively amounts to setting $\mathscr{Z}^{(0)}\left[\varrho_{i}, \boldsymbol{j}=0\right]=1$ from the very beginning of the computation of the steady-state heat exchange. That is to say, we can work with the new stochastic generating functional $\check{\mathcal{Z}}_{\xi}^{(0)}$,

$$
\check{\mathcal{Z}}_{\xi}^{(0)}\left[\boldsymbol{j}, \boldsymbol{j}^{\prime}, \boldsymbol{h}\right)=\exp \left\{i \overline{\mathscr{S}}_{\xi}^{(0)}[\boldsymbol{j}, \boldsymbol{h})+i \bar{\Xi}^{(0)}\left[\boldsymbol{j}, \boldsymbol{j}^{\prime}, \boldsymbol{h}\right)\right\},
$$

where the check mark indicates the initial state contribution is ignored. It is then straightforward to compute the high order correction of the steady-state heat flows across the chain. We speculate the heat flow should satisfy their respective heat balance order by order for a chain with either one of $\alpha, \beta$-FPUT and KG types of nonlinearities. 
Next we first apply the approach we have developed so far to the zeroth-order contributions in the quantities defined in Eqs. (34)-(36), which corresponds to the heat exchange across the linear chain. In this case the generating functional only contains one single term: $\exp \left\{i \boldsymbol{h}^{T} \cdot \int_{0}^{t_{f}} d s \dot{\boldsymbol{D}}_{2}\left(t_{f}-s\right) \cdot \boldsymbol{\xi}(s)\right\}$. Thus we find that for the $n^{\text {th }}$ oscillator, its first moment of momentum at the zeroth order is

$$
p_{n}^{(0)}\left(t_{f}, \boldsymbol{\xi}\right]=-\left.i \frac{\partial}{\partial h_{n}} \mathcal{Z}_{\xi}^{(0)}\left[\boldsymbol{j}, \boldsymbol{j}^{\prime}, \boldsymbol{h}\right)\right|_{\boldsymbol{j}=\boldsymbol{j}^{\prime}=\boldsymbol{h}=0}=\int_{0}^{t_{f}} d s\left[\dot{\boldsymbol{D}}_{2}\left(t_{f}-s\right) \cdot \boldsymbol{\xi}(s)\right]_{n},
$$

so that the energy flow pumped in by its private bath is

$$
\begin{aligned}
P_{\xi_{n}}^{(0)}\left(t_{f}\right) & =\sum_{m=1}^{2} \int_{0}^{t_{f}} d s\left[\dot{\boldsymbol{D}}_{2}\left(t_{f}-s\right)\right]_{n m}\left[\boldsymbol{G}_{H}\left(t_{f}-s\right)\right]_{m n} \\
& =\int_{0}^{t_{f}} d s\left[\dot{\boldsymbol{D}}_{2}(s) \cdot \boldsymbol{G}_{H}(s)\right]_{n n}=\int_{-\infty}^{t_{f}} d s\left[\dot{\boldsymbol{D}}_{2}(s) \cdot \boldsymbol{G}_{H}(s)\right]_{n n},
\end{aligned}
$$

according to (31) and (34). Similarly, one can show the second moment of the momentum is given by

$$
\begin{aligned}
p_{n}^{2(0)}\left(t_{f}, \boldsymbol{\xi}\right] & =-\left.\frac{\partial^{2} Z_{0 \xi}\left[\boldsymbol{j}=0, \boldsymbol{j}^{\prime}=0, \boldsymbol{h}\right)}{\partial h_{n}^{2}}\right|_{\boldsymbol{h}=0} \\
& =\int_{0}^{t_{f}} d s \int_{0}^{t_{f}} d s^{\prime}\left[\dot{\boldsymbol{D}}_{2}(s) \boldsymbol{G}_{H}\left(s^{\prime}-s\right) \dot{\boldsymbol{D}}_{2}\left(s^{\prime}\right)\right]_{n n} \\
& =\int_{-\infty}^{t_{f}} d s \int_{-\infty}^{t_{f}} d s^{\prime}\left[\dot{\mathfrak{D}}(s) \cdot \boldsymbol{G}_{H}\left(s^{\prime}-s\right) \cdot \dot{\mathfrak{D}}_{2}\left(s^{\prime}\right)\right]_{n n}
\end{aligned}
$$

The energy flow dissipated back into the thermal bath is

$$
P_{\gamma_{n}}^{(0)}\left(t_{f}\right)=-2 \gamma \int_{-\infty}^{t_{f}} d s \int_{-\infty}^{t_{f}} d s^{\prime}\left[\dot{\mathfrak{D}}(s) \cdot \boldsymbol{G}_{H}\left(s^{\prime}-s\right) \cdot \dot{\mathfrak{D}}_{2}\left(s^{\prime}\right)\right]_{n n} .
$$

For the energy flow between two oscillators at the zeroth order, we only need to consider the $w_{v \rightarrow n}^{(0,2)}\left(t_{f}, \boldsymbol{\xi}\right]$ term,

$$
w_{v \rightarrow n}^{(0,2)}\left(t_{f}, \boldsymbol{\xi}\right]=\left.\frac{\partial}{\partial h_{n}}\left[\frac{\delta}{\delta j_{n}\left(t_{f}\right)}-\frac{\delta}{\delta j_{v}\left(t_{f}\right)}\right] \mathcal{Z}_{\xi}^{(0)}\left[\boldsymbol{j}, \boldsymbol{j}^{\prime}, \boldsymbol{h}\right)\right|_{\boldsymbol{j}=j^{\prime}=\boldsymbol{h}=0} .
$$

Here, we note that notational difference: From Eq. (30), the first superscript $a$ in $w_{v \rightarrow n}^{(a, \eta)}\left(t_{f}\right.$, $\xi$ ] denotes the perturbative order of the nonlinear coupling constants, $\lambda_{3}, \lambda_{4}, \lambda_{\mathrm{KG}}$, as one will see in next section. From Eqs. (45-48), we observe that only $\mathscr{Z}^{(0)}\left[\varrho_{i}, \boldsymbol{j}\right]$ and $\exp \left\{i \overline{\mathscr{S}}_{\xi}^{(0)}[\boldsymbol{j}, \boldsymbol{h})\right\}$ in the generating functional contribute. Moreover, from the discussions in Theorem 5, in the late time limit, the only non-vanishing results in $w_{v \rightarrow n}^{(0,2)}$ come from acting the functional derivatives $\delta / \delta j_{n}\left(t_{f}\right), \delta / \delta j_{v}\left(t_{f}\right)$ of $\overline{\mathscr{S}}_{0 \xi}[\boldsymbol{j}, \boldsymbol{h})$. Therefore, we obtain

$$
\begin{aligned}
w_{v \rightarrow n}^{(0,2)}\left(t_{f}, \boldsymbol{\xi}\right] & =-\int_{0}^{t_{f}} d s\left[\dot{\boldsymbol{D}}_{2}\left(t_{f}-s\right) \boldsymbol{\xi}(s)\right]_{n} \int_{0}^{t_{f}} d s^{\prime}\left(\left[\mathfrak{D}\left(t_{f}-s^{\prime}\right) \boldsymbol{\xi}\left(s^{\prime}\right)\right]_{n}-\left[\mathfrak{D}\left(t_{f}-s^{\prime}\right) \boldsymbol{\xi}\left(s^{\prime}\right)\right]_{v}\right) \\
& \left.=-\sum_{k l} \int_{0}^{t_{f}} d s \int_{0}^{t_{f}} d s^{\prime}\left[\dot{\boldsymbol{D}}_{2}\left(t_{f}-s\right)\right]_{n k}[\boldsymbol{\xi}(s)]_{k}\left(\left[\mathfrak{D}\left(t_{f}-s^{\prime}\right)\right]_{n l}\left[\boldsymbol{\xi}\left(s^{\prime}\right)\right]_{l}-\left[\mathfrak{D}\left(t_{f}-s^{\prime}\right)\right]_{v l} \boldsymbol{\xi}\left(s^{\prime}\right)\right]_{l}\right)
\end{aligned}
$$

Using Eq. (10), we find

$$
\begin{aligned}
w_{v \rightarrow n}^{(0,2)}\left(t_{f}\right) & =-\int_{0}^{t_{f}} d s \int_{0}^{t_{f}} d s^{\prime}\left(\left[\dot{\boldsymbol{D}}_{2}(s) \boldsymbol{G}_{H}\left(s^{\prime}-s\right) \mathfrak{D}\left(s^{\prime}\right)\right]_{n n}-\left[\dot{\boldsymbol{D}}_{2}(s) \boldsymbol{G}_{H}\left(s^{\prime}-s\right) \mathfrak{D}\left(s^{\prime}\right)\right]_{n v}\right) \\
& =-\int_{-\infty}^{t_{f}} d s \int_{-\infty}^{t_{f}} d s^{\prime}\left(\left[\dot{\mathfrak{D}}(s) \boldsymbol{G}_{H}\left(s^{\prime}-s\right) \mathfrak{D}\left(s^{\prime}\right)\right]_{n n}-\left[\dot{\mathfrak{D}}(s) \boldsymbol{G}_{H}\left(s^{\prime}-s\right) \mathfrak{D}\left(s^{\prime}\right)\right]_{n v}\right)
\end{aligned}
$$

It can be straightforwardly shown that for $t_{f} \gg \gamma^{-1}$, Eqs. (51, 52 56) approach time-independent constants because of damping. The diagrammatic representation of Eqs. $(51,5256)$ is given in Fig. 2. Further illustrations of these diagrams will be discussed in Sec. 5 

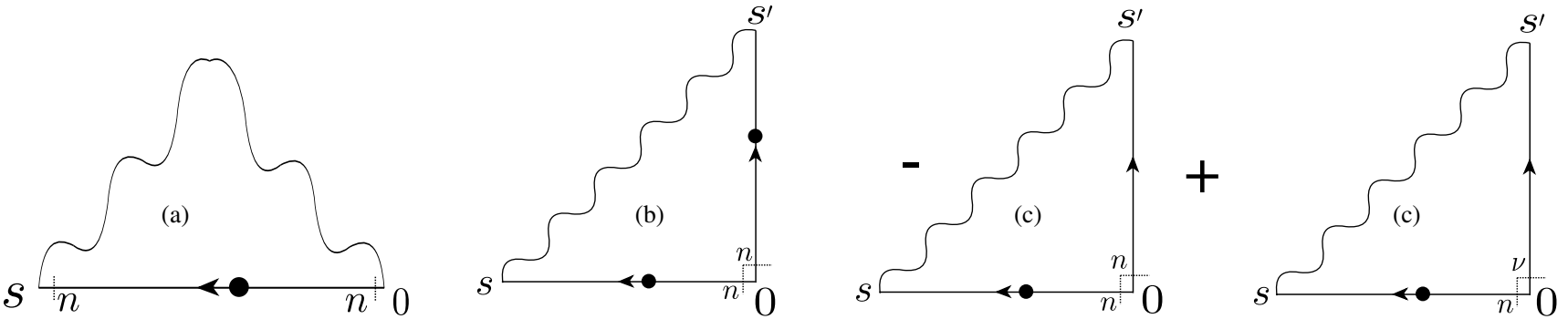

Figure 2: Time-domain diagrammatic representation of the zeroth-order energy flux including (a) $P_{\xi_{n}}$ (b) $p_{n}^{2(0)}$ (c) $w_{v \rightarrow n}^{(0,2)}$. As we show Sec. 5.1, the first digram in (c) actually vanishes in the late time limit.

\section{The first-order corrections}

From (41), we observe that for the first-order corrections, the three types of nonlinearities contribute independently to the first-order corrections, so in what follows, we will first discuss the contributions from the $\beta$-FPUT and KG nonlinearities, and then the $\alpha$-FPUT nonlinearity. In the end, we put them together to form the overall first-order corrections, and examine the effects of these nonlinearities on the heat transport in NESS.

\subsection{Stochastic generating functional $\mathcal{Z}_{\xi}^{(1)}(0)$}

We start with the the first-order correction to the stochastic generating functional. From Eq. (41), we can easily show that

$$
\begin{aligned}
\mathcal{Z}_{\xi}^{(1)}[0]=i \int_{0}^{t_{f}} d s & \left.\sum_{k l m r}\left[\sigma_{k l m r}\left\langle r_{k}(s) q_{l}(s) q_{m}(s) q_{r}(s)\right\rangle_{0}+\mu_{k l m r}\left\langle r_{k}(s) r_{l}(s) r_{m}(s) q_{r}(s)\right\rangle_{0}\right]\right|_{j=j^{\prime}=\boldsymbol{h}=0} \\
& +\left.i \int_{0}^{t_{f}} d s \sum_{k l m}\left[\sigma_{k l m}\left\langle r_{k}(s) q_{l}(s) q_{m}(s)\right\rangle_{0}+\mu_{k l m}\left\langle r_{k}(s) r_{l}(s) q_{m}(s)\right\rangle_{0}\right]\right|_{j=j^{\prime}=\boldsymbol{h}=0}
\end{aligned}
$$

where

$$
\begin{aligned}
\left\langle r_{k}(s) q_{l}(s) q_{m}(s)\right\rangle_{0} & =i \frac{\delta^{3} \mathcal{Z}_{\xi}^{(0)}\left[\boldsymbol{j}, \boldsymbol{j}^{\prime}, \boldsymbol{h}\right)}{\delta j_{k}(s) \delta j_{l}^{\prime}(s) \delta j_{m}^{\prime}(s)}, & \left.\left\langle r_{k}(s) q_{l}(s) q_{m}(s) q_{r}(s)\right\rangle_{0}\right\rangle_{0} & =\frac{\delta^{4} \mathcal{Z}_{\xi}^{(0)}\left[\boldsymbol{j}, \boldsymbol{j}^{\prime}, \boldsymbol{h}\right)}{\delta j_{k}(s) \delta j_{l}^{\prime}(s) \delta j_{m}^{\prime}(s) \delta j_{r}^{\prime}(s)}, \\
\left\langle r_{k}(s) r_{l}(s) q_{m}(s)\right\rangle_{0} & =i \frac{\delta^{3} \mathcal{Z}_{\xi}^{(0)}\left[\boldsymbol{j}, \boldsymbol{j}^{\prime}, \boldsymbol{h}\right)}{\delta j_{k}(s) \delta j_{l}(s) \delta j_{m}^{\prime}(s)}, & \left\langle r_{k}(s) r_{l}(s) r_{m}(s) q_{r}(s)\right\rangle_{0} & =\frac{\delta^{4} \boldsymbol{Z}_{\xi}^{(0)}\left[\boldsymbol{j}, \boldsymbol{j}^{\prime}, \boldsymbol{h}\right)}{\delta j_{k}(s) \delta j_{l}(s) \delta j_{m}(s) \delta j_{r}^{\prime}(s)}
\end{aligned}
$$

From Theorem 3 in Appendix D, the above four ensemble averages all vanish, so the generating functional $Z_{\xi}^{(1)}[0]$ does not have the first-order correction, that is, $\mathcal{Z}_{\xi}^{(1)}[0]=0$.

\subsection{Heat exchanges $P_{\xi_{n}}^{(1)}\left(t_{f}\right), P_{\gamma_{n}}^{(1)}\left(t_{f}\right)$ between the oscillator and its private bath}

Here we will compute the first-order correction to the rate of the energy exchange between the bath to the oscillator it belongs to.

\subsection{1. $K G$ and $\beta$-FPUT nonlinearities}

In (31) and (32), we have shown that the first-order corrections of the rates of the energy exchange $P_{\xi_{n}}^{(1)}\left(t_{f}\right), P_{\gamma_{n}}^{(1)}\left(t_{f}\right)$ between oscillator and its private bath, are proportional to $\left\langle\xi_{n}\left(t_{f}\right) p_{n}^{(1)}\left(t_{f}, \xi\right]\right\rangle$ and $\left\langle\left\langle p_{n}^{2(1)}\left(t_{f}, \boldsymbol{\xi}\right]\right\rangle\right.$, respectively. From Eqs. (34) and (35), the first-order corrections of $p_{n}\left(t_{f}, \xi\right]$ and $p_{n}^{2(1)}\left(t_{f}, \xi\right]$ are found to be

$$
p_{n}^{(1)}\left(t_{f}, \boldsymbol{\xi}\right]=\left.\int_{0}^{t_{f}} d s \sum_{k l m r}\left[\sigma_{k l m r} \partial_{n}\left\langle r_{k}(s) q_{l}(s) q_{m}(s) q_{r}(s)\right\rangle_{0}+\mu_{k l m r} \partial_{n}\left\langle r_{k}(s) r_{l}(s) r_{m}(s) q_{r}(s)\right\rangle_{0}\right]\right|_{j=j^{\prime}=\boldsymbol{h}=0},
$$




$$
p_{n}^{2(1)}\left(t_{f}, \boldsymbol{\xi}\right]=-\left.i \int_{0}^{t_{f}} d s \sum_{k l m r}\left[\sigma_{k l m r} \partial_{n}^{2}\left\langle r_{k}(s) q_{l}(s) q_{m}(s) q_{r}(s)\right\rangle_{0}+\mu_{k l m r} \partial_{n}^{2}\left\langle r_{k}(s) r_{l}(s) r_{m}(s) q_{r}(s)\right\rangle_{0}\right]\right|_{j=j^{\prime}=\boldsymbol{h}=0},
$$

where $\partial_{n}^{k} \equiv \partial^{k} / \partial h_{n}^{k}$ and

$$
\begin{aligned}
\left.\partial_{n}\left\langle r_{k}(s) q_{l}(s) q_{m}(s) q_{r}(s)\right\rangle_{0}\right|_{\boldsymbol{j}=\boldsymbol{j}^{\prime}=\boldsymbol{h}=0} & =\left.\frac{\partial \delta^{4} \mathcal{Z}_{0 \xi}\left[\boldsymbol{j}, \boldsymbol{j}^{\prime}, \boldsymbol{h}\right)}{\partial h_{n} \delta j_{k}(s) \delta j_{l}^{\prime}(s) \delta j_{m}^{\prime}(s) \delta j_{r}^{\prime}(s)}\right|_{\boldsymbol{j}=\boldsymbol{j}^{\prime}=\boldsymbol{h}=0} \\
\left.\partial_{n}\left\langle r_{k}(s) r_{l}(s) r_{m}(s) q_{r}(s)\right\rangle_{0}\right|_{\boldsymbol{j}=\boldsymbol{j}^{\prime}=\boldsymbol{h}=0} & =\left.\frac{\partial \delta^{4} \mathcal{Z}_{0 \xi}\left[\boldsymbol{j}, \boldsymbol{j}^{\prime}, \boldsymbol{h}\right)}{\partial h_{n} \delta j_{k}(s) \delta j_{l}(s) \delta j_{m}(s) \delta j_{r}^{\prime}(s)}\right|_{j=j^{\prime}=\boldsymbol{h}=0} \\
\left.\partial_{n}^{2}\left\langle r_{k}(s) q_{l}(s) q_{m}(s) q_{r}(s)\right\rangle_{0}\right|_{j=j^{\prime}=\boldsymbol{h}=0} & =\left.\frac{\partial \delta^{4} \mathcal{Z}_{0 \xi}\left[\boldsymbol{j}, \boldsymbol{j}^{\prime}, \boldsymbol{h}\right)}{\partial h_{n}^{2} \delta j_{k}(s) \delta j_{l}^{\prime}(s) \delta j_{m}^{\prime}(s) \delta j_{r}^{\prime}(s)}\right|_{j=j^{\prime}=\boldsymbol{h}=0} \\
\left.\partial_{n}^{2}\left\langle r_{k}(s) r_{l}(s) r_{m}(s) q_{r}(s)\right\rangle_{0}\right|_{\boldsymbol{j}=j^{\prime}=\boldsymbol{h}=0} & =\left.\frac{\partial^{2} \delta^{4} \mathcal{Z}_{0 \xi}\left[\boldsymbol{j}, \boldsymbol{j}^{\prime}, \boldsymbol{h}\right)}{\partial^{2} h_{n} \delta j_{k}(s) \delta j_{l}(s) \delta j_{m}(s) \delta j_{r}^{\prime}(s)}\right|_{\boldsymbol{j}=\boldsymbol{j}^{\prime}=\boldsymbol{h}=0}
\end{aligned}
$$

Not all of the terms in the integrand will contribute. From Theorem 3, one can easily see that Eqs. $(62,64)$ vanish. Hence, in order to find $\left\langle\left\langle\xi_{n}\left(t_{f}\right) p_{n}^{(1)}\left(t_{f}, \xi\right]\right\rangle\right\rangle$ and $\left\langle\left\langle p_{n}^{2(1)}\left(t_{f}, \xi\right]\right\rangle\right.$, we evaluate the following expressions

$$
\begin{aligned}
& \left.\Gamma_{k l m}^{n r}\left(t_{f}\right) \equiv \lim _{t_{f} \rightarrow \infty} \int_{0}^{t_{f}} d s\left\langle\left\langle\xi_{n}\left(t_{f}\right) \partial_{n}\left\langle r_{k}(s) r_{l}(s) r_{m}(s) q_{r}(s)\right\rangle_{0}\right\rangle\right\rangle\right|_{\boldsymbol{h}=0}, \\
& \tilde{\Gamma}_{k l m}^{n r}\left(t_{f}\right) \equiv-\left.i \lim _{t_{f} \rightarrow \infty} \int_{0}^{t_{f}} d s\left\langle\left\langle\partial_{n}^{2}\left\langle r_{k}(s) r_{l}(s) r_{m}(s) q_{r}(s)\right\rangle_{0}\right\rangle\right\rangle\right|_{\boldsymbol{h}=0} .
\end{aligned}
$$

Here to illustrate the implementation of the functional method, we will present the calculations in greater details.

Let us compute $\Gamma_{k l m}^{n r}\left(t_{f}\right)$ first. By Eqs. (45)-(48), it is straightforward to find

$$
\left.\frac{\partial \delta \mathcal{Z}_{\xi}^{(0)}\left[\boldsymbol{j}, \boldsymbol{j}^{\prime}, \boldsymbol{h}\right)}{\partial h_{n} \delta j_{r}^{\prime}(s)}\right|_{\dot{\boldsymbol{j}}^{\prime}=\boldsymbol{h}=0}=i\left[\dot{\boldsymbol{D}}_{2}\left(t_{f}-s\right)\right]_{n r} \mathscr{Z}^{(0)}\left[\varrho_{i}, \boldsymbol{j}\right] \exp \left\{i \overline{\mathscr{S}}_{\xi}^{(0)}[\boldsymbol{j}, \boldsymbol{h}=0)\right\}
$$

Thus Eq. (66) becomes

$$
\Gamma_{k l m}^{n r}\left(t_{f}\right)=\left.i \int_{0}^{t_{f}} d s\left\langle\left[\dot{\boldsymbol{D}}_{2}\left(t_{f}-s\right)\right]_{n r} \xi_{n}\left(t_{f}\right) \frac{\delta^{3} \mathscr{Z}^{(0)}\left[\varrho_{i}, \boldsymbol{j}\right] \exp \left\{i \overline{\mathscr{S}}_{\xi}^{(0)}[\boldsymbol{j}, \boldsymbol{h})\right\}_{k}}{\delta j_{k}(s) \delta j_{l}(s) \delta j_{m}(s)}\right\rangle\right|_{\boldsymbol{j}=\boldsymbol{h}=0}
$$

Theorem 7 in Appendix D shows that the only nonzero contribution in the late time limit $t_{f} \rightarrow \infty$ comes from the case when all three functional derivatives acts on $\exp \left\{i \overline{\mathscr{S}}_{\xi}^{(0)}[\boldsymbol{j}, \boldsymbol{h})\right\}$ only. Therefore, we arrive at

$$
\begin{gathered}
\Gamma_{k l m}^{n r}\left(t_{f}\right)=\sum_{p q j} \int \ldots \int_{0}^{t_{f}} d s d s_{1} d s_{2} d s_{3}\left\langle\left[\dot{\boldsymbol{D}}_{2}\left(t_{f}-s\right)\right]_{n r} \xi_{n}\left(t_{f}\right)\left[\mathfrak{D}\left(s-s_{1}\right)\right]_{k p} \xi_{p}\left(s_{1}\right)\left[\mathfrak{D}\left(s-s_{2}\right)\right]_{l q} \xi_{q}\left(s_{2}\right)\right. \\
\left.\times\left[\mathfrak{D}\left(s-s_{3}\right)\right]_{m j} \xi_{j}\left(s_{3}\right)\right\rangle d s d s_{1} d s_{2} d s_{3} .
\end{gathered}
$$

The Wick's theorem gives

$$
\begin{aligned}
\left\langle\left\langle\xi_{n}\left(t_{f}\right) \xi_{p}\left(s_{1}\right) \xi_{q}\left(s_{2}\right) \xi_{j}\left(s_{3}\right)\right\rangle\right\rangle & =\left[\boldsymbol{G}_{H}\left(t_{f}-s_{1}\right)\right]_{n p}\left[\boldsymbol{G}_{H}\left(s_{2}-s_{3}\right)\right]_{q j}+\left[\boldsymbol{G}_{H}\left(t_{f}-s_{2}\right)\right]_{n q}\left[\boldsymbol{G}_{H}\left(s_{1}-s_{3}\right)\right]_{p j} \\
& +\left[\boldsymbol{G}_{H}\left(t_{f}-s_{3}\right)\right]_{n j}\left[\boldsymbol{G}_{H}\left(s_{1}-s_{2}\right)\right]_{p q}
\end{aligned}
$$

and then (70) becomes

$$
\begin{aligned}
\Gamma_{k l m}^{n r}\left(t_{f}\right) & =\int \ldots \int_{0}^{t_{f}} d s d s_{1} d s_{2} d s_{3}\left[\dot{\boldsymbol{D}}_{2}(s)\right]_{n r}\left\{\left[\boldsymbol{G}_{H}\left(s_{1}\right) \mathfrak{D}\left(s_{1}-s\right)\right]_{n k}\left[\mathfrak{D}\left(s_{2}-s\right) \boldsymbol{G}_{H}\left(s_{3}-s_{2}\right) \mathfrak{D}\left(s_{3}-s\right)\right]_{l m}\right. \\
& +\left[\boldsymbol{G}_{H}\left(s_{2}\right) \mathfrak{D}\left(s_{2}-s\right)\right]_{n l}\left[\mathfrak{D}\left(s_{1}-s\right) \boldsymbol{G}_{H}\left(s_{3}-s_{2}\right) \mathfrak{D}\left(s_{3}-s\right)\right]_{k m} \\
& \left.+\left[\boldsymbol{G}_{H}\left(s_{3}\right) \mathfrak{D}\left(s_{3}-s\right)\right]_{n m}\left[\mathfrak{D}\left(s_{1}-s\right) \boldsymbol{G}_{H}\left(s_{2}-s_{1}\right) \mathfrak{D}\left(s_{2}-s\right)\right]_{k l}\right\}
\end{aligned}
$$


after we make the changes of variables $t_{f}-s \rightarrow s, t_{f}-s^{\prime} \rightarrow s^{\prime}$, and $t_{f}-s_{i} \rightarrow s_{i}$. We use the short hand notation [ $\left.\cdots\right]$ to represent each of the three terms in the integrand in Eq. (72). Because of the property $\mathfrak{D}\left(s_{i}-s\right)=0$ for $s_{i} \leq s$, we may write

$$
\int \cdots \int_{0}^{t_{f}}[\cdots] d s d s_{1} d s_{2} d s_{3}=\int_{0}^{t_{f}} d s \int_{-\infty}^{t_{f}} d s_{1} \int_{0}^{t_{f}} d s_{2} \int_{0}^{t_{f}} d s_{3}[\cdots]=\int_{0}^{t_{f}} d s \int_{-\infty}^{t_{f}} \prod_{i=1}^{3} d s_{i}[\cdots] .
$$

Note that the lower limit of the integration over $s_{i},(i=1,2,3)$ in Eq. (72) is zero while in Eq. (73) is $-\infty$. Finally, one can change the lower limit for the $s$ integral in Eq. (72) from 0 to $-\infty$ by replacing $\left[\dot{\boldsymbol{D}}_{2}(s)\right]_{n r}$ with $[\mathfrak{D}(s)]_{n r}$. Hence it leads to,

$$
\begin{aligned}
\Gamma_{k l m}^{n r}\left(t_{f}\right) & =\int \cdots \int_{-\infty}^{t_{f}} d s d s_{1} d s_{2} d s_{3}[\mathfrak{D}(s)]_{n r}\left\{\left[\boldsymbol{G}_{H}\left(s_{1}\right) \mathfrak{D}\left(s_{1}-s\right)\right]_{n k}\left[\mathfrak{D}\left(s_{2}-s\right) \boldsymbol{G}_{H}\left(s_{3}-s_{2}\right) \mathfrak{D}\left(s_{3}-s\right)\right]_{l m}\right. \\
& +\left[\boldsymbol{G}_{H}\left(s_{2}\right) \mathfrak{D}\left(s_{2}-s\right)\right]_{n l}\left[\mathfrak{D}\left(s_{1}-s\right) \boldsymbol{G}_{H}\left(s_{3}-s_{2}\right) \mathfrak{D}\left(s_{3}-s\right)\right]_{k m} \\
& \left.+\left[\boldsymbol{G}_{H}\left(s_{3}\right) \mathfrak{D}\left(s_{3}-s\right)\right]_{n m}\left[\mathfrak{D}\left(s_{1}-s\right) \boldsymbol{G}_{H}\left(s_{2}-s_{1}\right) \mathfrak{D}\left(s_{2}-s\right)\right]_{k l}\right\}
\end{aligned}
$$

Next we compute $\tilde{\Gamma}_{k l m}^{n r}\left(t_{f}\right)$. Since

$$
\left.\frac{\partial^{2} \delta \mathcal{Z}_{\xi}^{(0)}\left[\boldsymbol{j}, \boldsymbol{j}^{\prime}, \boldsymbol{h}\right)}{\partial h_{n}^{2} \delta j_{r}^{\prime}(s)}\right|_{\dot{j}^{\prime}=\boldsymbol{h}=0}=-2 \sum_{p} \int_{0}^{t_{f}} d s^{\prime}\left[\dot{\boldsymbol{D}}_{2}\left(t_{f}-s\right)\right]_{n r}\left[\dot{\boldsymbol{D}}_{2}\left(t_{f}-s^{\prime}\right)\right]_{n p} \xi_{p}\left(s^{\prime}\right) \mathscr{Z}^{(0)}\left[\varrho_{i}, \boldsymbol{j}\right] \exp \left\{i \overline{\mathscr{S}}_{\xi}^{(0)}[\boldsymbol{j}, \boldsymbol{h}=0)\right\}
$$

where the factor 2 comes from the fact the derivatives $\partial h_{n}$ can be applied to either $\overline{\mathscr{S}}_{\xi}^{(0)}[\boldsymbol{j}, \boldsymbol{h})$ or $\mathscr{Z}^{(0)}\left[\varrho_{i}, \boldsymbol{j}\right]$. Then we find

$$
\tilde{\Gamma}_{k l m}^{n r}\left(t_{f}\right)=2 i \sum_{p} \int_{0}^{t_{f}} d s \int_{0}^{t_{f}} d s^{\prime}\left\langle\left.\left[\dot{\boldsymbol{D}}_{2}\left(t_{f}-s\right)\right]_{n r}\left[\dot{\boldsymbol{D}}_{2}\left(t_{f}-s^{\prime}\right)\right]_{n p} \xi_{p}\left(s^{\prime}\right) \frac{\delta^{3} \mathscr{Z}^{(0)}\left[\varrho_{i}, \boldsymbol{j}\right] \exp \left\{i \overline{\mathscr{S}}_{\xi}^{(0)}[\boldsymbol{j}, \boldsymbol{h}=0)\right\}}{\delta j_{k}(s) \delta j_{l}(s) \delta j_{m}(s)}\right|_{\boldsymbol{j}=0}\right\rangle
$$

Similar to the evaluation of $\Gamma_{k l m}^{n r}\left(t_{f}\right)$, Theorem 9 in Appendix D shows that the only nonzero contribution in the late time limit $t_{f} \rightarrow \infty$ comes from the case when all three functional derivatives act only on $\exp \left\{i \overline{\mathscr{S}}_{\xi}^{(0)}[\boldsymbol{j}, \boldsymbol{h}=0)\right\}$. Therefore, following the similar steps in Eqs. (70-74), we find

$$
\begin{array}{rl}
\tilde{\Gamma}_{k l m}^{n r}\left(t_{f}\right)=2 \int \ldots \int_{-\infty}^{t_{f}} d s d s^{\prime} d s_{1} d s_{2} & d s_{3}[\mathfrak{D}(s)]_{n r} \\
\times & \left\{\left[\dot{\mathfrak{D}}\left(s^{\prime}\right) \boldsymbol{G}_{H}\left(s_{1}-s^{\prime}\right) \mathfrak{D}\left(s_{1}-s\right)\right]_{n k}\left[\mathfrak{D}\left(s_{2}-s\right) \boldsymbol{G}_{H}\left(s_{3}-s_{2}\right) \mathfrak{D}\left(s_{3}-s\right)\right]_{l m}\right. \\
& +\left[\dot{D}\left(s^{\prime}\right) \boldsymbol{G}_{H}\left(s_{2}-s^{\prime}\right) \mathfrak{D}\left(s_{2}-s\right)\right]_{n l}\left[\mathfrak{D}\left(s_{1}-s\right) \boldsymbol{G}_{H}\left(s_{3}-s_{2}\right) \mathfrak{D}\left(s_{3}-s\right)\right]_{k m} \\
& \left.\left.+\dot{\mathfrak{D}}\left(s^{\prime}\right) \boldsymbol{G}_{H}\left(s_{3}-s^{\prime}\right) \mathfrak{D}\left(s_{3}-s\right)\right]_{n m}\left[\mathfrak{D}\left(s_{1}-s\right) \boldsymbol{G}_{H}\left(s_{2}-s_{1}\right) \mathfrak{D}\left(s_{2}-s\right)\right]_{k l}\right\} .
\end{array}
$$

In the late time limit $t_{f} \rightarrow \infty$, we then find that the first-order corrections from the $\beta$ - and KG-type of nonlinearities to the rates of the energy exchange between the $n^{\text {th }}$ oscillator and its bath are given by

$$
\begin{aligned}
& P_{\xi_{n}}^{(1)}(\infty)=\sum_{k l m r} \mu_{k l m r} \Gamma_{k l m}^{n r}(\infty) \\
& P_{\gamma_{n}}^{(1)}(\infty)=-2 \gamma \sum_{k l m r} \mu_{k l m r} \tilde{\Gamma}_{k l m}^{n r}(\infty)
\end{aligned}
$$

Both heat current approach time-independent constants according to Eq. $(74,77)$ in the late time limit, which signals the existence of the NESS. The diagrammatic representation of $\Gamma_{k l m}^{n r}(\infty)$ and $\tilde{\Gamma}_{k l m}^{n r}(\infty)$ is shown in Fig. 3. 

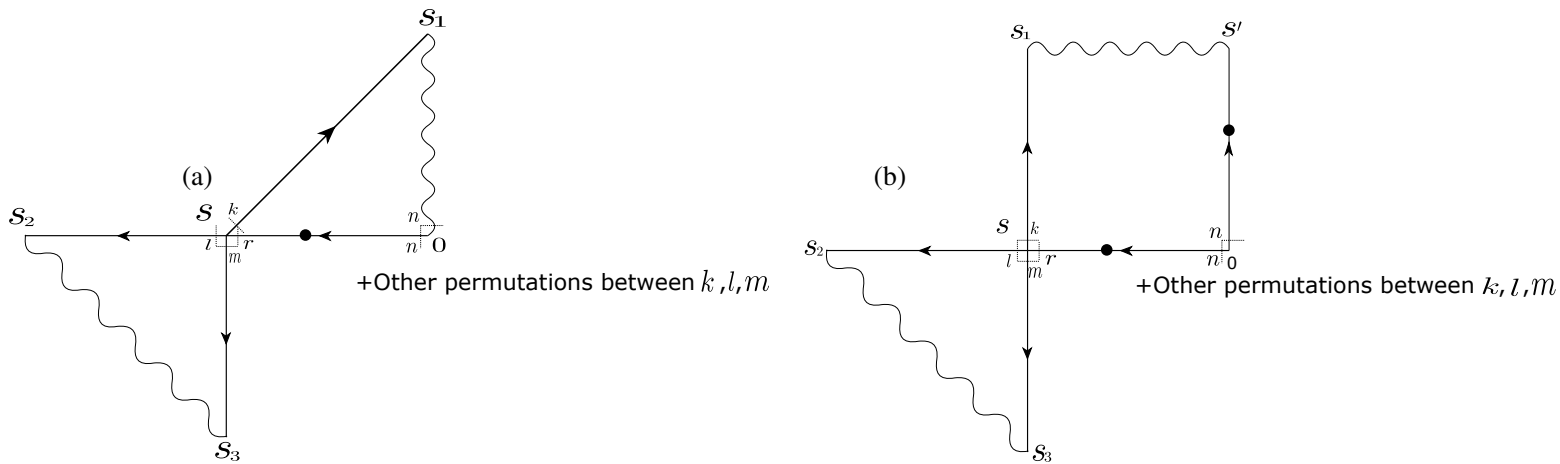

Figure 3: Time-domain diagrammatic representations of $\Gamma_{k l m}^{n r}(\infty)$ and $\tilde{\Gamma}_{k l m}^{n r}(\infty)$ are shown in (a) and (b) respectively. Note that the factor of 2 in Eq. (77) is not shown in (b).

\subsection{2. $\alpha$-FPUT nonlinearity}

Here we consider the corrections caused by the $\alpha$-type of nonlinearity to $P_{\xi_{n}}$ and $P_{\gamma_{n}}$. By Theorem 3 in Appendix $\mathrm{D}$, we recognize

$$
\left.\partial_{n}\left\langle r_{k}(s) q_{l}(s) q_{m}(s)\right\rangle\right|_{h=0}=0 .
$$

Furthermore, following similar steps in Sec. 4.2.1, we find that for $\alpha$-FPUT nonlinearity,

$$
\begin{aligned}
\Gamma_{k l}^{n m}\left(t_{f}\right) & \left.\equiv \lim _{t_{f} \rightarrow \infty} \int_{0}^{t_{f}} d s\left\langle\xi_{n}\left(t_{f}\right) \partial_{n}\left\langle r_{k}(s) r_{l}(s) q_{m}(s)\right\rangle_{0}\right\rangle\right\rangle\left.\right|_{\boldsymbol{h}=0} \\
& =\left.\int_{0}^{t_{f}} d s\left\langle\left[\dot{\boldsymbol{D}}_{2}\left(t_{f}-s\right)\right]_{n m} \xi_{n}\left(t_{f}\right) \frac{\delta^{2} \mathscr{Z}^{(0)}\left[\varrho_{i}, \boldsymbol{j}\right] \exp \left\{i \overline{\mathscr{S}}_{\xi}^{(0)}[\boldsymbol{j}, \boldsymbol{h}=0)\right\}}{\delta j_{k}(s) \delta j_{l}(s)}\right\rangle\right|_{\boldsymbol{j}=0}, \\
\tilde{\Gamma}_{k}^{n l m}\left(t_{f}\right) & \equiv-i \lim _{t_{f} \rightarrow \infty} \int_{0}^{t_{f}} d s\left\langle\left\langle\left.\partial_{n}^{2}\left\langle r_{k}(s) q_{l}(s) q_{m}(s)\right\rangle_{0}\right|_{\boldsymbol{h}=0}\right\rangle\right\rangle \\
& =-2 \int_{0}^{t_{f}} \int_{0}^{t_{f}} d s^{\prime} d s\left\langle\left.\left\langle\left[\dot{\boldsymbol{D}}_{2}\left(t_{f}-s\right)\right]_{n l} \dot{\boldsymbol{D}}_{2}\left(t_{f}-s^{\prime}\right)\right]_{n m} \frac{\delta\left(\mathscr{Z}^{(0)}\left[\varrho_{i}, \boldsymbol{j}\right] \exp \left\{i \overline{\mathscr{S}}_{\boldsymbol{\xi}}^{(0)}[\boldsymbol{j}, \boldsymbol{h}=0)\right\}\right)}{\delta j_{k}(s)}\right|_{\boldsymbol{j}=0}\right\rangle, \\
\bar{\Gamma}_{k l}^{n m}\left(t_{f}\right) & \equiv-i \lim _{t_{f} \rightarrow \infty} \int_{0}^{t_{f}} d s\left\langle\left\langle\left.\partial_{n}^{2}\left\langle r_{k}(s) r_{l}(s) q_{m}(s)\right\rangle_{0}\right|_{\boldsymbol{h}=0}\right\rangle\right\rangle \\
& =2 \sum_{p} \int_{0}^{t_{f}} \int_{0}^{t_{f}} d s^{\prime} d s\left\langle\left.\left\langle\left[\dot{\boldsymbol{D}}_{2}\left(t_{f}-s\right)\right]_{n m} \dot{\boldsymbol{D}}_{2}\left(t_{f}-s^{\prime}\right)\right]_{n p} \xi_{p}\left(s^{\prime}\right) \frac{\delta\left(\mathcal{Z}^{(0)}\left[\varrho_{i}, \boldsymbol{j}\right] \exp \left\{i \overline{\mathscr{S}}_{\xi}^{(0)}[\boldsymbol{j}, \boldsymbol{h}=0)\right\}\right)}{\delta j_{k}(s) \delta j_{l}(s)}\right|_{\boldsymbol{j}=0}\right\rangle .
\end{aligned}
$$

By Theorems 9-11 in Appendix D, we observe that, the contributions from the cases where at least one functional derivatives with respect to $\boldsymbol{j}$ is applied to $\mathscr{Z}^{(0)}\left[\varrho_{i}, \boldsymbol{j}\right]$ vanish in the late time limit $t_{f} \rightarrow \infty$. The only possible nonzero contributions in Eqs. (81-83) can only come from the situation that all functional derivatives with respect to $j$ are applied to $\exp \left\{i \overline{\mathscr{S}}_{\xi}^{(0)}[\boldsymbol{j}, \boldsymbol{h}=0)\right\}$. However, this will generate odd powers in $\boldsymbol{\xi}$. Since $\boldsymbol{\xi}$ is Gaussian, their ensemble averages give zero. Therefore, we arrive

$$
\Gamma_{k l}^{n m}(\infty)=\tilde{\Gamma}_{k}^{n l m}(\infty)=\bar{\Gamma}_{k l}^{n m}(\infty)=0 .
$$

In other words, the powers $P_{\xi_{n}}(\infty)$ and $P_{\gamma_{n}}(\infty)$ do not have first-order corrections in $\alpha$-type nonlinearity.

\subsection{First order correction to the energy current $P_{v \rightarrow n}^{(2)}\left(t_{f}\right)$}

Here we will discuss the nonlinear correction to the inter-oscillator energy flow, contributed by the quadratic coupling $(\eta=2)$ between oscillator $v$ and $n$, i.e., between oscillator 2 and oscillator 1 . The correction actually stems from the nonlinearity in the generating function. We first discuss the $\beta$ - and KG-type nonlinearities. From (33), we see that we need to compute the the first-order correction, $w_{v \rightarrow n}^{(1,2)}$, of $w_{v \rightarrow n}^{(2)}$. 


\subsection{1. $K G$ and $\beta$-FPUT nonlinearities}

According to Eqs. $(36,41)$, the first-order corrections of $w_{v \rightarrow n}^{(2)}\left(t_{f}, \boldsymbol{\xi}\right]$ due to the $\beta$ - and KG-type nonlinearities are given by

$$
\begin{aligned}
w_{v \rightarrow n}^{(1,2)}\left(t_{f}, \boldsymbol{\xi}\right] & =-\sum_{k l m r} \int_{0}^{t_{f}} d s\left[\left.\sigma_{k l m r}\left(\partial_{n}\left\langle r_{n}\left(t_{f}\right) r_{k}(s) q_{l}(s) q_{m}(s) q_{r}(s)\right\rangle_{0}-\partial_{n}\left\langle r_{v}\left(t_{f}\right) r_{k}(s) q_{l}(s) q_{m}(s) q_{r}(s)\right\rangle_{0}\right)\right|_{h=0}\right. \\
& \left.+\left.\mu_{k l m r}\left(\partial_{n}\left\langle r_{n}\left(t_{f}\right) r_{k}(s) q_{l}(s) q_{m}(s) q_{r}(s)\right\rangle_{0}-\partial_{n}\left\langle r_{v}\left(t_{f}\right) r_{k}(s) r_{l}(s) r_{m}(s) q_{r}(s)\right\rangle_{0}\right)\right|_{\boldsymbol{h}=0}\right],
\end{aligned}
$$

where

$$
\begin{aligned}
\left.\partial_{n}\left\langle r_{v}\left(t_{f}\right) r_{k}(s) q_{l}(s) q_{m}(s) q_{r}(s)\right\rangle_{0}\right|_{\boldsymbol{h}=0} & =-\left.i \frac{\partial \delta^{5} \mathcal{Z}_{\xi}^{(0)}\left[\boldsymbol{j}, \boldsymbol{j}^{\prime}, \boldsymbol{h}\right)}{\partial h_{n} \delta j_{v}\left(t_{f}\right) \delta j_{k}(s) \delta j_{l}^{\prime}(s) \delta j_{m}^{\prime}(s) \delta j_{r}^{\prime}(s)}\right|_{\boldsymbol{j}=\boldsymbol{j}^{\prime}=\boldsymbol{h}=0}, \\
\left.\partial_{n}\left\langle r_{v}\left(t_{f}\right) r_{k}(s) r_{l}(s) r_{m}(s) q_{r}(s)\right\rangle_{0}\right|_{\boldsymbol{h}=0} & =-\left.i \frac{\partial \delta^{5} \mathcal{Z}_{\xi}^{(0)}\left[\boldsymbol{j}, \boldsymbol{j}^{\prime}, \boldsymbol{h}\right)}{\partial h_{n} \delta j_{v}\left(t_{f}\right) \delta j_{k}(s) \delta j_{l}(s) \delta j_{m}(s) \delta j_{r}^{\prime}(s)}\right|_{\boldsymbol{j}=\boldsymbol{j}^{\prime}=\boldsymbol{h}=0} .
\end{aligned}
$$

Theorem 4 indicates that Eq. (86) vanishes, so we need to evaluate only Eq. (87). So one only needs to evaluate Eq. (87). After performing the derivatives $\partial \delta^{2} / \partial h_{n} \delta j_{v}\left(t_{f}\right) \delta j_{r}^{\prime}(s)$, we find

$$
\begin{gathered}
\left.\partial_{n}\left\langle r_{v}\left(t_{f}\right) r_{k}(s) r_{l}(s) r_{m}(s) q_{r}(s)\right\rangle_{0}\right|_{\boldsymbol{h}=0}=-i \times \\
\frac{\delta^{3}}{\delta j_{k}(s) \delta j_{l}(s) \delta j_{m}(s)}\left(\frac{\delta\left(\mathscr{Z}^{(0)}\left[\varrho_{i}, \boldsymbol{j}\right] \exp \left\{i \overline{\mathscr{S}}_{\xi}^{(0)}[\boldsymbol{j}, \boldsymbol{h})\right\}\right)}{\delta j_{v}\left(t_{f}\right)} \frac{\partial \delta\left(\exp \left\{i \bar{\Xi}_{0 \xi}\left[\boldsymbol{j}, \boldsymbol{j}^{\prime} \boldsymbol{h}\right)\right\}\right)}{\partial h_{n} \delta j_{r}^{\prime}(s)}\right. \\
\left.+\frac{\partial\left(\mathscr{Z}^{(0)}\left[\varrho_{i}, \boldsymbol{j}\right] \exp \left\{i \overline{\mathscr{S}}_{\xi}^{(0)}[\boldsymbol{j}, \boldsymbol{h})\right\}\right)}{\partial h_{n}} \frac{\delta^{2}\left(\exp \left\{i \bar{\Xi}_{0 \xi}\left[\boldsymbol{j}, \boldsymbol{j}^{\prime} \boldsymbol{h}\right)\right\}\right)}{\delta j_{v}\left(t_{f}\right) \delta j_{r}^{\prime}(s)}+\cdots\right)\left.\right|_{\boldsymbol{j}=\boldsymbol{j}^{\prime}=\boldsymbol{h}=0}
\end{gathered}
$$

where all other omitted terms vanish in the end due to according Theorems 1 and 3 . As one can see from Theorem 5 in Appendix D, if the functional derivative $\delta / \delta j_{v}\left(t_{f}\right)$ in the first term of the right hand side acts on $\mathscr{Z}^{(0)}\left[\varrho_{i}, j\right]$, it will generates terms that will vanish in the late time limit $t_{f} \rightarrow \infty$. Therefore, the only possible non-vanishing terms come from when $\delta / \delta j_{v}\left(t_{f}\right)$ acts on $\exp \left\{i \overline{\mathscr{S}}_{\xi}^{(0)}[\boldsymbol{j}, \boldsymbol{h}=0)\right\}$. With this observation, we find

$$
\begin{gathered}
\left.\partial_{n}\left\langle r_{v}\left(t_{f}\right) r_{k}(s) r_{l}(s) r_{m}(s) q_{r}(s)\right\rangle_{0}\right|_{\boldsymbol{h}=0}=i \times \\
\left\{\sum_{p} \int_{0}^{t_{f}} d s^{\prime}\left[\dot{\boldsymbol{D}}_{2}\left(t_{f}-s\right)\right]_{n r} \mathfrak{D}\left(t_{f}-s^{\prime}\right)\right]_{v p} \xi_{p}\left(s^{\prime}\right) \frac{\delta^{3}\left(\mathscr{Z}^{(0)}\left[\varrho_{i}, \boldsymbol{j}\right] \exp \left\{i \overline{\mathscr{S}}_{\xi}^{(0)}[\boldsymbol{j}, \boldsymbol{h}=0)\right\}\right)}{\delta j_{k}(s) \delta j_{l}(s) \delta j_{m}(s)} \\
\left.+\sum_{p} \int_{0}^{t_{f}} d s^{\prime}\left[\mathfrak{D}\left(t_{f}-s\right)\right]_{v r}\left[\dot{\boldsymbol{D}}_{2}\left(t_{f}-s^{\prime}\right)\right]_{n p} \xi_{p}\left(s^{\prime}\right) \frac{\delta^{3}\left(\mathscr{Z}^{(0)}\left[\varrho_{i}, \boldsymbol{j}\right] \exp \left\{i \overline{\mathscr{S}}_{\xi}^{(0)}[\boldsymbol{j}, \boldsymbol{h}=0)\right\}\right)}{\delta j_{k}(s) \delta j_{l}(s) \delta j_{m}(s)}\right\} .
\end{gathered}
$$

Let us define

$$
\begin{aligned}
& \Upsilon_{v k l m}^{n r}\left(t_{f}\right) \equiv \lim _{t_{f} \rightarrow \infty} i \sum_{p} \int_{0}^{t_{f}} \int_{0}^{t_{f}} d s d s^{\prime}\left\langle\left\langle\left[\dot{\boldsymbol{D}}_{2}\left(t_{f}-s\right)\right]_{n r} \mathfrak{D}\left(t_{f}-s^{\prime}\right)\right]_{v p} \xi_{p}\left(s^{\prime}\right) \frac{\delta^{3}\left(\mathscr{Z}^{(0)}\left[\varrho_{i}, \boldsymbol{j}\right] \exp \left\{i \overline{\mathscr{S}}_{\xi}^{(0)}[\boldsymbol{j}, \boldsymbol{h}=0)\right\}\right)}{\delta j_{k}(s) \delta j_{l}(s) \delta j_{m}(s)}\right\rangle, \\
& \tilde{\Upsilon}_{v k l m}^{n r}\left(t_{f}\right) \equiv \lim _{t_{f} \rightarrow \infty} i \sum_{p} \int_{0}^{t_{f}} \int_{0}^{t_{f}} d s d s^{\prime}\left\langle\left\langle\left[\mathfrak{D}\left(t_{f}-s\right)\right]_{v r}\left[\dot{\boldsymbol{D}}_{2}\left(t_{f}-s^{\prime}\right)\right]_{n p} \xi_{p}\left(s^{\prime}\right) \frac{\delta^{3}\left(\mathscr{Z}^{(0)}\left[\varrho_{i}, \boldsymbol{j}\right] \exp \left\{i \overline{\mathscr{S}}_{\xi}^{(0)}[\boldsymbol{j}, \boldsymbol{h}=0)\right\}\right)}{\delta j_{k}(s) \delta j_{l}(s) \delta j_{m}(s)}\right\rangle,\right.
\end{aligned}
$$

then

$$
\lim _{t_{f} \rightarrow \infty} \int_{0}^{t_{f}} d s\left\langle\left\langle\left.\partial_{n}\left\langle r_{v}\left(t_{f}\right) r_{k}(s) r_{l}(s) r_{m}(s) q_{r}(s)\right\rangle_{0}\right|_{h=0}\right\rangle\right\rangle=\Upsilon_{v k l m}^{n r}\left(t_{f}\right)+\tilde{\Upsilon}_{v k l m}^{n r}\left(t_{f}\right)
$$


We have justified in Theorems 9 in Appendix $\mathrm{D}$ that in the late time limit $t_{f} \rightarrow \infty$, the only non-vanishing terms come from the contribution when all the three functional derivatives with respect to $\boldsymbol{j}$ acts on $\exp \left\{i \overline{\mathscr{S}}_{\xi}^{(0)}[\boldsymbol{j}, \boldsymbol{h}=0)\right\}$. Therefore, similar with the calculations in Eqs. (70-72), we obtain

$$
\begin{aligned}
\Upsilon_{v k l m}^{n r}\left(t_{f}\right) & \left.=\int \cdots \int_{0}^{t_{f}} d s d s^{\prime} d s_{1} d s_{2} d s_{3} \dot{\boldsymbol{D}}_{2}\left(t_{f}-s\right)\right]_{n r} \\
& \times\left\{\left[\mathfrak{D}\left(t_{f}-s^{\prime}\right) \boldsymbol{G}_{H}\left(s^{\prime}-s_{1}\right) \mathfrak{D}\left(s-s_{1}\right)\right]_{v k}\left[\mathfrak{D}\left(s-s_{2}\right) \boldsymbol{G}_{H}\left(s_{2}-s_{3}\right) \mathfrak{D}\left(s-s_{3}\right)\right]_{l m}\right. \\
& +\left[\mathfrak{D}\left(t_{f}-s\right) \boldsymbol{G}_{H}\left(s^{\prime}-s_{2}\right) \mathfrak{D}\left(s-s_{2}\right)\right]_{v l}\left[\mathfrak{D}\left(s-s_{1}\right) \boldsymbol{G}_{H}\left(s_{1}-s_{3}\right) \mathfrak{D}\left(s-s_{3}\right)\right]_{k m} \\
& \left.+\left[\mathfrak{D}\left(t_{f}-s\right) \boldsymbol{G}_{H}\left(s^{\prime}-s_{3}\right) \mathfrak{D}\left(s-s_{3}\right)\right]_{v m}\left[\mathfrak{D}\left(s-s_{1}\right) \boldsymbol{G}_{H}\left(s_{1}-s_{2}\right) \mathfrak{D}\left(s-s_{2}\right)\right]_{k l}\right\}, \\
\tilde{\Upsilon}_{v k l m}^{n r}\left(t_{f}\right) & =\int \cdots \int_{0}^{t_{f}} d s d s^{\prime} d s_{1} d s_{2} d s_{3}\left[\mathfrak{D}\left(t_{f}-s\right)\right]_{v r} \\
& \times\left\{\left[\dot{\boldsymbol{D}}_{2}\left(t_{f}-s^{\prime}\right) \boldsymbol{G}_{H}\left(s^{\prime}-s_{1}\right) \mathfrak{D}\left(s-s_{1}\right)\right]_{n k}\left[\mathfrak{D}\left(s-s_{2}\right) \boldsymbol{G}_{H}\left(s_{2}-s_{3}\right) \mathfrak{D}\left(s-s_{3}\right)\right]_{l m}\right. \\
& \left.+\left[\dot{\boldsymbol{D}}_{2}\left(t_{f}-s\right)\right] \boldsymbol{G}_{H}\left(s^{\prime}-s_{2}\right) \mathfrak{D}\left(s-s_{2}\right)\right]_{n l}\left[\mathfrak{D}\left(s-s_{1}\right) \boldsymbol{G}_{H}\left(s_{1}-s_{3}\right) \mathfrak{D}\left(s-s_{3}\right)\right]_{k m} \\
& \left.+\left[\dot{\boldsymbol{D}}_{2}\left(t_{f}-s\right) \boldsymbol{G}_{H}\left(s^{\prime}-s_{3}\right) \mathfrak{D}\left(s-s_{3}\right)\right]_{n m}\left[\mathfrak{D}\left(s-s_{1}\right) \boldsymbol{G}_{H}\left(s_{1}-s_{2}\right) \mathfrak{D}\left(s-s_{2}\right)\right]_{k l}\right\} .
\end{aligned}
$$

Making change of variables $t_{f}-s \rightarrow s, t_{f}-s^{\prime} \rightarrow s^{\prime}$, and $t_{f}-s_{i} \rightarrow s_{i}$ and employing the trick given in (73), we arrive at

$$
\begin{aligned}
\Upsilon_{v k l m}^{n r}\left(t_{f}\right) & =\int \cdots \int_{-\infty}^{t_{f}} d s d s^{\prime} d s_{1} d s_{2} d s_{3}[\mathfrak{D}(s)]_{n r} \\
& \times\left\{\left[\mathfrak{D}\left(s^{\prime}\right) \boldsymbol{G}_{H}\left(s_{1}-s^{\prime}\right) \mathfrak{D}\left(s_{1}-s\right)\right]_{v k}\left[\mathfrak{D}\left(s_{2}-s\right) \boldsymbol{G}_{H}\left(s_{3}-s_{2}\right) \mathfrak{D}\left(s_{3}-s\right)\right]_{l m}\right. \\
& +\left[\mathfrak{D}(s) \boldsymbol{G}_{H}\left(s_{2}-s^{\prime}\right) \mathfrak{D}\left(s_{2}-s\right)\right]_{v l}\left[\mathfrak{D}\left(s_{1}-s\right) \boldsymbol{G}_{H}\left(s_{3}-s_{1}\right) \mathfrak{D}\left(s_{3}-s\right)\right]_{k m} \\
& \left.+\left[\mathfrak{D}(s) \boldsymbol{G}_{H}\left(s_{3}-s^{\prime}\right) \mathfrak{D}\left(s_{3}-s\right)\right]_{v m}\left[\mathfrak{D}\left(s_{1}-s\right) \boldsymbol{G}_{H}\left(s_{2}-s_{1}\right) \mathfrak{D}\left(s_{2}-s\right)\right]_{k l}\right\}, \\
\tilde{\Upsilon}_{v k l m}^{n r}\left(t_{f}\right) & =\int \cdots \int_{-\infty}^{t_{f}} d s d s^{\prime} d s_{1} d s_{2} d s_{3}[\mathfrak{D}(s)]_{v r} \\
& \times\left\{\left[\dot{D}\left(s^{\prime}\right) \boldsymbol{G}_{H}\left(s_{1}-s^{\prime}\right) \mathfrak{D}\left(s_{1}-s\right)\right]_{n k}\left[\mathfrak{D}\left(s_{2}-s\right) \boldsymbol{G}_{H}\left(s_{3}-s_{2}\right) \mathfrak{D}\left(s_{3}-s\right)\right]_{l m}\right. \\
& +\left[\mathfrak{D}(s) \boldsymbol{G}_{H}\left(s_{2}-s^{\prime}\right) \mathfrak{D}\left(s_{2}-s\right)\right]_{n l}\left[\mathfrak{D}\left(s_{1}-s\right) \boldsymbol{G}_{H}\left(s_{3}-s_{1}\right) \mathfrak{D}\left(s_{3}-s\right)\right]_{k m} \\
& \left.+\left[\mathfrak{D}(s) \boldsymbol{G}_{H}\left(s_{2}-s^{\prime}\right) \mathfrak{D}\left(s_{3}-s\right)\right]_{n m}\left[\mathfrak{D}\left(s_{1}-s\right) \boldsymbol{G}_{H}\left(s_{2}-s_{1}\right) \mathfrak{D}\left(s_{2}-s\right)\right]_{k l}\right\} .
\end{aligned}
$$

Now we take the limit $t_{f} \rightarrow \infty$, we find

$$
w_{v \rightarrow n}^{(1,2)}(\infty)=-\sum_{k l m r} \mu_{k l m r}\left[\Upsilon_{n k l m}^{n r}(\infty)+\tilde{\Upsilon}_{n k l m}^{n r}(\infty)-\Upsilon_{v k l m}^{n r}(\infty)-\tilde{\Upsilon}_{v k l m}^{n r}(\infty)\right]
$$

This is one of the the expressions we need to find the corrections to the energy flow contributed by the quadratic coupling $(\eta=2)$ between oscillator $v$ and $n$. Next, we discuss the corresponding contribution from the $\alpha$-type nonlinearity in the generating functional.

\subsection{2. $\alpha$-FPUT nonlinearity}

To compute the first-order correction to $w_{v \rightarrow n}^{(2)}\left(t_{f}, \xi\right]$ due to $\alpha$-FPUT nonlinearity in the generating functional, we need to evaluate the following quantities

$$
\begin{aligned}
& \left.\partial_{n}\left\langle r_{v}\left(t_{f}\right) r_{k}(s) q_{l}(s) q_{m}(s)\right\rangle_{0}\right|_{\boldsymbol{h}=0}=\left.\frac{\partial \delta^{4} \mathcal{Z}_{\xi}^{(0)}\left[\boldsymbol{j}, \boldsymbol{j}^{\prime}, \boldsymbol{h}\right)}{\partial h_{n} \delta j_{v}\left(t_{f}\right) \delta j_{k}(s) \delta j_{l}^{\prime}(s) \delta j_{m}^{\prime}(s)}\right|_{j=j^{\prime}=\boldsymbol{h}=0}, \\
& \left.\partial_{n}\left\langle r_{v}\left(t_{f}\right) r_{k}(s) r_{l}(s) q_{m}(s)\right\rangle_{0}\right|_{\boldsymbol{h}=0}=\left.\frac{\partial \delta^{4} \mathcal{Z}_{\xi}^{(0)}\left[\boldsymbol{j}, \boldsymbol{j}^{\prime}, \boldsymbol{h}\right)}{\partial h_{n} \delta j_{v}\left(t_{f}\right) \delta j_{k}(s) \delta j_{l}(s) \delta j_{m}^{\prime}(s)}\right|_{\boldsymbol{j}=\boldsymbol{j}^{\prime}=\boldsymbol{h}=0} .
\end{aligned}
$$



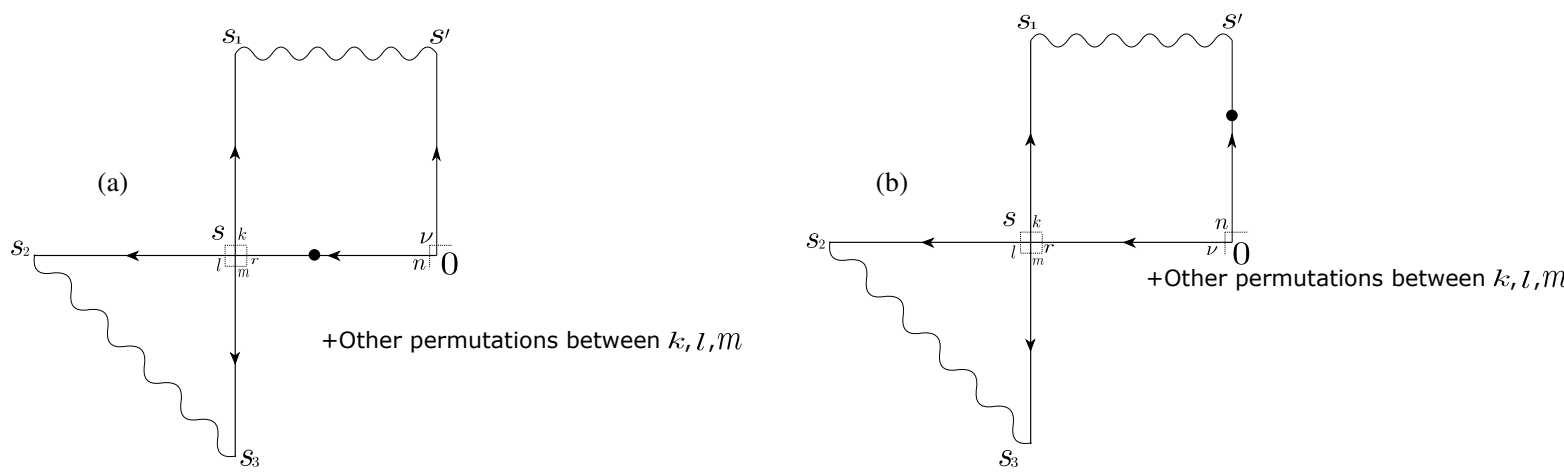

Figure 4: Time-domain diagrammatic representation of $\Upsilon_{k l m}^{n r}(\infty)$ and $\tilde{\Upsilon}_{v k l m}^{n r}(\infty)$

Carrying out the derivatives gives

$$
\begin{aligned}
& \left.\partial_{n}\left\langle r_{v}\left(t_{f}\right) r_{k}(s) q_{l}(s) q_{m}(s)\right\rangle_{0}\right|_{\boldsymbol{h}=0} \\
= & \left.\frac{\delta}{\delta j_{k}(s)}\left(\mathscr{Z}_{0}\left[\varrho_{i}, \boldsymbol{j}\right] \exp \left\{i \overline{\mathscr{S}}_{\xi}^{(0)}[\boldsymbol{j}, \boldsymbol{h})\right\} \frac{\partial \delta^{3}\left(\exp \left\{i \bar{\Xi}_{0 \xi}\left[\boldsymbol{j}, \boldsymbol{j}^{\prime} \boldsymbol{h}\right)\right\}\right)}{\partial h_{n} \delta j_{v}\left(t_{f}\right) \delta j_{l}\left(s^{\prime}\right) \delta j_{m}^{\prime}(s)}+\cdots\right)\right|_{\boldsymbol{j}=j^{\prime}=\boldsymbol{h}=0}, \\
& \left.\partial_{n}\left\langle r_{v}\left(t_{f}\right) r_{k}(s) r_{l}(s) q_{m}(s)\right\rangle_{0}\right|_{\boldsymbol{h}=0} \\
= & \frac{\delta^{2}}{\delta j_{k}(s) \delta j_{l}(s)}\left(\frac{\delta\left(\mathscr{Z}^{(0)}\left[\varrho_{i}, \boldsymbol{j}\right] \exp \left\{i \overline{\mathscr{S}}_{\xi}^{(0)}[\boldsymbol{j}, \boldsymbol{h})\right\}\right)}{\delta j_{v}\left(t_{f}\right)} \frac{\partial \delta\left(\exp \left\{i \bar{\Xi}_{0 \xi}\left[\boldsymbol{j}, \boldsymbol{j}^{\prime} \boldsymbol{h}\right)\right\}\right)}{\partial h_{n} \delta j_{m}^{\prime}(s)}\right. \\
+ & \left.\frac{\partial\left(\mathscr{Z}^{(0)}\left[\varrho_{i}, \boldsymbol{j}\right] \exp \left\{i \overline{\mathscr{S}}_{\xi}^{(0)}[\boldsymbol{j}, \boldsymbol{h})\right\}\right)}{\partial h_{n}} \frac{\delta^{2}\left(\exp \left\{i \bar{\Xi}_{0 \xi}\left[\boldsymbol{j}, \boldsymbol{j}^{\prime} \boldsymbol{h}\right)\right\}\right)}{\delta j_{v}\left(t_{f}\right) \delta j_{m}^{\prime}(s)}+\cdots\right)\left.\right|_{\boldsymbol{j}=\boldsymbol{j}^{\prime}=\boldsymbol{h}=0},
\end{aligned}
$$

where all other omitted terms will vanish in the end due to Theorems 1 and 3. Similar to derivations of Eqs. (81-83), further simplifications give

$$
\begin{aligned}
\int_{0}^{t_{f}} d s\left\langle\left\langle\partial _ { n } \left\langle r_{v}\left(t_{f}\right)\right.\right.\right. & \left.\left.r_{k}(s) q_{l}(s) q_{m}(s)\right\rangle_{0}\right\rangle\left.\right|_{\boldsymbol{h}=0} \\
& \sim \int_{0}^{t_{f}} d s \int_{0}^{t_{f}} d s^{\prime}\left\langle\left\langle\dot{D}_{2}\left(t_{f}-s^{\prime}\right) \mathcal{D}_{2}\left(t_{f}-s\right) \frac{\delta \mathscr{Z}^{(0)}\left[\varrho_{i}, \boldsymbol{j}\right] \exp \left\{i \overline{\mathscr{S}}_{\xi}^{(0)}[\boldsymbol{j}, \boldsymbol{h})\right\}}{\delta j_{k}(s)}\right\rangle,\right. \\
\left.\int_{0}^{t_{f}} d s\left\langle\left\langle\partial_{n}\left\langle r_{v}\left(t_{f}\right) r_{k}(s) r_{l}(s) q_{m}(s)\right\rangle_{0}\right\rangle\right\rangle\right|_{\boldsymbol{h}=0} & \\
& \sim \int_{0}^{t_{f}} d s \int_{0}^{t_{f}} d s^{\prime}\left\langle\dot{D}_{2}\left(t_{f}-s\right) \mathfrak{D}\left(t_{f}-s^{\prime}\right) \xi\left(s^{\prime}\right) \frac{\delta^{2} \mathscr{Z}^{(0)}\left[\varrho_{i}, \boldsymbol{j}\right] \exp \left\{i \overline{\mathscr{S}}_{\xi}^{(0)}[\boldsymbol{j}, \boldsymbol{h})\right\}}{\delta j_{k}(s) j_{l}(s)}\right\rangle \\
& +\int_{0}^{t_{f}} d s \int_{0}^{t_{f}} d s^{\prime}\left\langle\left\langle\mathcal{D}\left(t_{f}-s\right) \dot{D}_{2}\left(t_{f}-s^{\prime}\right) \xi\left(s^{\prime}\right) \frac{\delta^{2} \mathscr{Z}^{(0)}\left[\varrho_{i}, \boldsymbol{j}\right] \exp \left\{i \overline{\mathscr{S}}_{\xi}^{(0)}[\boldsymbol{j}, \boldsymbol{h})\right\}}{\delta j_{k}(s) j_{l}(s)}\right\rangle\right.
\end{aligned}
$$

where we have used $\sim$ notation to indicate that the matrix indices are suppressed on the right hand side, and $\dot{D}_{2}$ and $\mathfrak{D}$ stand for the matrix elements of $\boldsymbol{D}_{2}$ and $\mathfrak{D}$ respectively. According to Theorems 10 and 11, we immediately see that Eqs. $(102,103)$ will vanish at late times $t_{f} \rightarrow \infty$, so we find that

$$
w_{v \rightarrow n}^{(1,2)}(\infty, \boldsymbol{\xi}]=0 .
$$

The $\alpha$-FPUT nonlinearity in the generating functional does not contribute to $P_{v \rightarrow n}^{(1,2)}(\infty)$ between the two oscillators, which results solely from the quadratic intra-oscillator coupling (97). 
4.4. The first order correction to the energy flux $P_{v \rightarrow n}^{(\eta)}\left(t_{f}\right)$ for $\eta \geq 3$

Now we will focus on the contributions to the energy flows between oscillators purely from the nonlinear intraoscillator couplings. Since we only consider nonlinear effects up to first order of $P_{v \rightarrow n}^{(\eta)}\left(t_{f}\right)$, from Eq. (33), we need to compute $w_{v \rightarrow n}^{(0, \eta)}\left(t_{f}\right)$, the zeroth order of $w_{v \rightarrow n}^{(\eta)}\left(t_{f}\right)$, which only involves the linear term in the generating function. The superscript 0 reminds the fact that we use the zeroth-order generating functional $\mathcal{Z}_{\xi}^{(0)}\left[\boldsymbol{j}, \boldsymbol{j}^{\prime}, \boldsymbol{h}\right)$. The quantity $w_{v \rightarrow n}^{(0, n)}\left(t_{f}\right)$ is given by

$$
w_{v \rightarrow n}^{(0, \eta)}\left(t_{f}\right)\left(t_{f}, \boldsymbol{\xi}\right]=-\left.(-i)^{\eta} \frac{\partial}{\partial h_{n}}\left(\frac{\delta}{\delta j_{n}\left(t_{f}\right)}-\frac{\delta}{\delta j_{v}\left(t_{f}\right)}\right)^{\eta-1} \mathcal{Z}_{\xi}^{(0)}\left[\boldsymbol{j}, \boldsymbol{j}^{\prime}, \boldsymbol{h}\right)\right|_{j=j^{\prime}=\boldsymbol{h}=0}
$$

where

$$
\left.\mathcal{Z}_{\xi}^{(0)}\left[\boldsymbol{j}, \boldsymbol{j}^{\prime}, \boldsymbol{h}\right)\right|_{\boldsymbol{j}^{\prime}=0}=\mathscr{Z}^{(0)}\left[\rho_{i}, \boldsymbol{j}\right] \exp \left\{i \overline{\mathscr{S}}_{\xi}^{(0)}[\boldsymbol{j}, \boldsymbol{h})\right\}
$$

From Theorem 5, we know in the late time limit $t_{f} \rightarrow \infty$, the contributions to $w_{v \rightarrow n}^{(0, \eta)}\left(t_{f}, \boldsymbol{\xi}\right.$ ] come from the case when all the functional derivatives with respect to $\boldsymbol{j}\left(t_{f}\right)$ act on $\exp \left\{i \overline{\mathscr{S}}_{\xi}^{(0)}[\boldsymbol{j}, \boldsymbol{h})\right\}$. Therefore, we find

$$
w_{v \rightarrow n}^{(0, \eta)}\left(t_{f}, \boldsymbol{\xi}\right]=-\left.(-i)^{n} \frac{\partial}{\partial h_{n}}\left(\frac{\delta}{\delta j_{n}\left(t_{f}\right)}-\frac{\delta}{\delta j_{v}\left(t_{f}\right)}\right)^{\eta-1} \exp \left\{i \overline{\mathscr{S}}_{\xi}^{(0)}[\boldsymbol{j}, \boldsymbol{h})\right\}\right|_{\boldsymbol{j}=\boldsymbol{h}=0} .
$$

For $\eta=3$, after we Taylor-expand the righthand side, we obtain

$$
\begin{aligned}
& w_{v \rightarrow n,}^{(0,3)}\left(t_{f}, \boldsymbol{\xi}\right] \\
= & -\left.\frac{1}{2 !} \frac{\partial}{\partial h_{n}}\left(\frac{\delta}{\delta j_{n}\left(t_{f}\right)}-\frac{\delta}{\delta j_{v}\left(t_{f}\right)}\right)^{2}\left[\boldsymbol{h}^{T} \cdot \int_{0}^{t_{f}} d s \dot{\boldsymbol{D}}_{2}\left(t_{f}-s\right) \cdot \boldsymbol{\xi}(s)\right]\left[\int_{0}^{t_{f}} d s \int_{0}^{t_{f}} d s^{\prime} \boldsymbol{j}^{T}(s) \cdot \mathfrak{D}\left(s-s^{\prime}\right) \cdot \boldsymbol{\xi}\left(s^{\prime}\right)\right]^{2}\right|_{j=\boldsymbol{h}=0}
\end{aligned}
$$

from which we clearly see that after performing the derivatives with respect to $\boldsymbol{j}$ and $\boldsymbol{h}$ and setting them zero, $w_{v \rightarrow n,}^{(0,3)}\left(t_{f}, \boldsymbol{\xi}\right]$ is odd in $\boldsymbol{\xi}$. Therefore, we conclude $w_{v \rightarrow n}^{(0,3)}\left(t_{f}\right) \equiv\left\langle\left\langle w_{v \rightarrow n}^{(0,3)}\left(t_{f}, \boldsymbol{\xi}\right]\right\rangle=0\right.$, that is, no contribution to the energy flow between two oscillators from the $P_{v \rightarrow n}^{(1,3)}\left(t_{f}\right)$ component.

Now we move on to the $\eta=4$ case. All we need to do is find the right hand side,

$$
w_{v \rightarrow n}^{(0,4)}\left(t_{f}, \boldsymbol{\xi}\right]=-\left.\sum_{k l m} \kappa_{k l m} \frac{\partial \delta^{3} \exp \left\{i \overline{\mathscr{S}}_{\xi}^{(0)}[\boldsymbol{j}, \boldsymbol{h})\right\}}{\partial h_{n} \delta j_{k}\left(t_{f}\right) \delta j_{l}\left(t_{f}\right) \delta j_{m}\left(t_{f}\right)}\right|_{j=\boldsymbol{h}=0} .
$$

One can see that only the fourth order in the Taylor expansion of $\exp \left\{i \overline{\mathscr{S}}_{\xi}^{(0)}[\boldsymbol{j}, \boldsymbol{h})\right\}$ will give a non-zero contribution since it contains the term $h \cdot j^{3}$. Carrying out this procedure yields,

$$
\begin{aligned}
& \left.\frac{\partial \delta^{3} \exp \left\{i \overline{\mathscr{S}}_{\xi}^{(0)}[\boldsymbol{j}, \boldsymbol{h})\right\}}{\partial h_{n} \delta j_{k}\left(t_{f}\right) \delta j_{l}\left(t_{f}\right) \delta j_{m}\left(t_{f}\right)}\right|_{\boldsymbol{j}=\boldsymbol{h}=0} \\
= & \left.\sum_{o p q r} \int \cdots \int_{0}^{t_{f}} d s d s_{1} d s_{2} d s_{3}\left[\dot{\boldsymbol{D}}_{2}\left(t_{f}-s\right)\right]_{n o} \cdot \boldsymbol{\xi}(s)\right]_{o}\left[\mathfrak{D}\left(t_{f}-s_{1}\right)\right]_{k p} \boldsymbol{\xi}_{p}\left(s_{1}\right)\left[\mathfrak{D}\left(t_{f}-s_{2}\right)\right]_{l q} \boldsymbol{\xi}_{q}\left(s_{2}\right)\left[\mathfrak{D}\left(t_{f}-s_{3}\right)\right]_{m r} \boldsymbol{\xi}_{r}\left(s_{3}\right) .
\end{aligned}
$$

We introduce a shorthand notation

$$
\Lambda_{k l m}^{n}\left(t_{f}\right) \equiv\left\langle\left.\left\langle\frac{\partial \delta^{3} \exp \left\{i \overline{\mathscr{S}}_{\xi}^{(0)}[\boldsymbol{j}, \boldsymbol{h})\right\}}{\partial h_{n} \delta j_{k}\left(t_{f}\right) \delta j_{l}\left(t_{f}\right) \delta j_{m}\left(t_{f}\right)}\right\rangle\right|_{\boldsymbol{j}=\boldsymbol{h}=0} .\right.
$$

Making change of variables $t_{f}-s \rightarrow s, t_{f}-s_{i} \rightarrow s_{i}$ and employing the trick given in (73), we obtain

$$
\begin{aligned}
& \Lambda_{k l m}^{n}\left(t_{f}\right)=\int \cdots \int_{-\infty}^{t_{f}} d s d s_{1} d s_{2} d s_{3}\{[\left.\mathfrak{D}(s) \boldsymbol{G}_{H}\left(s_{1}-s\right) \mathfrak{D}\left(s_{1}\right)\right]_{n k}[ \\
&+\left[\mathfrak{D}\left(s_{2}\right) \boldsymbol{G}_{H}\left(s_{3}-s_{2}\right) \mathfrak{D}\left(s_{3}\right)\right]_{l m} \\
&\left.+\boldsymbol{G}_{H}\left(s_{2}-s\right) \mathfrak{D}\left(s_{2}\right)\right]_{n l}\left[\mathfrak{D}\left(s_{1}\right) \boldsymbol{G}_{H}\left(s_{3}-s_{1}\right) \mathfrak{D}\left(s_{3}\right)\right]_{k m} \\
&\left.+\left[\dot{\mathfrak{D}}(s) \boldsymbol{G}_{H}\left(s_{3}-s\right) \mathfrak{D}\left(s_{3}\right)\right]_{n m}\left[\mathfrak{D}\left(s_{1}\right) \boldsymbol{G}_{H}\left(s_{2}-s_{1}\right) \mathfrak{D}\left(s_{2}\right)\right]_{k l}\right\} .
\end{aligned}
$$




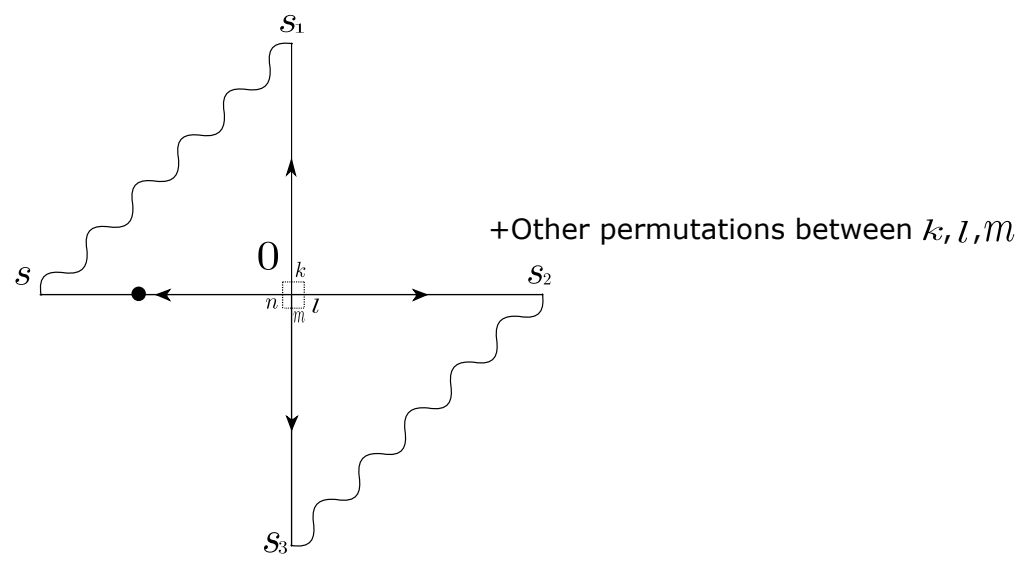

Figure 5: Time-domain diagrammatic representation of $\Lambda_{k l m}^{n}(\infty)$

Since from (107) for $\eta=4$, we know that the coefficients $\kappa_{k l m}$ will be determined by the binomial expansion of the form $\left[\delta / \delta j_{n}\left(t_{f}\right)-\delta / \delta j_{v}\left(t_{f}\right)\right]^{3}$, we thus find

$$
w_{v \rightarrow n, 0}^{(0,4)}(\infty)=-\sum_{k l m} \kappa_{k l m} \Lambda_{k l m}^{n}(\infty)=-\left[\Lambda_{n n n}^{n}(\infty)-3 \Lambda_{n n v}^{n}(\infty)+3 \Lambda_{n v v}^{n}(\infty)-\Lambda_{v v v}^{n}(\infty)\right],
$$

after the motion is fully relaxed.

Now we have calculated all the first-order corrections (78), (79) of the rates of the energy exchange between each oscillator and its private bath, as well as the correction (97), (113) of the energy flow between the neighboring oscillators. We have noted that 1) the $\alpha$-FPUT nonlinearity does not contribute to the quantities of our interest, and 2) these first-order corrections all become time-independent on a time scale much greater than the relaxation time. Thus it seems to imply a steady state for the current configuration. We will address this feature in Sec. 6

We conclude this section by highlighting that the first-order nonlinear corrections to the rate of the energy exchange between the oscillators consist of two distinct components: (a) the first-order correction of the current $P_{v \rightarrow n}^{(2)}$ corresponding to the intra-oscillator quadratic coupling, which can be induced by either cubic $\alpha$-FPUT or $\beta$-FPUT and KG quartic interaction in the chain Hamiltonian. (b) the the first-order correction of the current $P_{\nu \rightarrow n}^{(\eta)}(\eta \geq 3)$ corresponding to the intra-oscillator cubic $\alpha$-FPUT or quartic $\beta$-FPUT coupling. Obviously, implied by (a) and (b), the KG nonlinearity is self-interaction at each site and does not couple two different oscillators. So it only induces a correction to $P_{v \rightarrow n}^{(2)}$ and does not generate the current $P_{v \rightarrow n}^{(4)}$.

\section{Diagrammatic representations}

From now on, we will discuss the steady-state energy and will implicitly assume $t_{f}=\infty$. Therefore we suppress all the time dependence in all the relevant energy currents, which are given by Eqs. $(51,53,56,74,77,95,96,112)$ respectively. Although they may look formidable, all these energy currents admit time-domain diagrammatic representations, which can be seen directly from their respective analytic expressions through definite rules. Furthermore, one can easily convert the time-domain diagrams to the frequency domain diagrams, which allows one to obtain the Fourier transform of these energy currents in an economical way. Finally, these diagrams provide an intuitive understanding of the energy exchange between the baths and the oscillators and among the oscillators.

\subsection{Properties of causal propagator $\tilde{\mathfrak{D}}(\omega)$}

Before moving to the diagrammatic representation, let us discuss some useful properties of the Fourier transform the the causal propagator $\mathfrak{D}(s)$, which will be used later. According to Eq. (C.12), the Laplace transform of $\boldsymbol{D}_{2}(s)$, i.e., $\boldsymbol{D}_{2}(\mathfrak{s})$ is symmetric and so is $\boldsymbol{D}_{2}(s)$. Here one should not be confused with the variable in the Laplace domain 

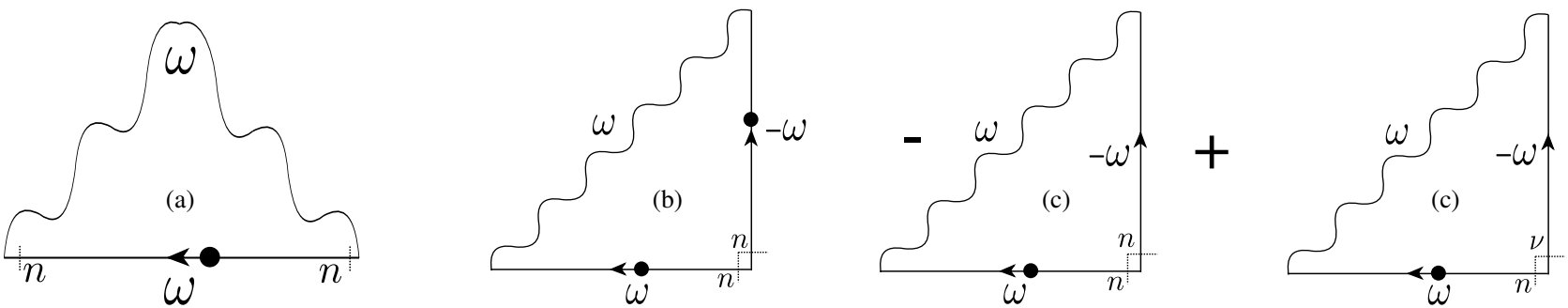

Figure 6: Frequency-domain diagrammatic representation of the zeroth-order energy currents including Figs. (a) $P_{\xi_{n}}(\infty)(\mathrm{b}) p_{n}^{2(0)}(\infty)(\mathrm{c}) w_{n, 2}^{v}(\infty)$. As we show Sec. 5.1, the first digram in (c) actually vanishes.

denoted as $\mathfrak{s}$ and the variable in the time domain denoted as $s$. According to the relation between Fourier and Laplace transform, one can readily find

$$
\tilde{\mathfrak{D}}(\omega)=\boldsymbol{D}_{2}(\mathfrak{s}=i \omega)=\left[-\omega^{2}+2 i \gamma \omega+\mathbf{\Omega}_{R}^{2}\right]^{-1},
$$

where $\boldsymbol{\Omega}_{R}^{2}$ defined in Eq. (C.7) is the renormalized version of Eq. (44). From Eq. (114), we know

$$
\begin{gathered}
{[\tilde{\mathfrak{D}}(\omega)]_{12}=[\tilde{\mathfrak{D}}(\omega)]_{21},} \\
{[\tilde{\mathfrak{D}}(\omega)]_{11}=[\tilde{\mathfrak{D}}(\omega)]_{22},} \\
\left(-\omega^{2}+2 i \gamma \omega+\omega_{R 0}^{2}+\lambda_{2}\right)[\tilde{\mathfrak{D}}(\omega)]_{n n}-\lambda_{2}[\tilde{\mathfrak{D}}(\omega)]_{n v}=1, \\
\left(-\omega^{2}+2 i \gamma \omega+\omega_{R 0}^{2}+\lambda_{2}\right)[\tilde{\mathfrak{D}}(\omega)]_{n v}-\lambda_{2}[\tilde{\mathfrak{D}}(\omega)]_{n n}=0 .
\end{gathered}
$$

where $v \neq n$. Furthermore, since $\mathfrak{D}(s)$ is real, one can easily conclude

$$
\tilde{\mathfrak{D}}^{*}(\omega)=\tilde{\mathfrak{D}}(-\omega)
$$

or equivalently $\operatorname{Re} \tilde{\mathfrak{D}}(\omega)$ is even in $\omega$ while $\operatorname{Im} \tilde{\mathfrak{D}}(\omega)$ is odd in $\omega$.

\subsection{Feynman diagrams in the time domain}

The following rules can be used in order convert the analytic expressions typically in Eqs. (51, 52, 56,74, 77, 95, 96, 112 ) into diagrams shown in Figs. 2-5.

1. $s_{1} \rightarrow s_{2}$ denotes the causal Green's function of the chain $\mathfrak{D}\left(s_{2}-s_{1}\right)$.

2. The bullet denotes the time derivative with respect to the argument of the chain's causal Green's function. Therefore $s_{1} \longrightarrow \bullet s_{2}$ or $s_{1} \longrightarrow s_{2}$ is the time derivative the causal Green's function of the chain, i.e., $\mathfrak{D}\left(s_{2}-s_{1}\right)$, where the overhead dot denotes the derivative with respect to the argument of $\mathfrak{D}\left(s_{2}-s_{1}\right)$. The order that the arrow and the bullet appears does not matter.

3. $s_{1} \mathrm{unn}_{2}$ denotes the Hadamard Green's function of the field $\boldsymbol{G}_{H}\left(s_{2}-s_{1}\right)$ or $\boldsymbol{G}_{H}\left(s_{1}-s_{2}\right)$. There is no arrows placed on the wavy line since $\boldsymbol{G}_{H}$ is symmetric in $s_{1}$ and $s_{2}$.

4. All the vortices such as $s, s^{\prime}, s_{i}$, except the origin, must be integrated out, with integration limit goes from $-\infty$ to $+\infty$ (since we implicitly assume $t_{f}=\infty$ ).

5. The indices of matrix elements associated with the propagators are indicated by the letters beside the short line segments.

Conversely, with above rules, one can recover Eqs. (51, 52, 56,74, 77, 95, 96, 112 ) from Figs. 2-5. 

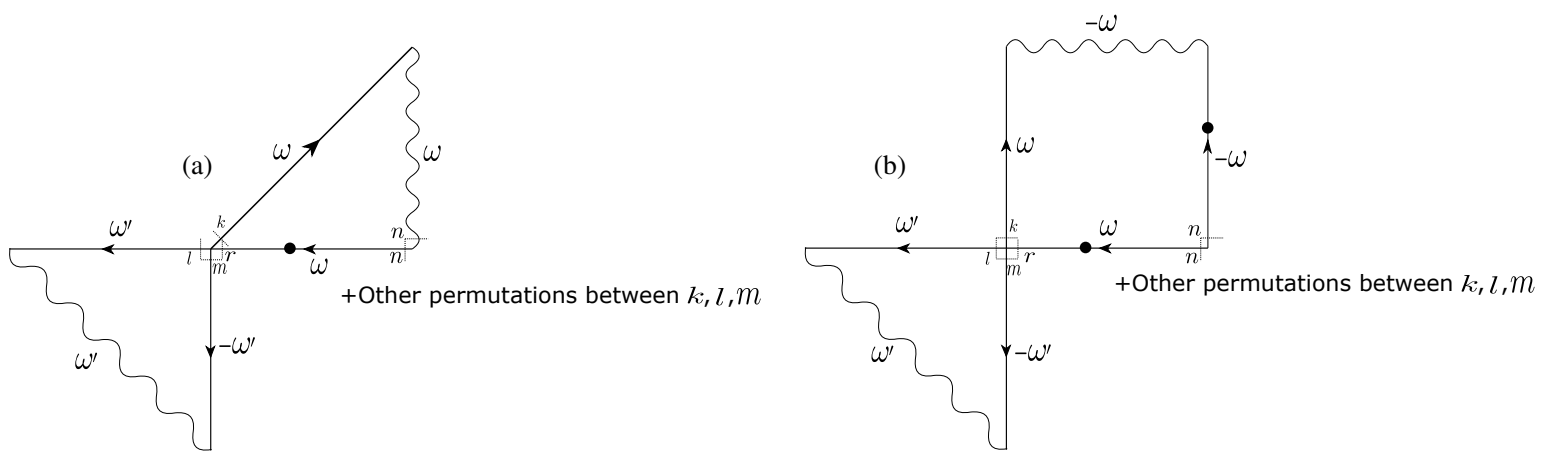

Figure 7: Frequency-domain diagrammatic representations of $\Gamma_{k l m}^{n r}(\infty)$ and $\tilde{\Gamma}_{k l m}^{n r}(\infty)$ are shown in (a) and (b) respectively. Note that the factor of 2 in Eq. (77) is not shown in (b).

\subsection{Feynman diagrams in the frequency domain}

In standard quantum field theory, the momentum-space Feynman-diagrams are far more important and easier to work with than their position-space counter parts. Similarly, here the diagrams in the frequency domain are more useful in proving the NESS. One can convert the time-domain diagrams shown in Figs. 2-5 according to the following rules:

1. Removing all the time arguments but keeping the indices of the matrix element from the time-domain diagrams

2. Associated frequencies (energies) with for both the causal and Hadamard propagators such that the energy is conserved at all the vortices.

3. When imposing energy conservation at each vortex, the arrows in the causal propagator denotes the direction of energy flow. The direction of the energy flow in the Hadamard propagator is omitted since it can be easily inferred by the frequency associated with it and the energy conservation.

After implementing above procedures, all the corresponding frequency-domain diagrams are shown in Figs. (6)-(9). One can directly read off the Fourier transforms of Eqs. (51, 52, 56,74, 77, 95, 96, 112 ) from Figs. (6)-(9), according to the following rules:

1. $\rightarrow$ denotes $\tilde{D}( \pm \omega)$, the Fourier transform of the causal propagator $\mathfrak{D}(s)$, where the sign depends on the frequency associated with the propagator.

2 . $\rightarrow \bullet$ or $\rightarrow$ denotes $( \pm i \omega) \tilde{D}( \pm \omega)$. Again the sign depends on the sign of the frequency associated with the propagator.

3. mun denotes $\tilde{\boldsymbol{G}}_{H}( \pm \omega)$, the Fourier transform of $\boldsymbol{G}_{H}(s)$, where the sign depends on the assigned frequency.

4. In the end, the independent frequencies must be integrated out with the measure $\int d \omega /(2 \pi)$. Note that the number of independent frequencies are equal to the number of loops in the diagrams.

According to above rules, one can easily read off the the expression of all the zeroth order correction from Fig. 6

$$
\begin{gathered}
P_{\xi_{n}}^{(0)}=\frac{1}{2 \pi} \int_{-\infty}^{\infty}(i \omega)\left[\tilde{\mathfrak{D}}(\omega) \tilde{\boldsymbol{G}}_{H}(\omega)\right]_{n n} d \omega, \\
p_{n}^{2(0)}=\frac{1}{2 \pi} \int_{-\infty}^{\infty} \omega^{2}\left[\tilde{\mathfrak{D}}(\omega) \tilde{\boldsymbol{G}}_{H}(\omega) \tilde{\mathfrak{D}}(-\omega)\right]_{n n} d \omega, \\
\left.w_{v \rightarrow n}^{(0,2)}=-\frac{1}{2 \pi} \int_{-\infty}^{\infty} i \omega\left(\left[\tilde{\mathfrak{D}}(\omega) \tilde{\boldsymbol{G}}_{H}(\omega) \tilde{\mathfrak{D}}(-\omega)\right]_{n n}-\tilde{\mathfrak{D}}(\omega) \tilde{\boldsymbol{G}}_{H}(\omega) \tilde{\mathfrak{D}}(-\omega)\right]_{n v}\right) d \omega .
\end{gathered}
$$

With Eq. (119), one can easily show that

$$
\int_{-\infty}^{\infty} i \omega\left[\tilde{\mathfrak{D}}(\omega) \tilde{\boldsymbol{G}}_{H}(\omega) \tilde{\mathfrak{D}}(-\omega)\right]_{n n}=\sum_{k=n, v} \int_{-\infty}^{\infty} i \omega\left|[\tilde{\mathfrak{D}}(\omega)]_{n k}\right|^{2}\left[\tilde{\boldsymbol{G}}_{H}(\omega)\right]_{k k}=0,
$$


since the integrand is an odd function of $\omega$. Eq. (122) becomes

$$
w_{\nu \rightarrow n}^{(0,2)}=\frac{1}{2 \pi} \int_{-\infty}^{\infty} i \omega\left[\tilde{\mathfrak{D}}(\omega) \tilde{\boldsymbol{G}}_{H}(\omega) \tilde{\mathfrak{D}}(-\omega)\right]_{n \nu} d \omega .
$$

Equivalently, Eq. (123) justifies that the first diagram in Fig. 2(c) or Fig.6(c) vanishes. Similarly, from Fig. 7, one can easily obtain the frequency-domain representation for $\Gamma_{k l m}^{n r}$ and $\tilde{\Gamma}_{k l m}^{n r}$

$$
\begin{aligned}
\Gamma_{k l m}^{n r} & =\frac{1}{(2 \pi)^{2}} \iint d \omega d \omega^{\prime}(i \omega)[\tilde{\mathfrak{D}}(\omega)]_{n r}\left\{\left[\tilde{\boldsymbol{G}}_{H}(\omega) \tilde{\mathfrak{D}}(\omega)\right]_{n k}\left[\tilde{\mathfrak{D}}\left(\omega^{\prime}\right) \tilde{\boldsymbol{G}}_{H}\left(\omega^{\prime}\right) \tilde{\mathfrak{D}}\left(-\omega^{\prime}\right)\right]_{l m}\right. \\
& +\left[\tilde{\boldsymbol{G}}_{H}(\omega) \tilde{\mathfrak{D}}(\omega)\right]_{n l}\left[\tilde{\mathfrak{D}}\left(\omega^{\prime}\right) \tilde{\boldsymbol{G}}_{H}\left(\omega^{\prime}\right) \tilde{\mathfrak{D}}\left(-\omega^{\prime}\right)\right]_{k m} \\
& \left.+\left[\tilde{\boldsymbol{G}}_{H}(\omega) \tilde{\mathfrak{D}}(\omega)\right]_{n m}\left[\tilde{\mathfrak{D}}\left(\omega^{\prime}\right) \tilde{\boldsymbol{G}}_{H}\left(\omega^{\prime}\right) \tilde{\mathfrak{D}}\left(-\omega^{\prime}\right)\right]_{k l}\right\} \\
\tilde{\Gamma}_{k l m}^{n r}= & \frac{2}{(2 \pi)^{2}} \iint d \omega d \omega^{\prime} \omega^{2}[\tilde{\mathfrak{D}}(\omega)]_{n r}\left\{\left[\tilde{\mathfrak{D}}(-\omega) \tilde{\boldsymbol{G}}_{H}(-\omega) \tilde{\mathfrak{D}}(\omega)\right]_{n k}\left[\tilde{\mathfrak{D}}\left(\omega^{\prime}\right) \tilde{\boldsymbol{G}}_{H}\left(\omega^{\prime}\right) \tilde{\mathfrak{D}}\left(-\omega^{\prime}\right)\right]_{l m}\right. \\
+ & {\left[\tilde{\mathfrak{D}}(-\omega) \tilde{\boldsymbol{G}}_{H}(-\omega) \tilde{\mathfrak{D}}(\omega)\right]_{n l}\left[\tilde{\mathfrak{D}}\left(\omega^{\prime}\right) \tilde{\boldsymbol{G}}_{H}\left(\omega^{\prime}\right) \tilde{\mathfrak{D}}\left(-\omega^{\prime}\right)\right]_{k m} } \\
& \left.+\left[\tilde{\mathfrak{D}}(-\omega) \tilde{\boldsymbol{G}}_{H}(-\omega) \tilde{\mathfrak{D}}(\omega)\right]_{n m}\left[\tilde{\mathfrak{D}}\left(\omega^{\prime}\right) \tilde{\boldsymbol{G}}_{H}\left(\omega^{\prime}\right) \tilde{\mathfrak{D}}\left(-\omega^{\prime}\right)\right]_{k l}\right\} .
\end{aligned}
$$

Finally, the frequency-domain diagrammatic representations for Eqs. $(95,96,112)$ are shown in Figs. 8 and 9 . From these diagrams, one can easily find

$$
\begin{aligned}
\Upsilon_{v k l m}^{n r} & =\frac{1}{(2 \pi)^{2}} \iint d \omega d \omega^{\prime}(i \omega)[\tilde{\mathfrak{D}}(\omega)]_{n r}\left\{\left[\tilde{\mathfrak{D}}(-\omega) \tilde{\boldsymbol{G}}_{H}(-\omega) \tilde{\mathfrak{D}}(\omega)\right]_{v k}\left[\tilde{\mathfrak{D}}\left(\omega^{\prime}\right) \tilde{\boldsymbol{G}}_{H}\left(\omega^{\prime}\right) \tilde{\mathfrak{D}}\left(-\omega^{\prime}\right)\right]_{l m}\right. \\
+ & {\left[\tilde{\mathfrak{D}}(-\omega) \tilde{\boldsymbol{G}}_{H}(-\omega) \tilde{\mathfrak{D}}(\omega)\right]_{v l}\left[\tilde{\mathfrak{D}}\left(\omega^{\prime}\right) \tilde{\boldsymbol{G}}_{H}\left(\omega^{\prime}\right) \tilde{\mathfrak{D}}\left(-\omega^{\prime}\right)\right]_{k m} } \\
+ & {\left.\left[\tilde{\mathfrak{D}}(-\omega) \tilde{\boldsymbol{G}}_{H}(-\omega) \tilde{\mathfrak{D}}(\omega)\right]_{v m}\left[\tilde{\mathfrak{D}}\left(\omega^{\prime}\right) \tilde{\boldsymbol{G}}_{H}\left(\omega^{\prime}\right) \tilde{\mathfrak{D}}\left(-\omega^{\prime}\right)\right]_{k l}\right\} } \\
\tilde{\Upsilon}_{v k l m}^{n r}= & \frac{1}{(2 \pi)^{2}} \iint d \omega d \omega^{\prime}(-i \omega)[\tilde{\mathfrak{D}}(\omega)]_{v r}\left\{\left[\tilde{\mathfrak{D}}(-\omega) \tilde{\boldsymbol{G}}_{H}(-\omega) \tilde{\mathfrak{D}}(\omega)\right]_{n k}\left[\tilde{\mathfrak{D}}\left(\omega^{\prime}\right) \tilde{\boldsymbol{G}}_{H}\left(\omega^{\prime}\right) \tilde{\mathfrak{D}}\left(-\omega^{\prime}\right)\right]_{l m}\right. \\
+ & {\left[\tilde{\mathfrak{D}}(-\omega) \tilde{\boldsymbol{G}}_{H}(-\omega) \tilde{\mathfrak{D}}(\omega)\right]_{n l}\left[\tilde{\mathfrak{D}}\left(\omega^{\prime}\right) \tilde{\boldsymbol{G}}_{H}\left(\omega^{\prime}\right) \tilde{\mathfrak{D}}\left(-\omega^{\prime}\right)\right]_{k m} } \\
+ & {\left.\left[\tilde{\mathfrak{D}}(-\omega) \tilde{\boldsymbol{G}}_{H}(-\omega) \tilde{\mathfrak{D}}(\omega)\right]_{n m}\left[\tilde{\mathfrak{D}}\left(\omega^{\prime}\right) \tilde{\boldsymbol{G}}_{H}\left(\omega^{\prime}\right) \tilde{\mathfrak{D}}\left(-\omega^{\prime}\right)\right]_{k l}\right\} } \\
& \Lambda_{k l m}^{n}=\frac{1}{(2 \pi)^{2}} \iint d \omega d \omega^{\prime}(i \omega)\left\{\left[\tilde{\mathfrak{D}}(\omega) \tilde{\boldsymbol{G}}_{H}(\omega) \tilde{\mathfrak{D}}(-\omega)\right]_{n k}\left[\tilde{\mathfrak{D}}\left(\omega^{\prime}\right) \tilde{\boldsymbol{G}}_{H}\left(\omega^{\prime}\right) \tilde{\mathfrak{D}}\left(-\omega^{\prime}\right)\right]_{l m}\right. \\
& +\left[\tilde{\mathfrak{D}}(\omega) \tilde{\boldsymbol{G}}_{H}(\omega) \tilde{\mathfrak{D}}(-\omega)\right]_{n l}\left[\tilde{\mathfrak{D}}\left(\omega^{\prime}\right) \tilde{\boldsymbol{G}}_{H}\left(\omega^{\prime}\right) \tilde{\mathfrak{D}}\left(-\omega^{\prime}\right)\right]_{k m} \\
& \left.+\left[\tilde{\mathfrak{D}}(\omega) \tilde{\boldsymbol{G}}_{H}(\omega) \tilde{\mathfrak{D}}(-\omega)\right]_{n m}\left[\tilde{\mathfrak{D}}\left(\omega^{\prime}\right) \tilde{\boldsymbol{G}}_{H}\left(\omega^{\prime}\right) \tilde{\mathfrak{D}}\left(-\omega^{\prime}\right)\right]_{k l}\right\} .
\end{aligned}
$$

One can check that Eqs. (120-129) are indeed Fourier transform of Eqs. (51, 52, 56,74, 77, 95, 96, 112 ) respectively, with the Fourier transform defined as $f(s)=1 /(2 \pi) \int_{-\infty}^{\infty} \tilde{f}(\omega) e^{i \omega s} d \omega$. From Fig. 8 or explicit expressions $(127,128)$, one can easily observe,

$$
\Upsilon_{n k l m}^{n r}=-\tilde{\Upsilon}_{n k l m}^{n r},
$$

which simplifies Eq. (97) as

$$
w_{v \rightarrow n}^{(1,2)}=\sum_{k l m r} \mu_{k l m r}\left[\Upsilon_{v k l m}^{n r}+\tilde{\Upsilon}_{v k l m}^{n r}\right]
$$



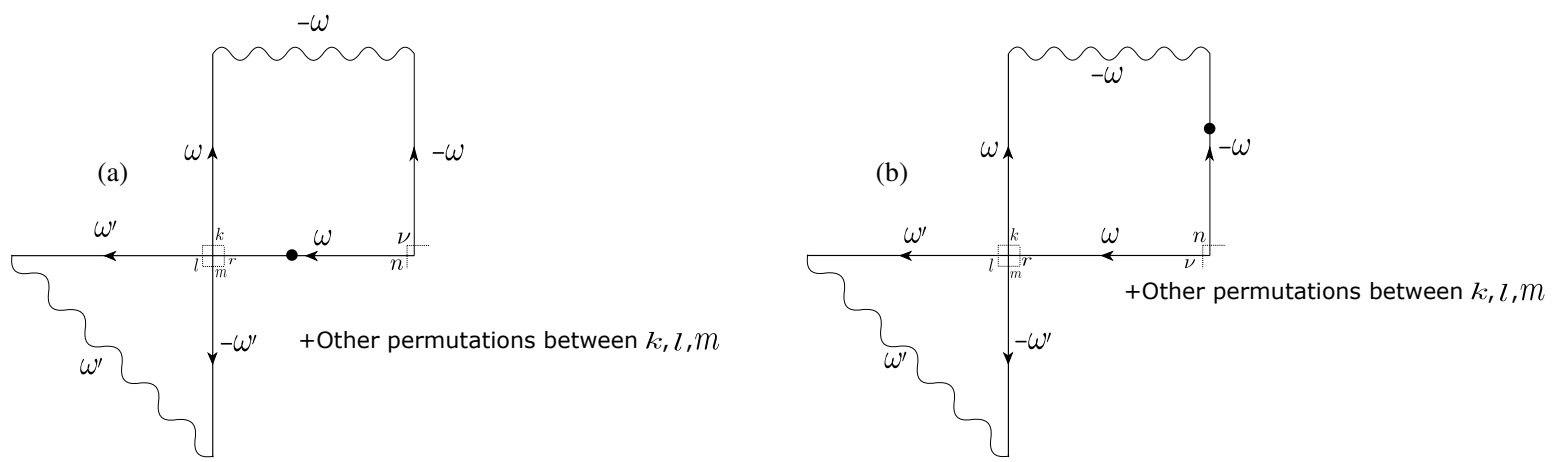

Figure 8: Frequency-domain diagrammatic representation of (a) $\Upsilon_{k l m}^{n r}(\infty)$ and (b) $\tilde{\Upsilon}_{v k l m}^{n r}(\infty)$

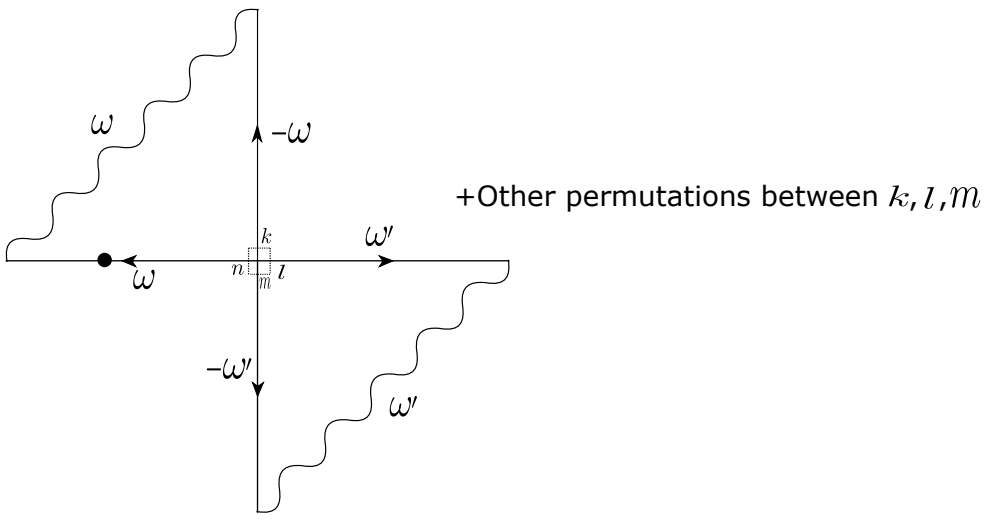

Figure 9: Frequency-domain diagrammatic representation of $\Lambda_{k l m}^{n}(\infty)$. According to Eqs. $(123,129), \Lambda_{n n n}^{n}(\infty)=0$.

\section{The non-equilibrium steady state (NESS)}

For the configuration we are interested, if the NESS exists, then we expect when the NESS is reached we will have a steady, time-independent energy current along the chain. Thus, in principle, in order to demonstrate the existence of the NESS, we would like to show for the configuration in consideration that in the late time limit $t_{f} \rightarrow \infty$, the time rate of the internal energy of each oscillator vanishes. In other words, we will show that the ensemble average of Eq. (22) vanishes in the late time limit $t_{f} \rightarrow \infty$, such that

$$
P_{\xi_{n}}+P_{\gamma_{n}}+P_{n-1 \rightarrow n}+P_{n+1 \rightarrow n}=0
$$

for each $n$

However, we will discuss a particular case, for the proof of concept, that involves only two oscillators in contact with their own private baths. We will show Eq. (132) holds in the late time limit, up to the first order. This is our main result. We start with the zeroth-order case, which has been shown to hold for a chain made of any number of linear oscillators, strung together by bilinear coupling [17], that is, the quadratic coupling discussed in this paper. Nevertheless, For sake of completeness, we will still demonstrate (132) for the zeroth-order contributions based the results derived by our approach in Sec. 3. Then we further proceed to the first-order corrections.

\subsection{NESS at the zeroth order}

From (120), (121), and (124), we use the property that 1) $\tilde{\boldsymbol{G}}_{H}(\omega)$ is diagonal, and 2) $\operatorname{Im} \tilde{\mathfrak{D}}(\omega)$ is odd in $\omega$, we can write them as

$$
\begin{gathered}
P_{\xi_{n}}^{(0)}=-\int_{-\infty}^{\infty} \frac{d \omega}{2 \pi} \omega \operatorname{Im}[\tilde{\mathfrak{D}}(\omega)]_{n n}\left[\tilde{\boldsymbol{G}}_{H}(\omega)\right]_{n n}, \\
P_{\gamma_{n}}^{(0)}=-2 \gamma \sum_{k} \int_{-\infty}^{\infty} \frac{d \omega}{2 \pi} \omega^{2}\left|[\tilde{\mathfrak{D}}(\omega)]_{n k}\right|^{2}\left[\tilde{\boldsymbol{G}}_{H}(\omega)\right]_{k k},
\end{gathered}
$$




$$
P_{v \rightarrow n}^{(0,2)}=-\lambda_{2} \sum_{k} \int_{-\infty}^{\infty} \frac{d \omega}{2 \pi} \omega \operatorname{Im}\left\{[\tilde{\mathfrak{D}}(\omega)]_{n k}\left[\tilde{\mathfrak{D}}^{*}(\omega)\right]_{v k}\right\}\left[\tilde{\boldsymbol{G}}_{H}(\omega)\right]_{k k}
$$

So we obtain

$$
\begin{aligned}
& P_{\xi_{n}}^{(0)}+P_{\gamma_{n}}^{(0)}+P_{v \rightarrow n}^{(0,2)} \\
= & -\frac{1}{2 \pi} \int_{-\infty}^{\infty}\left\{\omega \operatorname{Im}[\tilde{\mathfrak{D}}(\omega)]_{n n}+2 \gamma \omega^{2}\left|[\tilde{\mathfrak{D}}(\omega)]_{n n}\right|^{2}+\lambda_{2} \omega \operatorname{Im}\left([\tilde{\mathfrak{D}}(\omega)]_{n n}[\tilde{\mathfrak{D}}(\omega)]_{v n}^{*}\right)\right\}\left[\tilde{\boldsymbol{G}}_{H}(\omega)\right]_{n n} \\
- & \frac{1}{2 \pi} \int_{-\infty}^{\infty}\left\{2 \gamma \omega^{2}\left|[\tilde{\mathfrak{D}}(\omega)]_{n v}\right|^{2}+\lambda_{2} \omega \operatorname{Im}\left([\tilde{\mathfrak{D}}(\omega)]_{n v}[\tilde{\mathfrak{D}}(\omega)]_{v v}^{*}\right)\right\}\left[\tilde{\boldsymbol{G}}_{H}(\omega)\right]_{v v} .
\end{aligned}
$$

Now by replacing eliminating $[\tilde{\mathfrak{D}}(\omega)]_{v n}^{*}$ through Eqs. $(115,117)$, one can easily show that

$$
\omega \operatorname{Im}[\tilde{\mathfrak{D}}(\omega)]_{n n}+2 \gamma \omega^{2}\left|[\tilde{\mathfrak{D}}(\omega)]_{n n}\right|^{2}+\lambda_{2} \omega \operatorname{Im}\left([\tilde{\mathfrak{D}}(\omega)]_{n n}[\tilde{\mathfrak{D}}(\omega)]_{v n}^{*}\right)=0 .
$$

Similarly, by eliminating $[\tilde{\mathfrak{D}}(\omega)]_{v v}^{*}$ through Eqs. $(116,118)$, one can easily prove

$$
2 \gamma \omega^{2}\left|[\tilde{\mathfrak{D}}(\omega)]_{n v}\right|^{2}+\lambda_{2} \omega \operatorname{Im}\left([\tilde{\mathfrak{D}}(\omega)]_{n v}[\tilde{\mathfrak{D}}(\omega)]_{v v}^{*}\right)=0 .
$$

Eqs. (137) and (138) together prove that for both $n=1,2$,

$$
P_{\xi_{n}}^{(0)}+P_{\gamma_{n}}^{(0)}+P_{\nu \rightarrow n}^{(0,2)}=0
$$

\subsection{NESS at the first order}

Here we address existence of the NESS at the first order of the nonlinear coupling constant. Since at this order, the contributions from the nonlinear couplings are additive, and the $\alpha$-FPUT coupling does not contribute, we consider only the KG- and the $\beta$-FPUT nonlinearity, and will show $P_{\xi_{n}}^{(1)}+P_{\gamma_{n}}^{(1)}+P_{v \rightarrow n}^{(1)}=0$ for $n=1$ and $n=2$

\subsubsection{KG nonlinearity}

When only KG nonlinearity is presented, we in fact have

$$
\begin{aligned}
& \sum_{k l m r} \sigma_{k l m r} r_{k}(s) q_{l}(s) q_{m}(s) q_{r}(s)=-\frac{\lambda_{\mathrm{KG}}}{4} \sum_{k=1}^{2} r_{k} q_{k}^{3}, \\
& \sum_{k l m r} \mu_{k l m r} r_{k}(s) r_{l}(s) r_{m}(s) q_{r}(s)=-\lambda_{\mathrm{KG}} \sum_{k=1}^{2} r_{k}^{3} q_{k} .
\end{aligned}
$$

Substitution of Eq. (141) into Eqs. (78), (79), yields

$$
P_{\xi_{n}}^{(1)}=-\lambda_{\mathrm{KG}} \sum_{k=1}^{2} \Gamma_{k k k}^{n k}, \quad \quad P_{\gamma_{n}}^{(1)}=2 \gamma \lambda_{\mathrm{KG}} \sum_{k=1}^{2} \tilde{\Gamma}_{k k k}^{n k},
$$

and from (33) and (97), we also obtain

$$
P_{v \rightarrow n}^{(1,2)}=-\lambda_{\mathrm{KG}} \lambda_{2} \sum_{k=1}^{2}\left(\Upsilon_{v k k k}^{n k}+\tilde{\Upsilon}_{v k k k}^{n k}\right)
$$

As a reminder, $P_{v \rightarrow n}^{(1,2)}$ is the first-order correction to the energy flow $P_{v \rightarrow n}^{(2)}$ due to the quadratic intra-oscillator coupling. Note that although both KG and $\beta$-FPUT are quartic interactions, the KG nonlinearity only involves self-interaction 

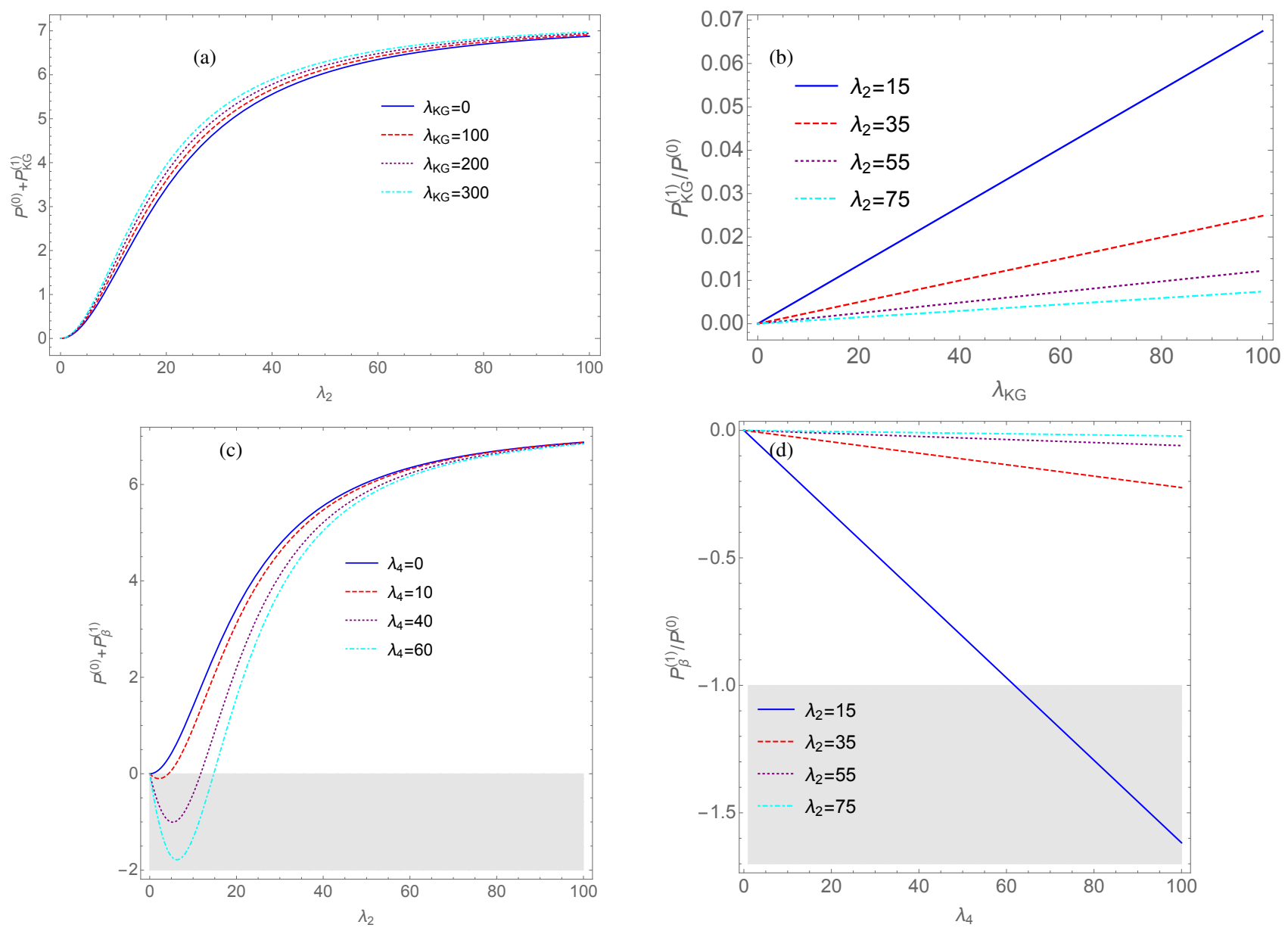

Figure 10: Energy current up to the first order across the two oscillators for (a) KG and (c) $\beta$-FPUT nonlinearities. The ratio between the first-order energy current and the zeroth for (b) KG and (d) $\beta$-FPUT nonlinearities. Value of parameters for all the figures: $\omega_{0}=10 ; \gamma=1$; the temperature of the hot bath is $T_{H}=\beta_{1}^{-1}=100$ and the temperature of the cold bath is $T_{C}=\beta_{2}^{-1}=0.002$. Note the first-order correction is positive for KG nonlinearity and negative $\beta$-FPUT nonlinearity. Therefore for $\beta$-FPUT when the ratio $\left|P_{\beta}^{(1)} / P^{(0)}\right| \geq 1$, as shown in the gray shaded region in Figs. $(\mathrm{c}, \mathrm{d})$, the energy current up to the first-order may become negative, meaning heat flow from the cold bath to hot bath. However, we note that this effect is spurious as whenever, $\left|P_{\beta}^{(1)} / P^{(0)}\right| \sim 1$, our perturbative calculation breaks down. 
at each site and therefore, unlike the $\beta$-FPUT interactions, does not induce $P_{v \rightarrow n}^{(4)}$. In Appendix E, we have shown the following the identities

$$
\begin{aligned}
-\Gamma_{n n n}^{n n}+2 \gamma \tilde{\Gamma}_{n n n}^{n n}-\lambda_{2}\left(\Upsilon_{v n n n}^{n n}+\tilde{\Upsilon}_{v n n n}^{n n}\right) & =0, \\
-\Gamma_{v v v}^{n v}+2 \gamma \tilde{\Gamma}_{v v v}^{n v}-\lambda_{2}\left(\Upsilon_{v v v v}^{n v}+\tilde{\Upsilon}_{v v v v}^{n v}\right) & =0 .
\end{aligned}
$$

With these identity, we see that

$$
P_{\xi_{n}}^{(1)}+P_{\gamma_{n}}^{(1)}+P_{v \rightarrow n}^{(1,2)}=0
$$

for both $n=1$ and $n=2$ in the presence of the KG-type nonlinearity.

\subsection{2. $\beta$-FPUT nonlinearity}

Next we move on to the $\beta$-FPUT nonlinearity. When only the $\beta$-FPUT nonlinearity is presented, we can show that terms on the righthand side of Eq. (41) becomes

$$
\begin{gathered}
\sum_{k l m r} \sigma_{k l m r} r_{k}(s) q_{l}(s) q_{m}(s) q_{r}(s)=-\frac{\lambda_{4}}{4}\left(q_{n}-q_{v}\right)\left(r_{n}-r_{v}\right)^{3}, \\
\sum_{k l m r} \mu_{k l m r} r_{k}(s) r_{l}(s) r_{m}(s) q_{r}(s)=-\lambda_{4}\left(q_{n}-q_{v}\right)\left(r_{n}-r_{v}\right)^{3},
\end{gathered}
$$

where the pairs $(n, v)$ could be either $(1,2)$ or $(2,1)$. Substituting Eq. (148) into Eqs. $(78,79,97)$, we obtain the first-order correction for the following energy fluxes

$$
P_{\xi_{n}}^{(1)}=-\lambda_{4} c_{n}(\Gamma), \quad P_{\gamma_{n}}^{(1)}=2 \gamma \lambda_{4} c_{n}(\tilde{\Gamma}), \quad P_{v \rightarrow n}^{(1,2)}=-\lambda_{2} \lambda_{4}\left[f_{v n}(\Upsilon)+f_{v n}(\tilde{\Upsilon})\right]
$$

where

$$
\begin{aligned}
c_{n}(\Gamma) & \equiv \Gamma_{n n n}^{n n}-3 \Gamma_{n n v}^{n n}+3 \Gamma_{n v v}^{n n}-\Gamma_{v v v}^{n n}-\Gamma_{n n n}^{n v}+3 \Gamma_{n n v}^{n v}-3 \Gamma_{n v v}^{n v}+\Gamma_{v v v}^{n v}, \\
f_{v n}(\Upsilon) & \equiv \Upsilon_{v n n n}^{n n}-3 \Upsilon_{v n n v}^{n n}+3 \Upsilon_{v n v v}^{n n}-\Upsilon_{v v v v}^{n n}-\Upsilon_{v n n n}^{n v}+3 \Upsilon_{v n n v}^{n v}-3 \Upsilon_{v n v v}^{n v}+\Upsilon_{v v v v}^{n v}
\end{aligned}
$$

Eqs. $(123,129)$ indicates that $\Lambda_{n n n}^{n}=0$. Thus, from Eqs. $(33,113)$, we find

$$
P_{v \rightarrow n}^{(1,4)}=\lambda_{4}\left[3 \Lambda_{n n v}^{n}-3 \Lambda_{n v v}^{n}+\Lambda_{v v v}^{n}\right]
$$

Here $P_{v \rightarrow n}^{(1,4)}$ is the first-order correction to the energy flow due to the quartic intra-oscillator coupling. In Appendix E, we show that

$$
\begin{aligned}
& -\Gamma_{k l m}^{n v}+2 \gamma \tilde{\Gamma}_{k l m}^{n v}-\lambda_{2}\left(\Upsilon_{v k l m}^{n v}+\tilde{\Upsilon}_{v k l m}^{n v}\right)=0, \\
& -\Gamma_{n k v}^{n n}+2 \gamma \tilde{\Gamma}_{n k v}^{n n}-\lambda_{2}\left(\Upsilon_{v n k v}^{n n}+\tilde{\Upsilon}_{v n k v}^{n n}\right)=\Lambda_{n k v}^{n}, \\
& -\Gamma_{v v v}^{n n}+2 \gamma \tilde{\Gamma}_{v v v}^{n n}-\lambda_{2}\left(\Upsilon_{v v v v}^{n n}+\tilde{\Upsilon}_{v v v v}^{n n}\right)=\Lambda_{v v v}^{n},
\end{aligned}
$$

where $k, l, m=n, v$. With Eqs. $(144,153-155)$, we readily obtain

$$
P_{\xi_{n}}^{(1)}+P_{\gamma_{n}}^{(1)}+P_{\nu \rightarrow n}^{(1,2)}+P_{v \rightarrow n}^{(1,4)}=0
$$

for $n=1,2$.

Together with $P_{v \rightarrow n}^{(1,4)}$, the net energy flow from site $v$ to site $n$ to the first order in the $\beta$-FPUT intra-oscillator coupling, we can define

$$
P_{\nu \rightarrow n}^{(\leq 1)}=P_{\nu \rightarrow n}^{(0,2)}+P_{\nu \rightarrow n}^{(1,2)}+P_{\nu \rightarrow n}^{(1,4)}
$$

representing the energy flow from site $v$ to site $n$, up to the first order. Note that the $P_{v \rightarrow n}^{(0,4)}=0$ according to the definition (33). We define

$$
P_{\mathrm{B}_{n}}^{(\leq 1)}=P_{\xi_{n}}^{(0)}+P_{\gamma_{n}}^{(0)}+P_{\xi_{n}}^{(1)}+P_{\gamma_{n}}^{(1)},
$$



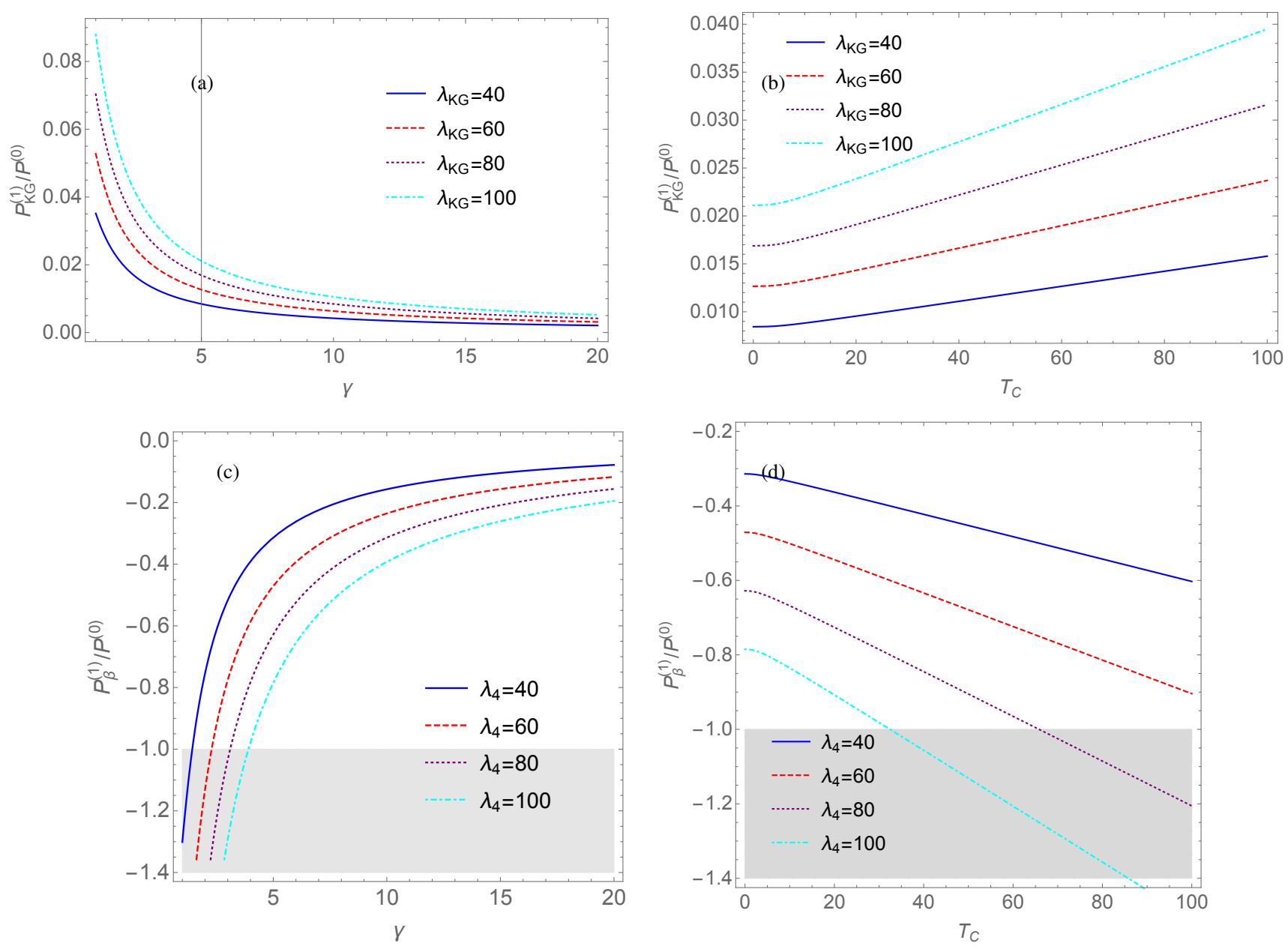

Figure 11: The role of coupling (damping) and the temperature bias in mediating the strength of (a, b) KG and (c, d) $\beta$-FPUT nonlinearities. Common values of the parameters for all figures: $\omega_{0}=\lambda_{2}=10$, the temperature of the hot bath is $T_{H}=\beta_{1}^{-1}=100$. For (a) and (c): the temperature of the cold bath is $T_{C}=\beta_{2}^{-1}=0.002$. For (b) and (d), $\gamma=5$. The gray shaded region shown in Figs. (c, d) corresponding to $\left|P_{\beta}^{(1)} / P^{(0)}\right| \geq 1$, where our perturbative calculation breaks down. Therefore, the seemingly heat flow from the cold bath to the hot bath is artificial. 
the net energy flow into oscillator $i$ from its private bath, up to the first order. Thus (156) becomes

$$
P_{\mathrm{B}_{n}}^{(\leq 1)}=-P_{\nu \rightarrow n}^{(\leq 1)}
$$

at late times. It means we have a steady energy flow through each oscillator. The direction of the flow will be determined by the temperatures of the private baths. As seen from [17, 62], at the zeroth-order, energy always flows from the higher temperature bath to the lower one. Here, even we consider its first-order correction due to nonlinear couplings, within the validity of the perturbative treatment, the corrections are required much smaller than the zerothorder contribution, and thus will not change the direction of the flow.

We conclude this section by noting that the zeroth-order heat fluxes, $P_{\xi_{n}}^{(0)}$ and $P_{\gamma_{n}}^{(0)}$ given in Eqs. $(133,134)$ diverge, which can be seen by counting the powers of $\tilde{\boldsymbol{G}}_{H}(\omega)$ and $\tilde{\mathfrak{D}}(\omega)$ at large $\omega$. Therefore, we need to perform regularization in order to evaluate them. We see that their regularized sum, according to the NESS condition at the zeroth order, equals to the finite $-P_{v \rightarrow n}^{(0,2)}$, which again can be seen by counting the powers of $\tilde{\boldsymbol{G}}_{H}(\omega)$ and $\tilde{\mathfrak{D}}(\omega)$ at large $\omega$. By similar analysis, one can readily find all the first-order corrections of the heat fluxes in the KG and $\beta$-FPUT model are all finite.

\section{Discussion and Conclusion}

Having established the NESS in the late time limit in the last section, we now show how our formal perturbative results presented in Sec. 3 and 4 naturally give a measure of the strength of nonlinearity for nonlinear open quantum systems with KG and $\beta$-FPUT type nonlinearity. This should provide insights for understanding and controlling the mesoscopic quantum heat transport. We denote the steady-state energy flows across the two oscillators at the zeroth order, the first-order for KG and $\beta$-FPUT types of nonlinearity as $P^{(0)}, P_{\mathrm{KG}}^{(1)}$ and $P_{\beta}^{(1)}$ respectively. Then the ratios $P_{\mathrm{KG}}^{(1)} / P^{(0)}$ and $P_{\beta}^{(1)} / P^{(0)}$, which characterize the strength of nonlinearity, can provide a measurement of how valid a perturbative calculations is, namely, as long as these ratios are much smaller than one.

As shown in Fig. 10, the ratios $P_{\mathrm{KG}}^{(1)} / P^{(0)}$ and $P_{\beta}^{(1)} / P^{(0)}$ become smaller with the increase of the quadratic intraoscillator coupling constant or the decrease of the quartic coupling constants $\lambda_{\mathrm{KG}}$ and $\lambda_{4}$. However, comparing Fig. 10 (a) with (c) or (b) with (d), we find two distinct features between the KG and $\beta$-FPUT nonlinearities: First, the KG nonlinearity is weaker than the $\beta$-FPUT nonlinearities in that under the same value of parameters $P_{\mathrm{KG}}^{(1)} / P^{(0)} \mathrm{smaller}$ than $P_{\beta}^{(1)} / P^{(0)}$. Secondly, the KG nonlinearity tends to strengthen the zeroth order heat flow since it induces a positive first-order correction while for the $\beta$-FPUT tends to weaken the zeroth order heat flow since it induces a negative firstorder correction. As shown in Figs. 10(c, d), for the gray shaded region, where $\left|P_{\beta}^{(1)} / P^{(0)}\right| \geq 1$, the net energy current up to the first-order becomes spuriously negative, implying heat flow from the cold bath to hot bath spontaneously. However, we should emphasize that this regime actually belongs to the one of strong nonlinearity, which lies beyond the reach of our perturbative calculation here. Nonetheless we see the general tendency in how nonlinear coupling may affect the energy transport along the chain. Hence our perturbative result, although not applicable in the regime of strong nonlinearity, may still provide some useful physical insights into this regime.

Fig. 11 illustrates how the nonlinear ratio $P^{(1)} / P^{(0)}$ changes when the coupling between the two oscillators and their respective private baths and the temperature bias are tuned. Fig. 11(a) and (c) indicate that for both types of nonlinearities, the stronger the coupling is, the less the transport deviates from the linear one. Similarly, by increasing the temperature bias between the two baths, one may also be able to bring the transport from strong nonlinear regime to weak nonlinear regime, as shown in Fig. 11(b) and (d).

On the contrary, as discussed in [62], the absorption refrigerator consisting of a network of linear oscillators and three-terminal baths does not produce any heat flow from the cold to the hot bath through the working bath when reaching NESS. Therefore, in order to reach efficient cooling, one must sufficiently tune the device away from the linear transport regime. The formal expression in Sec. 3 and 4 still applies, as long as the oscillator network is weakly nonlinear. One only needs to replace the causal propagator corresponding to two-oscillator two-bath configuration discussed here with the one corresponding to the three-oscillator three-bath configuration. Then our perturbative calculations here, although it cannot provide quantitatitive predictions about the transport in the strongly nonlinear regime, can still indicate the trend in how an interacting system enter the strongly nonlinear regime in order to generate significant cooling effects. 
In summary, we have provided a functional perturbative approach based on the stochastic generating functional which allows one to compute perturbatively the energy flux in the late time limit for a quantum anharmonic chain in the presence of weak nonlinearity. We considered the $\alpha$-FPUT, KG, and $\beta$-FPUT types of nonlinearities and gave the first order corrections to all the relevant energy fluxes to the first-order of nonlinear coupling constants. Up to this order, the contributions to the energy fluxes due to the initial state of the chain vanish in the late time limit. We found the first order corrections due to $\alpha$-FPUT nonlinearity vanish. For two coupled anharmonic oscillators that are coupled to their own private harmonic bath, the $\mathrm{KG}$ and $\beta$-FPUT nonlinearities lead to an NESS in the late time limit, up to the first-order of the nonlinear coupling constant. We conjectured that NESS exists in a chain with any of the $\alpha$-FPUT, $\mathrm{KG}$, and $\beta$-FPUT types of nonlinearities, to arbitrary orders of the respective nonlinear the coupling constant. From the fractional change of the energy current due to nonlinearity, its dependence on the oscillator-bath coupling and bath temperature bias can provide information about the range of validity of the perturbative analysis. This also reveals the tendency by which the energy currents can be modified by the nonlinearities. The expressions for the energy currents we found can be straightforwardly extended to an anharmonic chain consisting of arbitrary number of oscillators in contact with their own private baths. Therefore, our perturbative calculations can help understand the anomalous heat transport in low dimensions, at least provide some benchmark for the numerical simulations and other analytical approximation schemes in the weakly nonlinear regime. Our predictions about the $\alpha$-FPUT, KG, and $\beta$-FPUT types of nonlinearities can be directly implemented and verified by e.g. engineering an array of Josephson junctions in the limit of large Josephson energy.

\section{Acknowledgement}

Work by JY and ANJ was supported by the U.S. Department of Energy (DOE), Office of Science, Basic Energy Sciences (BES) under Award No. DE-SC-0017890. JY would like to thank Professors A. Das and S. G. Rajeev for helpful discussions. JTH and BLH are thankful to Prof. Hong Zhao for his insight in nonlinear transport problems based on his extensive numerical work.

\section{Appendix A. Feynman-Vernon influence functional formalism}

With the help of the Feynman-Vernon influence functional formalism, the propagating function $\mathcal{J}\left(\chi_{f}, \chi_{f}^{\prime}, t_{f} ; \chi_{i}, \chi_{i}^{\prime}, t_{i}\right)$ can be formally expressed as

$$
J\left(\chi_{f}, \chi_{f}^{\prime}, t_{f} ; \chi_{i}, \chi_{i}^{\prime}, t_{i}\right)=\int_{\chi^{\left(t_{i}\right)=\chi_{i}}}^{\chi\left(t_{f}\right)=\chi_{f}} \mathcal{D} \chi \int_{\chi^{\prime}\left(t_{i}\right)=\chi_{i}^{\prime}}^{\chi^{\prime}\left(t_{f}\right)=\chi_{f}^{\prime}} \mathcal{D} \chi^{\prime} \exp \left\{i S_{\chi}[\chi]-i S_{\chi}\left[\chi^{\prime}\right]\right\} F\left[\chi, \chi^{\prime}\right],
$$

where the influence functional $F\left[\chi, \chi^{\prime}\right]$ in Eq. (A.1) is

$$
F\left[\chi, \chi^{\prime}\right]=\mathcal{F}[\boldsymbol{q}, \boldsymbol{r}]=\exp \left\{\int_{t_{i}}^{t_{f}} \int_{t_{i}}^{t_{f}} d s d s^{\prime}\left[i \boldsymbol{q}^{T}(s) \boldsymbol{G}_{R}\left(s, s^{\prime}\right) \boldsymbol{r}\left(s^{\prime}\right)-\frac{1}{2} \boldsymbol{q}^{T}(s) \boldsymbol{G}_{H}\left(s, s^{\prime}\right) \boldsymbol{q}\left(s^{\prime}\right)\right]\right\} .
$$

where we have introduced the center-of-mass coordinate $\boldsymbol{r}$ and relative coordinate $\boldsymbol{q}$

$$
\boldsymbol{q}(s)=\chi(s)-\chi^{\prime}(s), \quad \boldsymbol{r}(s)=\frac{1}{2}\left[\chi(s)+\chi^{\prime}(s)\right],
$$

and the $N \times N$ diagonal Green's function matrices

$$
\boldsymbol{G}_{R}\left(s, s^{\prime}\right)=\operatorname{diag}\left[G_{R}^{(n)}\left(s, s^{\prime}\right)\right], \quad \boldsymbol{G}_{H}\left(s, s^{\prime}\right)=\operatorname{diag}\left[G_{H}^{(n)}\left(s, s^{\prime}\right)\right] .
$$

The Green's functions $G_{R}^{(n)}\left(s, s^{\prime}\right)$ and $G_{H}^{(n)}\left(s, s^{\prime}\right)$ are respectively the dissipation kernel and the noise kernel of the private thermal bath at the temperature $\beta_{n}^{-1}$ associated with the $n^{\text {th }}$ oscillator

$$
\begin{aligned}
G_{R}^{(n)}\left(s, s^{\prime}\right) & =-\frac{e_{n}^{2}}{2 \pi} \theta\left(s-s^{\prime}\right) \partial_{s} \delta\left(s-s^{\prime}\right), & G_{H}^{(n)}\left(s, s^{\prime}\right)=e_{n}^{2} \int_{-\infty}^{\infty} d \omega \tilde{G}_{H}^{(n)}(\omega) e^{i \omega\left(s-s^{\prime}\right)}, \\
\tilde{G}_{H}^{(n)}(\omega) & =\omega \operatorname{coth}\left(\frac{\beta_{n} \omega}{2}\right), &
\end{aligned}
$$


and they are defined by

$$
\begin{aligned}
& G_{R}^{(n)}\left(s, s^{\prime}\right) \equiv i \theta\left(s-s^{\prime}\right)\left\langle\left[\hat{\phi}^{(i)}\left(z_{i}(s), s\right), \hat{\phi}^{(i)}\left(z_{i}\left(s^{\prime}\right), s^{\prime}\right)\right],\right\rangle \\
& G_{H}^{(n)}\left(s, s^{\prime}\right) \equiv\left\langle\left\{\hat{\phi}^{(i)}\left(z_{i}(s), s\right), \hat{\phi}^{(i)}\left(z_{i}\left(s^{\prime}\right), s^{\prime}\right)\right\}\right\rangle,
\end{aligned}
$$

where $\hat{\phi}_{n}\left(z_{n}(s), s\right)$ denotes the field operator in the Heisenberg picture and the expectation value is taken with respect to its initial thermal state.

Now it is useful to introduce the coarse-grained action

$$
\mathcal{S}_{\mathrm{CG}}[\boldsymbol{r}, \boldsymbol{q}]=\int_{t_{i}}^{t_{f}} d s\left\{\mathcal{S}_{\chi}\left[\boldsymbol{r}+\frac{\boldsymbol{q}}{2}\right]-\mathcal{S}_{\chi}\left[\boldsymbol{r}-\frac{\boldsymbol{q}}{2}\right]+\int_{t_{i}}^{t_{f}} d s^{\prime}\left[\boldsymbol{q}^{T}(s) \boldsymbol{G}_{R}\left(s, s^{\prime}\right) \boldsymbol{r}\left(s^{\prime}\right)+\frac{i}{2} \boldsymbol{q}^{T}(s) \boldsymbol{G}_{H}\left(s, s^{\prime}\right) \boldsymbol{q}\left(s^{\prime}\right)\right]\right\},
$$

and apply the Hubbard-Stratonovich transformation [17] to the term involving the noise kernel $\boldsymbol{G}_{H}\left(s, s^{\prime}\right)$ in Eq. (A.9), i.e.,

$$
\exp \left[-\frac{1}{2} \int_{t_{i}}^{t_{f}} d s \int_{t_{i}}^{t_{f}} d s^{\prime} \boldsymbol{q}^{T}(s) \boldsymbol{G}_{H}\left(s, s^{\prime}\right) \boldsymbol{q}\left(s^{\prime}\right)\right]=\int \mathcal{D} \boldsymbol{\xi} P[\boldsymbol{\xi}] \exp \left[i \int_{0}^{t_{f}} d s \boldsymbol{q}^{T}(s) \boldsymbol{\xi}(s)\right],
$$

where $P[\xi]$ is interpreted as the Gaussian probability distribution functional of the $c$-number stochastic noise $\boldsymbol{\xi}(s)$, whose first two moments are $\left\langle\xi_{k}(s)\right\rangle=0,\left\langle\xi_{k}(s) \xi_{l}\left(s^{\prime}\right)\right\rangle=G_{H}^{(k)}\left(s, s^{\prime}\right) \delta_{k l}$. This allows to introduce the stochastic propagating function

$$
\mathcal{J}_{\xi}\left(\boldsymbol{r}_{f}, \boldsymbol{q}_{f}, t_{f} ; \boldsymbol{r}_{i}, \boldsymbol{q}_{i}, t_{i}\right)=\int_{\boldsymbol{q}\left(t_{i}\right)=\boldsymbol{q}_{i}}^{\boldsymbol{q}\left(t_{f}\right)=\boldsymbol{q}_{f}} \mathcal{D} \boldsymbol{q} \int_{\boldsymbol{r}\left(t_{i}\right)=\boldsymbol{r}_{i}}^{\boldsymbol{r}\left(t_{f}\right)=\boldsymbol{r}_{f}} \mathcal{D} \boldsymbol{r} \exp \left\{i \mathcal{S}_{\xi}[\boldsymbol{r}, \boldsymbol{q}]\right\},
$$

where the stochastic effective action is defined as

$$
\mathcal{S}_{\xi}[\boldsymbol{r}, \boldsymbol{q}]=\int_{t_{i}}^{t_{f}} d s\left\{\mathcal{S}\left[\boldsymbol{r}+\frac{\boldsymbol{q}}{2}\right]-\mathcal{S}\left[\boldsymbol{r}-\frac{\boldsymbol{q}}{2}\right]+\int_{t_{i}}^{t_{f}} d s^{\prime} \boldsymbol{q}^{T}(s) \boldsymbol{G}_{R}\left(s, s^{\prime}\right) \boldsymbol{r}\left(s^{\prime}\right)+\boldsymbol{q}^{T}(s) \boldsymbol{\xi}(s)\right\} .
$$

\section{Appendix B. Functional method}

To evaluate the expectation value $O\left(t_{f}, \boldsymbol{\xi}\right]$ of the quantity $O$ for each realization of the stochastic noise, it is convenient to introduce the functional method. We first note that $\operatorname{Tr}\left\{\hat{\rho}\left(t_{f}\right)\right\}=\left\langle\left\langle\operatorname{Tr}\left\{\hat{\rho}_{\xi}\left(t_{f}\right)\right\}\right\rangle\right.$, and we can write

$$
\begin{aligned}
\operatorname{Tr}\left\{\hat{\rho}_{\xi}\left(t_{f}\right)\right\} & =\int d \chi_{i} d \chi_{i}^{\prime} \int d \chi_{f} J_{\xi}\left(\chi_{f}, \chi_{f}, t_{f} ; \chi_{i}, \chi_{i}^{\prime}\right) \rho\left(\chi_{i}, \chi_{i}^{\prime}\right) \\
& =\int d \boldsymbol{r}_{i} d \boldsymbol{q}_{i} \int d \boldsymbol{r}_{f} \mathcal{J}_{\xi}\left(\boldsymbol{r}_{f}, 0, t_{f} ; \boldsymbol{r}_{i}, \boldsymbol{q}_{i}, t_{i}\right) \varrho\left(\boldsymbol{r}_{i}, \boldsymbol{q}_{i}\right)
\end{aligned}
$$

where we have made the change of variables

$$
\chi_{f} \mapsto \boldsymbol{r}_{f}, \quad \chi_{i} \mapsto \boldsymbol{r}_{i}+\frac{\boldsymbol{q}_{i}}{2}, \quad \chi_{i}^{\prime} \mapsto \boldsymbol{r}_{i}-\frac{\boldsymbol{q}_{i}}{2},
$$

and introduced the functional variants of the stochastic propagating function $J_{\xi}\left(\chi_{f}, \chi_{f}, t_{f} ; \chi_{i}, \chi_{i}^{\prime}\right)$ and the density matrix $\rho\left(\chi_{i}, \chi_{i}^{\prime}\right)$ by

$$
J_{\xi}\left(\chi_{f}, \chi_{f}, t_{f} ; \chi_{i}, \chi_{i}^{\prime}\right)=\mathcal{J}_{\xi}\left(\boldsymbol{r}_{f}, 0, t_{f} ; \boldsymbol{r}_{i}, \boldsymbol{q}_{i}\right), \quad \rho\left(\chi_{i}, \chi_{i}^{\prime},\right)=\varrho\left(\boldsymbol{r}_{i}, \boldsymbol{q}_{i}\right),
$$


respectively. Thus, the expectation value $p_{n}^{2}\left(t_{f}, \xi\right]$ in (29) becomes

$$
\begin{aligned}
\operatorname{Tr}\left\{\hat{p}_{n}^{2} \hat{\rho}_{\xi}\left(t_{f}\right)\right\} & =\int d \boldsymbol{p}_{f} d \boldsymbol{\chi}_{f} d \boldsymbol{\chi}_{f}^{\prime}\left\langle\boldsymbol{\chi}_{f}^{\prime}\left|\hat{p}_{n}^{2}\right| \boldsymbol{p}_{f}\right\rangle\left\langle\boldsymbol{p}_{f} \mid \boldsymbol{\chi}_{f}\right\rangle\left\langle\boldsymbol{\chi}_{f}\left|\hat{\rho}_{\xi}\left(t_{f}\right)\right| \boldsymbol{\chi}_{f}^{\prime}\right\rangle \\
& =\frac{1}{(2 \pi)^{N}} \int d \boldsymbol{p}_{f} p_{f n}^{2} e^{i \boldsymbol{p}_{f} \cdot\left(\boldsymbol{\chi}_{f}^{\prime}-\boldsymbol{\chi}_{f}\right)} \int d \boldsymbol{\chi}_{f} d \boldsymbol{\chi}_{f}^{\prime}\left\langle\boldsymbol{\chi}_{f}\left|\hat{\rho}_{\xi}\left(t_{f}\right)\right| \boldsymbol{\chi}_{f}^{\prime}\right\rangle \\
& =\left.\frac{1}{(2 \pi)^{N} i^{2}} \frac{\partial^{2}}{\partial h_{n}^{2}} \int d \boldsymbol{p}_{f} e^{i \boldsymbol{p}_{f} \cdot\left(\boldsymbol{\chi}_{f}^{\prime}-\boldsymbol{\chi}_{f}\right)+i \boldsymbol{p}_{f} \cdot \boldsymbol{h}} \int d \boldsymbol{\chi}_{f} d \boldsymbol{\chi}_{f}^{\prime}\left\langle\boldsymbol{\chi}_{f}\left|\hat{\rho}\left(t_{f}\right)\right| \boldsymbol{\chi}_{f}^{\prime}\right\rangle\right|_{\boldsymbol{h}=0} \\
& =-\left.\frac{\partial^{2}}{\partial h_{n}^{2}} \int d \boldsymbol{\chi}_{f}\left\langle\boldsymbol{\chi}_{f}\left|\hat{\rho}\left(t_{f}\right)\right| \boldsymbol{\chi}_{f}-\boldsymbol{h}\right\rangle\right|_{\boldsymbol{h}=0} \\
& =-\left.\frac{\partial^{2}}{\partial h_{n}^{2}} \int d \boldsymbol{r}_{i} d \boldsymbol{q}_{i} \int d \boldsymbol{r}_{f} \mathcal{J}_{\xi}\left(\boldsymbol{r}_{f}, \boldsymbol{h}, t_{f} ; \boldsymbol{r}_{i}, \boldsymbol{q}_{i}\right) \varrho\left(\boldsymbol{r}_{i}, \boldsymbol{q}_{i}\right)\right|_{\boldsymbol{h}=0} .
\end{aligned}
$$

Similarly, we find the expectation value $p_{n}\left(t_{f}, \boldsymbol{\xi}\right]$ in (29) expressed by

$$
\operatorname{Tr}\left\{\hat{p}_{n} \hat{\rho}_{\xi}\left(t_{f}\right)\right\}=-\left.i \frac{\partial}{\partial h_{n}} \int d \boldsymbol{r}_{i} d \boldsymbol{q}_{i} \int d \boldsymbol{r}_{f} \mathcal{J}_{\xi}\left(\boldsymbol{r}_{f}, \boldsymbol{h}, t_{f} ; \boldsymbol{r}_{i}, \boldsymbol{q}_{i}\right) \varrho\left(\boldsymbol{r}_{i}, \boldsymbol{q}_{i}\right)\right|_{h=0} .
$$

as a derivative over a fictitious, external constant source $\boldsymbol{h}$.

It is straightforward to calculate $w_{v \rightarrow n}^{(\eta)}\left(t_{f}, \xi\right]$ in (30) by the same derivative approach. We need to calculate the following quantity

$$
\begin{aligned}
\operatorname{Tr}\left\{\hat{p}_{n}^{2}\left(\hat{\chi}_{n}-\hat{\chi}_{m}\right)^{\eta} \hat{\rho}_{\xi}\left(t_{f}\right)\right\} & =-\left.\frac{\partial^{2}}{\partial h_{n}^{2}} \int d \chi_{f}\left(\chi_{f n}-\chi_{f m}\right)^{\eta}\left\langle\chi_{f}\left|\hat{\rho}_{\xi}\left(t_{f}\right)\right| \chi_{f}-\boldsymbol{h}\right\rangle\right|_{\boldsymbol{h}=0} \\
& =-\left.\frac{\partial^{2}}{\partial h_{n}^{2}} \int d \boldsymbol{r}_{i} d \boldsymbol{q}_{i} \int d \boldsymbol{r}_{f}\left[r_{f n}+\frac{h_{n}}{2}-r_{f m}-\frac{h_{m}}{2}\right]^{\eta} \mathcal{J}_{\xi}\left(\boldsymbol{r}_{f}, \boldsymbol{h}, t_{f} ; \boldsymbol{r}_{i}, \boldsymbol{q}_{i}\right) \varrho\left(\boldsymbol{r}_{i}, \boldsymbol{q}_{i}\right)\right|_{\boldsymbol{h}=0},
\end{aligned}
$$

and the quantity

$$
\begin{aligned}
\operatorname{Tr}\left\{\left(\hat{\chi}_{n}-\hat{\chi}_{m}\right)^{\eta} \hat{p}_{n}^{2} \hat{\rho}_{\xi}\left(t_{f}\right)\right\} & =-\left.\frac{\partial^{2}}{\partial h_{n}^{2}} \int d \chi_{f}\left(\chi_{n}-\chi_{m}\right)^{\eta}\left\langle\chi_{f}+\boldsymbol{h}\left|\hat{\rho}_{\xi}\left(t_{f}\right)\right| \chi_{f}\right\rangle\right|_{\boldsymbol{h}=0} \\
& =-\left.\frac{\partial^{2}}{\partial h_{n}^{2}} \int d \boldsymbol{r}_{i} d \boldsymbol{q}_{i} \int d \boldsymbol{r}_{f}\left[r_{f n}-\frac{h_{n}}{2}-r_{f m}+\frac{h_{m}}{2}\right]^{\eta} \mathcal{J}_{\xi}\left(\boldsymbol{r}_{f}, \boldsymbol{h}, t_{f} ; \boldsymbol{r}_{i}, \boldsymbol{q}_{i}\right) \varrho\left(\boldsymbol{r}_{i}, \boldsymbol{q}_{i}\right)\right|_{\boldsymbol{h}=0} .
\end{aligned}
$$

Note the different signs in the right hand sides in Eq. (B.6, B.7) due to the differential positions of $\hat{p}_{n}$ on the left hand sides. Since the derivatives in Eqs. (B.6), (B.7) do not involve $h_{m}(m \neq n)$, so we can safely set $h_{m}=0$ in these equation. Furthermore, the terms proportional to the third power of $h_{n}$ and higher can be discarded since only the second-order derivatives of $h_{n}$ are involved. With these observations, we immediately find

$$
\begin{aligned}
\operatorname{Tr}\left\{\left[\hat{p}_{n}^{2},\left(\hat{\chi}_{n}-\hat{\chi}_{m}\right)^{2}\right] \hat{\rho}_{\xi}\left(t_{f}\right)\right\} & =-\left.2 \frac{\partial^{2}}{\partial h_{n}^{2}} \int d \boldsymbol{r}_{i} d \boldsymbol{q}_{i} \int d \boldsymbol{r}_{f} h_{n}\left[r_{f n}-r_{f m}\right] \mathcal{J}_{\bar{\xi}}\left(\boldsymbol{r}_{f}, \boldsymbol{h}, t_{f} ; \boldsymbol{r}_{i}, \boldsymbol{q}_{i}\right) \varrho\left(\boldsymbol{r}_{i}, \boldsymbol{q}_{i}\right)\right|_{\boldsymbol{h}=0} \\
& =-\left.2 \frac{\partial^{2}}{\partial h_{n}^{2}}\left\{h_{n}\left[\left\langle r_{f n}\right\rangle_{\boldsymbol{h}}-\left\langle r_{f m}\right\rangle_{\boldsymbol{h}}\right]\right\}\right|_{\boldsymbol{h}=0} \\
& =-\left.4 \frac{\partial}{\partial h_{n}}\left\langle r_{f n}-r_{f m}\right\rangle_{\boldsymbol{h}}\right|_{\boldsymbol{h}=0},
\end{aligned}
$$

where the expectation value $\langle\bullet\rangle$ is defined by

$$
\langle\bullet\rangle \equiv \operatorname{Tr}\left\{\bullet \hat{\rho}\left(t_{f}\right)\right\}=\int d \boldsymbol{r}_{i} d \boldsymbol{q}_{i} \int d \boldsymbol{r}_{f}(\bullet) \mathcal{J}_{\xi}\left(\boldsymbol{r}_{f}, \boldsymbol{h}, t_{f} ; \boldsymbol{r}_{i}, \boldsymbol{q}_{i}\right) \varrho\left(\boldsymbol{r}_{i}, \boldsymbol{q}_{i}\right),
$$

and the subscript $\boldsymbol{h}$ is a reminder that this expectation value is still a function of $\boldsymbol{h}$. Similarly, We can find

$$
\begin{aligned}
\operatorname{Tr}\left\{\left[\hat{p}_{n}^{2},\left(\hat{\chi}_{n}-\hat{\chi}_{m}\right)^{3}\right] \hat{\rho}_{\xi}\left(t_{f}\right)\right\} & =-\left.3 \frac{\partial^{2}}{\partial h_{n}^{2}} \int d \boldsymbol{r}_{i} d \boldsymbol{q}_{i} \int d \boldsymbol{r}_{f} h_{n}\left[r_{f n}-r_{f m}\right]^{2} \mathcal{J}_{\xi}\left(\boldsymbol{r}_{f}, \boldsymbol{h}, t_{f} ; \boldsymbol{r}_{i}, \boldsymbol{q}_{i}\right) \varrho\left(\boldsymbol{r}_{i}, \boldsymbol{q}_{i}\right)\right|_{\boldsymbol{h}=0} \\
& =-\left.6 \frac{\partial}{\partial h_{n}}\left\langle\left(r_{f n}-r_{f m}\right)^{2}\right\rangle_{\boldsymbol{h}}\right|_{\boldsymbol{h}=0},
\end{aligned}
$$


and

$$
\operatorname{Tr}\left\{\left[\hat{p}_{n}^{2},\left(\hat{\chi}_{n}-\hat{\chi}_{m}\right)^{4}\right] \hat{\rho}_{\xi}\left(t_{f}\right)\right\}=-\left.8 \frac{\partial}{\partial h_{n}}\left\langle\left(r_{f n}-r_{f m}\right)^{3}\right\rangle_{\boldsymbol{h}}\right|_{\boldsymbol{h}=0} .
$$

Note that in Eqs. (B.4-B.11), oscillator $n$ and $m$ are not necessarily ajacent to each other and interactions beyond the nearest neigbhor interactions are also included. If we take $m=v$ with $v=n \pm 1$ we arrive at a simple, but general expression for $w_{v \rightarrow n}^{(\eta)}\left(t_{f}, \xi\right]$

$$
w_{v \rightarrow n}^{(\eta)}\left(t_{f}, \boldsymbol{\xi}\right]=\left.i \frac{\partial}{\partial h_{n}}\left\langle\left(r_{f n}-r_{f v}\right)^{\eta-1}\right\rangle_{\boldsymbol{h}}\right|_{\boldsymbol{h}=0},
$$

In calculating Eqs. (B.4-B.11), we note that it turns out convenient to introduce the generating functional

$$
\mathcal{Z}_{\xi}\left[\boldsymbol{j}, \boldsymbol{j}^{\prime}, \boldsymbol{h}\right)=\int d \boldsymbol{r}_{i} d \boldsymbol{q}_{i} \int d \boldsymbol{r}_{f} \int_{\boldsymbol{r}(0)=\boldsymbol{r}_{i}}^{\boldsymbol{r}\left(t_{f}\right)=\boldsymbol{r}_{f}} \mathcal{D} \boldsymbol{r} \int_{\boldsymbol{q}(0)=\boldsymbol{q}_{i}}^{\boldsymbol{q}\left(t_{f}\right)=\boldsymbol{h}} \mathcal{D} \boldsymbol{q} e^{i \mathcal{S}_{\xi}\left[\boldsymbol{r}, \boldsymbol{q} ; \boldsymbol{j}, j^{\prime}\right]} \varrho\left(\boldsymbol{r}_{i}, \boldsymbol{q}_{i}\right),
$$

with a new action $\mathcal{S}_{\xi}\left[\boldsymbol{r}, \boldsymbol{q} ; \boldsymbol{j}, \boldsymbol{j}^{\prime}\right]$ defined by

$$
\mathcal{S}_{\xi}\left[\boldsymbol{r}, \boldsymbol{q} ; \boldsymbol{j}, \boldsymbol{j}^{\prime}\right]=\mathcal{S}_{\xi}[\boldsymbol{r}, \boldsymbol{q}]+\int_{0}^{t_{f}} d s\left\{\boldsymbol{j}(s) \cdot \boldsymbol{r}(s)+\boldsymbol{j}^{\prime}(s) \cdot \boldsymbol{q}(s)\right\}
$$

Apparently it is the stochastic effective action $\mathcal{S}_{\xi}[\boldsymbol{r}, \boldsymbol{q}]$ in (A.12) attached with external sources $\boldsymbol{j}$ and $\boldsymbol{j}^{\prime}$. With the help of this generating functional, the results in Eqs. (34) and (35) then take very succinct forms.

To find the energy exchange between the oscillators, we need to evaluate $w_{v \rightarrow n}^{(\eta)}\left(t_{f}, \xi\right]$ in (30). Generically it is equivalent to the evaluations of the multi-moment of $\chi$. We can do it also by the functional method. For example, the second moment $\operatorname{Tr}\left\{\hat{\chi}_{m} \hat{\chi}_{n} \hat{\rho}_{\xi}\left(t_{f}\right)\right\}$ can be found by

$$
\operatorname{Tr}\left\{\hat{\chi}_{n} \hat{\chi}_{m} \hat{\rho}_{\xi}\left(t_{f}\right)\right\}=\int d \chi_{f} \chi_{f n} \chi_{f m} \rho_{\xi}\left(\chi_{f}, \chi_{f}, t_{f}\right)=\left\langle r_{n}\left(t_{f}\right) r_{m}\left(t_{f}\right)\right\rangle_{\boldsymbol{h}=0},
$$

and, similarly, three and four-point moments are given by

$$
\begin{aligned}
\operatorname{Tr}\left\{\hat{\chi}_{n} \hat{\chi}_{m} \hat{\chi}_{k} \hat{\rho}_{\xi}\left(t_{f}\right)\right\} & =\left\langle r_{n}\left(t_{f}\right) r_{m}\left(t_{f}\right) r_{k}\left(t_{f}\right)\right\rangle_{\boldsymbol{h}=0}, \\
\operatorname{Tr}\left\{\hat{\chi}_{n} \hat{\chi}_{m} \hat{\chi}_{k} \hat{\chi}_{l} \hat{\rho}_{\xi}\left(t_{f}\right)\right\} & =\left\langle r_{n}\left(t_{f}\right) r_{m}\left(t_{f}\right) r_{k}\left(t_{f}\right) r_{l}\left(t_{f}\right)\right\rangle_{\boldsymbol{h}=0},
\end{aligned}
$$

with

$$
\begin{gathered}
\left\langle r_{n}\left(t_{f}\right) r_{m}\left(t_{f}\right)\right\rangle_{\boldsymbol{h}=0}=-\left.\frac{\delta^{2} \mathcal{Z}_{\xi}\left[\boldsymbol{j}, \boldsymbol{j}^{\prime}, \boldsymbol{h}\right)}{\delta j_{n}\left(t_{f}\right) \delta j_{m}\left(t_{f}\right)}\right|_{\boldsymbol{j}=\boldsymbol{j}^{\prime}=\boldsymbol{h}=0}, \\
\left\langle r_{n}\left(t_{f}\right) r_{m}\left(t_{f}\right) r_{k}\left(t_{f}\right) r_{l}\left(t_{f}\right)\right\rangle_{\boldsymbol{h}=0}=+\left.\frac{\delta^{4} \mathcal{Z}_{\xi}\left[\boldsymbol{j}, \boldsymbol{j}^{\prime}, \boldsymbol{h}\right)}{\delta j_{n}\left(t_{f}\right) \delta j_{m}\left(t_{f}\right) \delta j_{k}\left(t_{f}\right) \delta j_{l}\left(t_{f}\right)}\right|_{\boldsymbol{j}=j^{\prime}=\boldsymbol{h}=0} .
\end{gathered}
$$

Here we see that the generating functional defined in (37) can be used to efficiently to evaluate the quantities such as $\operatorname{Tr}\left\{\hat{p}_{n_{1}} \cdots \hat{p}_{n_{a}} \hat{\chi}_{m_{1}} \cdots \hat{\chi}_{m_{b}} \hat{\rho}_{\xi}\left(t_{f}\right)\right\}$ or $\operatorname{Tr}\left\{\hat{\chi}_{m_{1}} \cdots \hat{\chi}_{m_{b}} \hat{\rho}_{\xi}\left(t_{f}\right) \hat{p}_{n_{1}} \cdots \hat{p}_{n_{a}}\right\}$ for any two arbitrary nonnegative integers $a$ and $b$.

\section{Appendix C. Explicit evaluation of the noninteracting stochastic propagating function}

Here we will derive the explicit form of the stochastic propagating function for the linearly coupled oscillators. This will be the zeroth-order term for the more general configuration considered in the paper, and will serve as the basis for the perturbative calculations of the higher-order corrections in various nonlinear coupling discussed earlier.

Taking variations of $\mathcal{S}_{\xi}^{(0)}$ in (43) with respect to $\boldsymbol{q}$ and $\boldsymbol{r}$ respectively, we obtains a sets of differential equations for the classical trajectories $\overline{\boldsymbol{q}}$ and $\overline{\boldsymbol{r}}$,

$$
\begin{aligned}
& \ddot{\overline{\boldsymbol{r}}}(s)-\int_{0}^{t_{f}} d s^{\prime} \boldsymbol{G}_{R}\left(s, s^{\prime}\right) \cdot \overline{\boldsymbol{r}}\left(s^{\prime}\right)+\boldsymbol{\Omega}^{2} \overline{\boldsymbol{r}}(s)=\boldsymbol{j}^{\prime}(s)+\boldsymbol{\xi}(s), \\
& \ddot{\overline{\boldsymbol{q}}}(s)-\int_{0}^{t_{f}} d s^{\prime} \boldsymbol{G}_{R}\left(s^{\prime}, s\right) \cdot \overline{\boldsymbol{q}}\left(s^{\prime}\right)+\boldsymbol{\Omega}^{2} \overline{\boldsymbol{q}}(s)=\boldsymbol{j}(s),
\end{aligned}
$$


with boundary conditions $\overline{\boldsymbol{r}}\left(t_{f}\right)=\boldsymbol{r}_{f}, \overline{\boldsymbol{r}}(0)=\boldsymbol{r}_{i}, \overline{\boldsymbol{q}}\left(t_{f}\right)=\boldsymbol{q}_{f}, \overline{\boldsymbol{q}}(0)=\boldsymbol{q}_{i}$, and $t_{i}=0$.

In fact the integrals in Eqs. (C.1) and (C.2) can be computed explicitly using Eq. (A.5). We find

$$
\begin{aligned}
& \int_{0}^{t_{f}} d s^{\prime} \boldsymbol{G}_{R}\left(s, s^{\prime}\right) \cdot \overline{\boldsymbol{r}}\left(s^{\prime}\right)=-2 \gamma[\delta(0) \overline{\boldsymbol{r}}(s)+\dot{\overline{\boldsymbol{r}}}(s)], \\
& \int_{0}^{t_{f}} d s^{\prime} \boldsymbol{G}_{R}\left(s^{\prime}, s\right) \cdot \overline{\boldsymbol{q}}\left(s^{\prime}\right)=2 \gamma[\delta(0) \overline{\boldsymbol{q}}(s)+\dot{\overline{\boldsymbol{q}}}(s)],
\end{aligned}
$$

with $\gamma=e^{2} / 8 \pi$. Eqs. (C.1) and (C.2) then become

$$
\begin{aligned}
\ddot{\boldsymbol{r}}(s)+2 \gamma \dot{\overline{\boldsymbol{r}}}(s)+\boldsymbol{\Omega}_{R}^{2} \cdot \overline{\boldsymbol{r}}(s) & =\boldsymbol{j}^{\prime}(s)+\boldsymbol{\xi}(s), \\
\ddot{\boldsymbol{q}}(s)-2 \gamma \dot{\overline{\boldsymbol{q}}}(s)+\mathbf{\Omega}_{R}^{2} \cdot \overline{\boldsymbol{q}}(s) & =\boldsymbol{j}(s) .
\end{aligned}
$$

where the divergent expression $\delta(0)$ is absorbed by $\omega_{0}$ in $\boldsymbol{\Omega}$ to give the renormalized value $\omega_{R}$, and thus the renormalized frequency matrix $\boldsymbol{\Omega}_{R}^{2}$ is defined as

$$
\boldsymbol{\Omega}_{R}^{2} \equiv\left[\begin{array}{cc}
\omega_{R}^{2}+\lambda_{2} & -\lambda_{2} \\
-\lambda_{2} & \omega_{R}^{2}+\lambda_{2}
\end{array}\right] .
$$

Following the procedures outlined in [17, 27], we find the classical stochastic action $\bar{S}_{\xi}^{(0)}\left[\overline{\boldsymbol{q}}, \overline{\boldsymbol{r}}, \boldsymbol{j}, \boldsymbol{j}^{\prime}\right]$ given by

$$
\begin{aligned}
\overline{\mathcal{S}}_{\xi}^{(0)}\left[\boldsymbol{j}, \boldsymbol{j}^{\prime} ; \boldsymbol{r}_{f}, \boldsymbol{q}_{f}, t_{f} ; \boldsymbol{r}_{i}, \boldsymbol{q}_{i}\right)= & \boldsymbol{q}_{f}^{T} \cdot\left\{\dot{\boldsymbol{\alpha}}\left(t_{f}\right) \cdot \boldsymbol{r}_{i}+\dot{\boldsymbol{\beta}}\left(t_{f}\right) \cdot \boldsymbol{r}_{f}\right\}-\boldsymbol{q}_{i}^{T} \cdot\left\{\dot{\boldsymbol{\alpha}}(0) \cdot \boldsymbol{r}_{i}+\dot{\boldsymbol{\beta}}(0) \cdot \boldsymbol{r}_{f}\right\} \\
& +\boldsymbol{q}_{f}^{T} \cdot\left\{\dot{\boldsymbol{F}}\left(t_{f}, \boldsymbol{j}^{\prime}\right]+\dot{\boldsymbol{F}}\left(t_{f}, \boldsymbol{\xi}\right]\right\}-\boldsymbol{q}_{i}^{T} \cdot\left\{\dot{\boldsymbol{F}}\left(0, \boldsymbol{j}^{\prime}\right]+\dot{\boldsymbol{F}}(0, \boldsymbol{\xi}]\right\} \\
& +\int_{0}^{t_{f}} d s \dot{\boldsymbol{j}}^{T}(s) \cdot \boldsymbol{\alpha}(s) \cdot \boldsymbol{r}_{i}+\int_{0}^{t_{f}} d s \dot{\boldsymbol{j}}^{T}(s) \cdot \boldsymbol{\beta}(s) \cdot \boldsymbol{r}_{f}+\int_{0}^{t_{f}} d s \boldsymbol{j}^{T}(s) \cdot\left\{\boldsymbol{F}\left(s, \boldsymbol{j}^{\prime}\right]+\boldsymbol{F}(s, \boldsymbol{\xi}]\right\} .
\end{aligned}
$$

Here we have introduced a couple of shorthand notations

$$
\begin{array}{rrr}
\boldsymbol{\alpha}(t) & =\boldsymbol{D}_{1}(t)-\boldsymbol{D}_{2}(t) \cdot \boldsymbol{D}_{2}^{-1}\left(t_{f}\right) \cdot \boldsymbol{D}_{1}\left(t_{f}\right), & \boldsymbol{\beta}(t)=\boldsymbol{D}_{2}(t) \cdot \boldsymbol{D}_{2}^{-1}\left(t_{f}\right), \\
\boldsymbol{F}(t, \boldsymbol{f}] & =\int_{0}^{t} d s \boldsymbol{D}_{2}(t-s) \cdot \boldsymbol{f}(s)-\boldsymbol{D}_{2}(t) \cdot \boldsymbol{D}_{2}^{-1}\left(t_{f}\right) \cdot \int_{0}^{t_{f}} d s \boldsymbol{D}_{2}\left(t_{f}-s\right) \cdot \boldsymbol{f}(s),
\end{array}
$$

among which $\boldsymbol{D}_{1}(t)$ and $\boldsymbol{D}_{2}(t)$ two fundamental solutions to (C.5), satisfying the initial conditions $\boldsymbol{D}_{1}(0)=\mathbb{I}, \dot{\boldsymbol{D}}_{1}(0)=$ $0, \boldsymbol{D}_{2}(0)=0$ and $\dot{\boldsymbol{D}}_{2}(0)=\mathbb{I}$.

Using the Laplace transform, we can find the explicit forms of $\boldsymbol{D}_{1}$ and $\boldsymbol{D}_{2}$

$$
\begin{aligned}
& \boldsymbol{D}_{1}(\mathfrak{s})=(\mathfrak{s}+2 \gamma)\left[\mathfrak{s}^{2}+2 \gamma_{\mathfrak{s}}+\boldsymbol{\Omega}_{R}^{2}\right]^{-1}=\frac{\boldsymbol{W}_{1}(\mathfrak{s})}{\prod_{k=1}^{4}\left(\mathfrak{s}-\mathfrak{s}_{k}\right)} \\
& \boldsymbol{D}_{2}(\mathfrak{s})=\left[\mathfrak{s}^{2}+2 \gamma_{\mathfrak{s}}+\boldsymbol{\Omega}_{R}^{2}\right]^{-1}=\frac{\boldsymbol{W}_{2}(\mathfrak{s})}{\prod_{k=1}^{4}\left(\mathfrak{s}-\mathfrak{s}_{k}\right)}
\end{aligned}
$$

where $\boldsymbol{W}_{1}(\mathfrak{s})=(\mathfrak{s}+2 \gamma) \boldsymbol{W}_{2}(\mathfrak{s})$,

$$
\boldsymbol{W}_{2}(\mathfrak{s})=\left[\begin{array}{cc}
\mathfrak{s}^{2}+2 \gamma_{\mathfrak{s}}+\omega_{0}^{2}+\lambda_{2}, & \lambda_{2} \\
\lambda_{2}, & \mathfrak{s}^{2}+2 \gamma_{\mathfrak{s}}+\omega_{0}^{2}+\lambda_{2}
\end{array}\right],
$$

with $\mathfrak{s}_{1,2}=-\gamma \pm \sqrt{\gamma^{2}-\omega_{0}^{2}}$ and $\mathfrak{s}_{3,4}=-\gamma \pm \sqrt{\gamma^{2}-\left(\omega_{0}^{2}+2 \lambda_{2}\right)}$. Since $\operatorname{Re}_{\mathfrak{s}_{k}}<0$ for all $k=1, \cdots, 4$, all the matrix elements of $\boldsymbol{D}_{i}(t)$ exponentially decay on time scale much large than $\gamma_{0}^{-1}$, where

$$
\gamma_{0} \equiv \min _{k}\left|\operatorname{Re} \mathfrak{s}_{k}\right|,
$$

i.e., $\boldsymbol{D}_{1,2}(t) \sim e^{-\gamma_{0} t}$, which leads to

$$
\lim _{t \rightarrow \infty} \frac{d^{n}}{d t^{n}} \boldsymbol{D}_{i}(t)=0
$$


where $n=0,1,2 \cdots$. This is an extremely useful property in the context of the late-time dynamics of the oscillators. Essentially, it predicts that the zeroth-order dynamics of the oscillators will relax to a steady state, independent of the initial conditions.

Since from (A.11), the integral gives

$$
\begin{aligned}
& \int d \boldsymbol{r}_{f} \exp \left\{i\left[\boldsymbol{q}_{f}^{T} \cdot \dot{\boldsymbol{\beta}}\left(t_{f}\right)-\boldsymbol{q}_{i}^{T} \cdot \dot{\boldsymbol{\beta}}(0)+\int_{0}^{t_{f}} d s \dot{j}^{T}(s) \cdot \boldsymbol{\beta}(s)\right] \cdot \boldsymbol{r}_{f}\right\} \\
= & \mathcal{N}^{-1} \delta^{(N)}\left(\boldsymbol{q}_{i}^{T}-\boldsymbol{q}_{f}^{T} \cdot \dot{\boldsymbol{D}}_{2}\left(t_{f}\right)-\int_{0}^{t_{f}} d s \boldsymbol{j}^{T}(s) \cdot \boldsymbol{D}_{2}(s)\right),
\end{aligned}
$$

with the normalization $\mathcal{N}^{-1}=(2 \pi)^{N} \operatorname{det} \boldsymbol{D}_{2}\left(t_{f}\right)$, we arrive at

$$
\begin{aligned}
& \int d \boldsymbol{r}_{f} \mathcal{J}_{0 \xi}\left[\boldsymbol{j}, \boldsymbol{j}^{\prime} ; \boldsymbol{r}_{f}, \boldsymbol{h}, t_{f} ; \boldsymbol{r}_{i}, \boldsymbol{q}_{i}\right) \\
= & \delta^{(N)}\left(\boldsymbol{q}_{i}^{T}-\boldsymbol{q}_{f}^{T} \cdot \dot{\boldsymbol{D}}_{2}\left(t_{f}\right)-\int_{0}^{t_{f}} d s \dot{j}^{T}(s) \cdot \boldsymbol{D}_{2}(s)\right) \times \exp \left\{i \overline{\mathcal{S}}_{\xi}^{(0)}\left[\boldsymbol{j}, \boldsymbol{j}^{\prime} ; \mathbf{0}, \boldsymbol{h}, t_{f} ; \boldsymbol{r}_{i}, \boldsymbol{q}_{i}\right)\right\},
\end{aligned}
$$

from Eq. (C.16). Thus, the stochastic generating function $\mathcal{Z}_{\xi}^{(0)}$ at late times reduces to

$$
\mathcal{Z}_{\xi}^{(0)}\left[\boldsymbol{j}, \boldsymbol{j}^{\prime}, \boldsymbol{h}\right)=\int d \boldsymbol{r}_{i} \exp \left\{i \overline{\mathcal{S}}_{\xi}^{(0)}\left[\boldsymbol{j}, \boldsymbol{j}^{\prime} ; \mathbf{0}, \boldsymbol{h}, t_{f} ; \boldsymbol{r}_{i}, \mathbf{0}\right)\right\} \varrho\left(\boldsymbol{r}_{i}, \int_{0}^{t_{f}} d s \boldsymbol{D}_{2}(s) \boldsymbol{j}(s)\right),
$$

where we have removed terms that will vanish in the limit $t_{f} \rightarrow 0$. The stochastic action in this limit will take a simpler form

$$
\begin{gathered}
\overline{\mathcal{S}}_{\xi}^{(0)}\left[\boldsymbol{j}, \boldsymbol{j}^{\prime} ; \mathbf{0}, \boldsymbol{h}, t_{f} ; \boldsymbol{r}_{i}, \mathbf{0}\right)=\int_{0}^{t_{f}} d s \boldsymbol{j}^{T}(s) \cdot \boldsymbol{D}_{1}(s) \cdot \boldsymbol{r}_{i}+\boldsymbol{h}^{T} \cdot \int_{0}^{t_{f}} d s \dot{\boldsymbol{D}}_{2}\left(t_{f}-s\right) \cdot \boldsymbol{\xi}(s)+\boldsymbol{h}^{T} \cdot \int_{0}^{t_{f}} d s \dot{\boldsymbol{D}}_{2}\left(t_{f}-s\right) \cdot \boldsymbol{j}^{\prime}(s) \\
+\int_{0}^{t_{f}} d s \int_{0}^{s} d s^{\prime} \boldsymbol{j}^{T}(s) \cdot \boldsymbol{D}_{2}\left(s-s^{\prime}\right) \cdot\left[\boldsymbol{j}^{\prime}\left(s^{\prime}\right)+\boldsymbol{\xi}\left(s^{\prime}\right)\right]
\end{gathered}
$$

\section{Appendix D. Theorems on the functional derivatives}

In this section, we have gathered a few handy theorems about the functional derivatives used in the calculations of the first-order corrections to the energy current in the anharmonic chain.

Theorem 1. For $v+\kappa<\mu$

$$
\left.\frac{\partial^{\kappa} \delta^{\nu+\mu} \mathcal{Z}_{\xi}^{(0)}\left[\boldsymbol{j}, \boldsymbol{j}^{\prime}, \boldsymbol{h}\right)}{\partial h_{k_{1}} \partial h_{k_{2}} \cdots \partial h_{k_{k}} \delta j_{n_{1}}\left(s_{1}\right) \delta j_{n_{2}}\left(s_{2}\right) \cdots \delta j_{n_{\nu}}\left(s_{\nu}\right) \delta j_{m_{1}}^{\prime}\left(s_{1}^{\prime}\right) \delta j_{m_{2}}^{\prime}\left(s_{2}^{\prime}\right) \cdots \delta j_{m_{\mu}}^{\prime}\left(s_{\mu}^{\prime}\right)}\right|_{\boldsymbol{j}=j^{\prime}=\boldsymbol{h}=0}=0,
$$

where $\kappa, v$ and $\mu$ are non-negative integers.

Proof. The Taylor's expanding of the term $\exp \left\{i \bar{\Xi}^{(0)}\left[\boldsymbol{j}, \boldsymbol{j}^{\prime}, \boldsymbol{h}\right)\right\}$ gives defined Eq. (48),

$$
\exp \left\{i \bar{\Xi}^{(0)}\left[\boldsymbol{j}, \boldsymbol{j}^{\prime}, \boldsymbol{h}\right)\right\}=\sum_{n=0}^{\infty} \frac{i^{n}}{n !}\left[\int_{0}^{t_{f}} d s \int_{0}^{t_{f}} d s^{\prime} \boldsymbol{j}^{T}(s) \cdot \mathfrak{D}\left(s-s^{\prime}\right) \cdot \boldsymbol{j}^{\prime}\left(s^{\prime}\right)+\boldsymbol{h}^{T} \cdot \int_{0}^{t_{f}} d s \dot{\boldsymbol{D}}_{2}\left(t_{f}-s\right) \cdot \boldsymbol{j}^{\prime}(s)\right]^{n} .
$$

By counting the powers of $\boldsymbol{j}^{\prime}$, we see that after we take the functional derivatives $\delta^{\mu} / \delta j_{m_{1}}^{\prime}\left(s_{1}^{\prime}\right) \delta j_{m_{2}}^{\prime}\left(s_{2}^{\prime}\right) \cdots \delta j_{m_{\mu}}^{\prime}\left(s_{\mu}^{\prime}\right)$ and set $\boldsymbol{j}^{\prime}=0$, the only nonvanishing contributions in the Taylor series is

$$
\frac{i^{\mu}}{\mu !}\left[\int_{0}^{t_{f}} d s \int_{0}^{t_{f}} d s^{\prime} \boldsymbol{j}^{T}(s) \cdot \mathfrak{D}\left(s-s^{\prime}\right) \cdot \boldsymbol{j}^{\prime}\left(s^{\prime}\right)+\boldsymbol{h}^{T} \cdot \int_{0}^{t_{f}} d s \dot{\boldsymbol{D}}_{2}\left(t_{f}-s\right) \cdot \boldsymbol{j}^{\prime}(s)\right]^{\mu} .
$$

Then we immediate see that the total powers of $\boldsymbol{j}$ and $\boldsymbol{h}$ in the expansion of Eq. (D.3) must be $\mu$, too. If we take the subsequent functional derivatives $\partial^{\kappa} \delta^{v} / \delta j_{n_{1}}\left(s_{1}\right) \delta j_{n_{2}}\left(s_{2}\right) \cdots \delta j_{n_{v}}\left(s_{v}\right) \partial h_{k_{1}} \partial h_{k_{2}} \cdots \partial h_{k_{k}}$ of (D.3), then we are left with term that are at least $(\mu-v-\kappa)^{\text {th }}$ power of $\boldsymbol{j}$ or $\boldsymbol{h}$. Setting $\boldsymbol{j}=\boldsymbol{j}^{\prime}=\boldsymbol{h}=0$ gives vanishing results. 
Theorem 2. For the case of $v+\kappa=\mu$,

$$
\begin{aligned}
& \left.\frac{\partial^{\kappa} \delta^{v+\mu} \mathcal{Z}_{\boldsymbol{\xi}}^{(0)}\left[\boldsymbol{j}, \boldsymbol{j}^{\prime}, \boldsymbol{h}\right)}{\partial h_{k_{1}} \partial h_{k_{2}} \cdots \partial h_{k_{k}} \delta j_{n_{1}}\left(s_{1}\right) \delta j_{n_{2}}\left(s_{2}\right) \cdots \delta j_{n_{v}}\left(s_{v}\right) \delta j_{m_{1}}^{\prime}\left(s_{1}^{\prime}\right) \delta j_{m_{2}}^{\prime}\left(s_{2}^{\prime}\right) \cdots \delta j_{m_{\mu}}^{\prime}\left(s_{\mu}^{\prime}\right)}\right|_{j=j^{\prime}=\boldsymbol{h}=0} \\
= & \frac{i^{\mu}}{\mu !} C_{\kappa}^{\mu} \frac{\delta^{\nu+\mu}\left[\int_{0}^{t_{f}} d s \int_{0}^{t_{f}} d s^{\prime} \boldsymbol{j}^{T}(s) \cdot \mathfrak{D}\left(s-s^{\prime}\right) \cdot \boldsymbol{j}^{\prime}\left(s^{\prime}\right)\right]^{\mu-\kappa}\left[\boldsymbol{h}^{T} \cdot \int_{0}^{t_{f}} d s \dot{\boldsymbol{D}}_{2}\left(t_{f}-s\right) \cdot \boldsymbol{j}^{\prime}(s)\right]_{j=j^{\prime}=\boldsymbol{h}=0}^{K}}{\delta j_{n_{1}}\left(s_{1}\right) \delta j_{n_{2}}\left(s_{2}\right) \cdots \delta j_{n_{v}}\left(s_{v}\right) \delta j_{m_{1}}^{\prime}\left(s_{1}^{\prime}\right) \delta j_{m_{2}}^{\prime}\left(s_{2}^{\prime}\right) \cdots \delta j_{m_{\mu}}^{\prime}\left(s_{\mu}^{\prime}\right)}
\end{aligned}
$$

which may be further evaluated analytically using the Wick's theorem.

Proof. The proof is similar to the proof for Theorem 1. After we take the functional derivatives $\delta^{\mu} / \delta j_{m_{1}}^{\prime}\left(s_{1}^{\prime}\right) \cdots \delta j_{m_{\mu}}^{\prime}\left(s_{\mu}^{\prime}\right)$ of the Taylor's expansion of Eq. (D.2) and set $\boldsymbol{j}^{\prime}=0$, we find the surviving terms in the expansion are

$$
\frac{i^{\mu}}{\mu !}\left[\int_{0}^{t_{f}} d s \int_{0}^{t_{f}} d s^{\prime} \boldsymbol{j}^{T}(s) \cdot \mathfrak{D}\left(s-s^{\prime}\right) \cdot \boldsymbol{j}^{\prime}\left(s^{\prime}\right)+\boldsymbol{h}^{T} \cdot \int_{0}^{t_{f}} d s \dot{\boldsymbol{D}}_{2}\left(t_{f}-s\right) \cdot \boldsymbol{j}^{\prime}(s)\right]^{\mu} .
$$

Applying the additonal derivatives $\partial^{\kappa} \delta^{\nu} / \partial h_{k_{1}} \partial h_{k_{2}} \cdots \partial h_{k_{\kappa}} \delta j_{n_{1}}\left(s_{1}\right) \delta j_{n_{2}}\left(s_{2}\right) \cdots \delta j_{n_{\mu}}\left(s_{\nu}\right)$ on (D.5) gives

$$
\frac{i^{\mu}}{\mu !} C_{\kappa}^{\mu}\left[\int_{0}^{t_{f}} d s \int_{0}^{t_{f}} d s^{\prime} \dot{j}^{T}(s) \cdot \mathfrak{D}\left(s-s^{\prime}\right) \cdot \boldsymbol{j}^{\prime}\left(s^{\prime}\right)\right]^{\mu-\kappa}\left[\boldsymbol{h}^{T} \cdot \int_{0}^{t_{f}} d s \dot{\boldsymbol{D}}_{2}\left(t_{f}-s\right) \cdot \boldsymbol{j}^{\prime}(s)\right]^{\kappa} .
$$

All the other terms in the binomial expansion are either of the order $O\left(j^{v+1}\right)$ or of the order $O\left(\boldsymbol{h}^{\kappa+1}\right)$. They will vanish after either performing the functional derivative $\delta^{v} / \delta j_{n_{1}}\left(s_{1}\right) \delta j_{n_{2}}\left(s_{2}\right) \cdots \delta j_{n_{v}}\left(s_{v}\right)$ and setting $\boldsymbol{j}=0$, or taking the derivative $\partial^{\kappa} / \partial h_{k_{1}} \partial h_{k_{2}} \cdots \partial h_{k_{\kappa}}$ and setting $\boldsymbol{h}=0$.

When the times in the functional derivative are the same, we have the following theorem

Theorem 3. For $\mu \geq 1$ and $\kappa<\mu$

$$
\left.\frac{\partial^{\kappa} \delta^{\nu+\mu} \boldsymbol{Z}_{\xi}^{(0)}\left[\boldsymbol{j}, \boldsymbol{j}^{\prime}, \boldsymbol{h}\right)}{\partial h_{k_{1}} \partial h_{k_{2}} \cdots \partial h_{k_{k}} \delta j_{n_{1}}(s) \delta j_{n_{2}}(s) \cdots \delta j_{n_{\nu}}(s) \delta j_{m_{1}}^{\prime}(s) \delta j_{m_{2}}^{\prime}(s) \cdots \delta j_{m_{\mu}}^{\prime}(s)}\right|_{j=j^{\prime}=\boldsymbol{h}=0}=0 .
$$

Proof. Again by counting the powers of $\boldsymbol{h}$ and $\boldsymbol{j}$ in the binomial expansion Eq. (D.3), we note that the non-vanishing terms will likely be

$$
\sum_{m=0}^{\mu} \frac{i^{\mu}}{\mu !} C_{m}^{\mu}\left[\int_{0}^{t_{f}} d s \int_{0}^{t_{f}} d s^{\prime} \boldsymbol{j}^{T}(s) \mathfrak{D}\left(s-s^{\prime}\right) \cdot \boldsymbol{j}^{\prime}\left(s^{\prime}\right)\right]^{\mu-m}\left[\boldsymbol{h}^{T} \cdot \int_{0}^{t_{f}} d s \dot{\boldsymbol{D}}_{2}\left(t_{f}-s\right) \cdot \boldsymbol{j}^{\prime}(s)\right]^{m},
$$

since the remaining terms in the expansion of (D.3) are of the order $\left(\boldsymbol{h}^{\kappa+1}\right)$, and they will vanish after taking the derivatives $\partial^{\kappa} / \partial h_{k_{1}} \partial h_{k_{2}} \cdots \partial h_{k_{\kappa}}$ and setting $\boldsymbol{h}=0$. Next we observe that $j_{n_{i}}$ and $j_{m_{i}}$ have the same time argument $s$. Thus when we perform the functional derivatives $\delta^{v} / \delta j_{n_{1}}(s) \delta j_{n_{2}}(s) \cdots \delta j_{n_{v}}(s)$ of Eq. (D.8), we will obtain terms proportional to $\mathfrak{D}(0)$, which by definition is zero.

Similarly, we have the following theorem

Theorem 4. For $\mu \geq 1$ and $\kappa+\lambda<\mu$, we have

$$
\left.\frac{\partial^{\kappa} \delta^{\lambda+\nu+\mu} \mathcal{Z}_{\xi}^{(0)}\left[\boldsymbol{j}, \boldsymbol{j}^{\prime}, \boldsymbol{h}\right)}{\partial h_{k_{1}} \partial h_{k_{2}} \cdots \partial h_{k_{k}} \delta j_{l_{1}}\left(t_{f}\right) \cdots \delta j_{l_{\lambda}}\left(t_{f}\right) \delta j_{n_{1}}(s) \delta j_{n_{2}}(s) \cdots \delta j_{n_{v}}(s) \delta j_{m_{1}}^{\prime}(s) \delta j_{m_{2}}^{\prime}(s) \cdots \delta j_{m_{\mu}}^{\prime}(s)}\right|_{j=j^{\prime}=\boldsymbol{h}=0}=0 .
$$

Proof. The proof is similar to Theorem 3. When we apply $\kappa j^{\prime}$-functional derivatives to the second pair of square brackets in (D.8) and $(\mu-\kappa) j^{\prime}$-functional derivatives to the first pair of square brackets in (D.8), if we would like to obtain a nonvanishing result in the limits $\boldsymbol{j}=\boldsymbol{j}^{\prime}=\boldsymbol{h}=0$, the only possibility is to apply $(\mu-\kappa) \boldsymbol{j}$-functional derivatives to the first pair of square brackets in (D.8) as well.

This means that we have to choose $(\mu-\kappa) \boldsymbol{j}$-functional derivatives among $\delta / \delta j_{l_{1}}\left(t_{f}\right), \cdots, \delta / \delta j_{l_{\lambda}}\left(t_{f}\right)$, and $\delta / \delta j_{n_{1}}(s)$, $\cdots, \delta / \delta j_{n_{v}}(s)$. Since $\mu-\kappa>\lambda$, this procedure unavoidably gives at a term proportional to $\mathfrak{D}(0)$, which by definition is zero. 
When all the functional derivatives are taken at the same time $t_{f}$, the following lemma guarantees that the functional derivatives in the late time limit $t_{f} \rightarrow \infty$ is independent of the initial state

Theorem 5 (independence of initial state). Given $\mathscr{Z}^{(0)}$ in (46) and the property of $\boldsymbol{D}_{i}$ in (C.15), we can shown

$$
\lim _{t_{f} \rightarrow \infty} \frac{\delta^{v} \mathscr{Z}^{(0)}\left[\varrho_{i}, j\right]}{\delta j_{n_{1}}\left(t_{f}\right) \delta j_{n_{2}}\left(t_{f}\right) \cdots \delta j_{n_{v}}\left(t_{f}\right)}=0 .
$$

Proof. We observe that for $v=1$, the proof is trivial

$$
\begin{aligned}
\frac{\delta \mathscr{Z}^{(0)}\left[\varrho_{i}, \boldsymbol{j}\right]}{\delta j_{n}\left(t_{f}\right)}=i \int d \boldsymbol{r}_{i}\left[\underline{\left.\boldsymbol{D}_{1}\left(t_{f}\right) \cdot \boldsymbol{r}_{i}\right]_{n} \times \exp \left\{i \int_{0}^{t_{f}} d s \boldsymbol{j}^{T}(s) \cdot \boldsymbol{D}_{1}(s) \cdot \boldsymbol{r}_{i}\right\} \varrho\left(\boldsymbol{r}_{i}, J\left(t_{f}\right)\right)}\right. \\
\quad+\int d \boldsymbol{r}_{i} \exp \left\{i \int_{0}^{t_{f}} d s \boldsymbol{j}^{T}(s) \cdot \boldsymbol{D}_{1}(s) \cdot \boldsymbol{r}_{i}\right\} \frac{\delta}{\delta J\left(t_{f}\right)} \varrho\left(\boldsymbol{r}_{i}, J\left(t_{f}\right)\right) \cdot \underline{\boldsymbol{D}}_{2}\left(t_{f}\right) \\
=0,
\end{aligned}
$$

where

$$
J\left(t_{f}\right)=\int_{0}^{t_{f}} d s \boldsymbol{D}_{2}(s) \cdot \boldsymbol{j}(s) .
$$

The vanishing result is the consequence of Eq. (C.15). Generalizing this observation straightforwardly to any integer $v$ concludes the proof

Another useful lemma is the following

Lemma 6. For signal $f(s)$ that has no singularity on $[0,+\infty)$ and decays as $s \rightarrow \infty$, i.e., $\lim _{s \rightarrow \infty}|f(s)|=0$, we then have

$$
\lim _{t_{f} \rightarrow \infty} \int_{0}^{t_{f}} d s D\left(t_{f}-s\right) f(s)=0,
$$

where $D(s)$ stands for any matrix element of the derivative or power of the fundamental solution $\boldsymbol{D}_{i}(s)$ discussed in Appendix $C$.

Proof. From the definition of the Laplace transform, we can easily see that the possible poles of $f(\mathfrak{s})$ must lie on the left half of the complex $\mathfrak{s}$ plane. Take $D(s)$ be the matrix element of $\boldsymbol{D}_{i}(s)$, and then by the Laplace convolution theorem, we obtain

$$
\begin{aligned}
\lim _{t_{f} \rightarrow \infty} \int_{0}^{t_{f}} d s\left[\boldsymbol{D}_{i}\left(t_{f}-s\right)\right]_{j k} f(s) & =\lim _{t_{f} \rightarrow \infty} \frac{1}{2 \pi i} \int_{\operatorname{Re} \mathfrak{s}_{0}-i \infty}^{\operatorname{Re} \mathfrak{s}_{0}+i \infty} d \mathfrak{s}\left[\boldsymbol{D}_{i}(\mathfrak{s})\right]_{j k} f(\mathfrak{s}) e^{\mathfrak{s} t_{f}} \\
& =\lim _{t_{f} \rightarrow \infty} \frac{1}{2 \pi i} \int_{\operatorname{Re} \mathfrak{s}_{0}-i \infty}^{\operatorname{Re} \mathfrak{s}_{0}+i \infty} d \mathfrak{s} \frac{\left[\boldsymbol{W}_{i}(\mathfrak{s})\right]_{j k} f(\mathfrak{s}) e^{\mathfrak{s} t_{f}}}{\prod_{k=1}^{4}\left(\mathfrak{s}-\mathfrak{s}_{k}\right)}
\end{aligned}
$$

where $\operatorname{Re} \mathfrak{s}_{0}$ is a real number so that the integration is well defined. Since all of the poles $\mathfrak{s}_{m}$ of the integrand lie on the left half of the complex $\mathfrak{s}$ plane, i.e., $\operatorname{Re} \mathfrak{s}_{m}<0$, the factor $e^{t_{f}} \operatorname{Re} \mathfrak{s}_{m}$ will approach zero exponentially in the limit $t_{f} \rightarrow \infty$. Thus from the residue theorem, we conclude that (D.14) will vanish in the same limit. Since any powers or times derivatives of $\boldsymbol{D}_{i}(\mathfrak{s})$ have the same singular structure as $\boldsymbol{D}_{i}(\mathfrak{s})$, the conclusion naturally extends to the case that $D(s)$ is powers of time derivatives of any matrix element of $\boldsymbol{D}_{i}(s)$.

When we apply Theorem 6 in what follows, the function $f$ may contain the Hadamard Green's function defined by Eq. (A.5), which is divergent without regularization. This will render $f(s)$ no longer analytic on the $s \in[0, \infty)$ or at $s=\infty$. However, realistically there is always some regularization imposed by physical constraint on the Hadamard Green's function, such as the cutoff regularization, which will make it convergent. Therefore, the Hadamard Green's function in what follows should be understood as the regularized one and then we could apply Theorem 6 without any further concerns. 


\section{Theorem 7.}

$$
\begin{aligned}
& \left.\lim _{t_{f} \rightarrow \infty} \int_{0}^{t_{f}} d s\left\langle\left[\dot{\boldsymbol{D}}_{2}\left(t_{f}-s\right)\right]_{n r} \xi_{n}\left(t_{f}\right) \frac{\delta^{3} \mathscr{Z}^{(0)}\left[\varrho_{i}, \boldsymbol{j}\right] \exp \left\{i \overline{\mathscr{S}}_{\xi}^{(0)}[\boldsymbol{j}, \boldsymbol{h})\right\}}{\delta j_{k}(s) \delta j_{l}(s) \delta j_{m}(s)}\right\rangle\right|_{\boldsymbol{j}=\boldsymbol{h}=0} \\
= & \left.\lim _{t_{f} \rightarrow \infty} \int_{0}^{t_{f}} d s\left\langle\left[\dot{\boldsymbol{D}}_{2}\left(t_{f}-s\right)\right]_{n r} \xi_{n}\left(t_{f}\right) \frac{\delta^{3} \exp \left\{i \overline{\mathscr{S}}_{\xi}^{(0)}[\boldsymbol{j}, \boldsymbol{h})\right\}}{\delta j_{k}(s) \delta j_{l}(s) \delta j_{m}(s)}\right\rangle\right|_{\boldsymbol{j}=\boldsymbol{h}=0} .
\end{aligned}
$$

Proof. In order to generate a non-vanishing results after averaging on all the realization of $\boldsymbol{\xi}$, we keep terms that are even powers in $\xi$. Thus either one or all of the functional derivatives among $\delta / \delta j_{k}(s), \delta / \delta j_{l}(s), \delta / \delta j_{m}(s)$ should be applied to $\exp \left\{i \overline{\mathscr{S}}_{\xi}^{(0)}[\boldsymbol{j}, \boldsymbol{h})\right\}$. Now we show that the former case will give vanishing values.

Suppose we apply $\delta / \delta j_{k}(s)$ to $\exp \left\{i \overline{\mathscr{S}}_{\xi}^{(0)}[\boldsymbol{j}, \boldsymbol{h})\right\}$, and $\delta / \delta j_{l}(s), \delta / \delta j_{m}(s)$ to $\mathscr{Z}^{(0)}\left[\varrho_{i}, \boldsymbol{j}\right]$. Then after taking the ensemble average, we obtain expressions like

$$
\begin{aligned}
& \int_{0}^{t_{f}} d s \int_{0}^{t_{f}} d s^{\prime}\left[\boldsymbol{D}_{2}\left(t_{f}-s\right)\right]_{n r}\left[\mathfrak{D}\left(s-s^{\prime}\right)\right]_{k p}\left\langle\left\langle\xi_{n}\left(t_{f}\right) \xi_{p}\left(s^{\prime}\right)\right\rangle\right\rangle\left[\boldsymbol{D}_{i}(s)\right]_{l \alpha}\left[\boldsymbol{D}_{j}(s)\right]_{m \beta} \\
\sim \epsilon_{1}\left(t_{f}\right) \equiv & \int_{0}^{t_{f}} d s \int_{0}^{t_{f}} d s^{\prime} D\left(t_{f}-s\right) \mathcal{D}\left(s-s^{\prime}\right) G_{H}\left(t_{f}-s^{\prime}\right) D^{2}(s),
\end{aligned}
$$

where $\left[\boldsymbol{D}_{i}(s)\right]_{l \alpha}$ and $\left[\boldsymbol{D}_{j}(s)\right]_{m \beta}$ result from the application of $\delta / \delta j_{l}(s), \delta / \delta j_{m}(s)$ on $\mathscr{Z}^{(0)}\left[\varrho_{i}, \boldsymbol{j}\right]$. Now we make the change of variables $t_{f}-s \rightarrow s, t_{f}-s^{\prime} \rightarrow s^{\prime}$, and then Eq. (D.16) becomes

$$
\epsilon_{1}\left(t_{f}\right)=\int_{0}^{t_{f}} d s D^{2}\left(t_{f}-s\right)\left[\int_{s}^{t_{f}} d s^{\prime} D(s) D\left(s^{\prime}-s\right) G_{H}\left(s^{\prime}\right)\right] .
$$

Since both $D(s)$ and $G_{H}(s)$ vanish at $s=\infty$, we know $\lim _{t_{f} \rightarrow \infty} \int_{s}^{t_{f}} d s^{\prime} D(s) D\left(s^{\prime}-s\right) G_{H}\left(s^{\prime}\right)$ exists and we can safely set $t_{f}=\infty$

$$
\lim _{t_{f} \rightarrow \infty} \int_{s}^{t_{f}} d s^{\prime} D(s) D\left(s^{\prime}-s\right) G_{H}\left(s^{\prime}\right)=\int_{s}^{\infty} d s^{\prime} D(s) D\left(s^{\prime}-s\right) G_{H}\left(s^{\prime}\right) .
$$

Therefore in the large $t_{f}$ limit, we find $\epsilon_{1}\left(t_{f}\right)$ given by

$$
\lim _{t_{f} \rightarrow \infty} \epsilon_{1}\left(t_{f}\right)=\lim _{t_{f} \rightarrow \infty} \int_{0}^{t_{f}} d s D^{2}\left(t_{f}-s\right)\left[\int_{s}^{\infty} d s^{\prime} D(s) D\left(s^{\prime}-s\right) G_{H}\left(s^{\prime}\right)\right] .
$$

Since

$$
\lim _{s \rightarrow \infty} \int_{s}^{\infty} d s^{\prime} D(s) D\left(s^{\prime}-s\right) G_{H}\left(s^{\prime}\right)=0
$$

by Lemma 6, we have

$$
\lim _{t_{f} \rightarrow \infty} \epsilon_{1}\left(t_{f}\right)=0
$$

In the second case, all the functional derivatives $\delta / \delta j_{k}(s), \delta / \delta j_{l}(s), \delta / \delta j_{p}(s)$ apply to $\exp \left\{i \overline{\mathscr{S}}_{\xi}^{(0)}[\boldsymbol{j}, \boldsymbol{h})\right\}$, and $\mathscr{Z}^{(0)}\left[\varrho_{i}, \boldsymbol{j}=0\right]=1$, so this gives (D.15). This concludes the proof.

By similar procedures, because $\boldsymbol{\xi}$ has a Gaussian distribution, we can readily show

\section{Theorem 8.}

$$
\begin{aligned}
& \left.\lim _{t_{f} \rightarrow \infty} \int_{0}^{t_{f}} d s\left\langle\left[\dot{\boldsymbol{D}}_{2}\left(t_{f}-s\right)\right]_{n r} \xi_{n}\left(t_{f}\right) \frac{\delta^{2} \mathscr{Z}^{(0)}\left[\varrho_{i}, \boldsymbol{j}\right] \exp \left\{i \overline{\mathscr{S}}_{\xi}^{(0)}[\boldsymbol{j}, \boldsymbol{h})\right\}}{\delta j_{k}(s) \delta j_{l}(s)}\right\rangle\right|_{\boldsymbol{j}=\boldsymbol{h}=0} \\
= & \left.\lim _{t_{f} \rightarrow \infty} \int_{0}^{t_{f}} d s\left\langle\left[\dot{\boldsymbol{D}}_{2}\left(t_{f}-s\right)\right]_{n r} \xi_{n}\left(t_{f}\right) \frac{\delta^{2} \exp \left\{i \overline{\mathscr{S}}_{\xi}^{(0)}[\boldsymbol{j}, \boldsymbol{h})\right\}}{\delta j_{k}(s) \delta j_{l}(s)}\right\rangle\right|_{\boldsymbol{j}=\boldsymbol{h}=0} \\
= & 0 .
\end{aligned}
$$


Similar conclusions can be further generalized. We prove the following theorems with the assistance of Lemma 6.

\section{Theorem 9.}

$$
\begin{aligned}
& \left.\lim _{t_{f} \rightarrow \infty} \int_{0}^{t_{f}} d s \int_{0}^{t_{f}} d s^{\prime}\left\langle\left[\dot{\boldsymbol{D}}_{2}\left(t_{f}-s\right)\right]_{n r}\left[\dot{\boldsymbol{D}}_{2}\left(t_{f}-s^{\prime}\right)\right]_{n p} \xi_{p}\left(s^{\prime}\right) \frac{\delta^{3} \mathscr{Z}^{(0)}\left[\varrho_{i}, \boldsymbol{j}\right] \exp \left\{i \overline{\mathscr{S}}_{\xi}^{(0)}[\boldsymbol{j}, \boldsymbol{h})\right\}}{\delta j_{k}(s) \delta j_{l}(s) \delta j_{m}(s)}\right\rangle\right|_{\boldsymbol{j}=\boldsymbol{h}=0} \\
= & \left.\lim _{t_{f} \rightarrow \infty} \int_{0}^{t_{f}} d s \int_{0}^{t_{f}} d s^{\prime}\left\langle\left[\dot{\boldsymbol{D}}_{2}\left(t_{f}-s\right)\right]_{n r}\left[\dot{\boldsymbol{D}}_{2}\left(t_{f}-s^{\prime}\right)\right]_{n p} \xi_{p}\left(s^{\prime}\right) \frac{\delta^{3} \exp \left\{i \overline{\mathscr{S}}_{\xi}^{(0)}[\boldsymbol{j}, \boldsymbol{h})\right\}}{\delta j_{k}(s) \delta j_{l}(s) \delta j_{m}(s)}\right\rangle\right|_{\boldsymbol{j}=\boldsymbol{h}=0} .
\end{aligned}
$$

Proof. In order to obtain a non-vanishing results, we keep terms that are even powers in $\boldsymbol{\xi}$. Thus, either one or all of the three functional derivatives can be applied to $\exp \left\{i \overline{\mathscr{S}}_{\xi}^{(0)}[\boldsymbol{j}, \boldsymbol{h}=0)\right\}$. For the former case, let us apply, say, $\delta / \delta j_{k}(s)$ to $\exp \left\{i \overline{\mathscr{S}}_{\xi}^{(0)}[\boldsymbol{j}, \boldsymbol{h}=0)\right\}$ and $\delta / \delta j_{l}(s), \delta / \delta j_{m}(s)$ to $\mathscr{Z}^{(0)}\left[\varrho_{i}, \boldsymbol{j}\right]$.

Then we will have expressions like

$$
\begin{gathered}
\int_{0}^{t_{f}} d s \int_{0}^{t_{f}} d s^{\prime} \int_{0}^{t_{f}} d s_{1}\left[\dot{\boldsymbol{D}}_{2}\left(t_{f}-s\right)\right]_{n r}\left[\dot{\boldsymbol{D}}_{2}\left(t_{f}-s^{\prime}\right)\right]_{n p}\left[\mathfrak{D}\left(s-s_{1}\right)\right]_{k q}\left\langle\left\langle\xi_{p}\left(s^{\prime}\right) \xi_{q}\left(s_{1}\right)\right\rangle\right\rangle\left[\boldsymbol{D}_{i}(s)\right]_{l \alpha}\left[\boldsymbol{D}_{j}(s)\right]_{m \beta} \\
\sim \epsilon_{2}\left(t_{f}\right) \equiv \int_{0}^{t_{f}} d s \dot{D}\left(t_{f}-s\right)\left[D^{2}(s) \int_{0}^{t_{f}} d s^{\prime} \int_{0}^{s} d s_{1} D\left(s-s_{1}\right) G_{H}\left(s^{\prime}-s_{1}\right)\right] .
\end{gathered}
$$

Again since $D(s)$ and $G_{H}(s)$ vanish at $s=\infty$, so we find

$$
\left|\lim _{t_{f} \rightarrow \infty} \int_{0}^{t_{f}} d s^{\prime} \int_{0}^{s} d s_{1} D\left(s-s_{1}\right) G_{H}\left(s^{\prime}-s_{1}\right)\right|=\left|\int_{0}^{\infty} d s^{\prime} \int_{0}^{s} d s_{1} D\left(s-s_{1}\right) G_{H}\left(s^{\prime}-s_{1}\right)\right|<\infty .
$$

Thus we obtain

$$
\lim _{t_{f} \rightarrow \infty} \epsilon_{2}\left(t_{f}\right)=\lim _{t_{f} \rightarrow \infty} \int_{0}^{t_{f}} d s \dot{D}\left(t_{f}-s\right)\left[D^{2}(s) \int_{0}^{\infty} d s^{\prime} \int_{0}^{s} d s_{1} D\left(s-s_{1}\right) G_{H}\left(s^{\prime}-s_{1}\right)\right] .
$$

On the other hand, we know $D^{2}(s)$ decays as $s \rightarrow \infty$ and

$$
\lim _{s \rightarrow \infty}\left|\int_{0}^{\infty} d s^{\prime} \int_{0}^{s} d s_{1} D\left(s-s_{1}\right) G_{H}\left(s^{\prime}-s_{1}\right)\right|<\infty,
$$

so we conclude

$$
\lim _{s \rightarrow \infty}\left[D^{2}(s) \int_{0}^{t_{f}} d s^{\prime} \int_{0}^{s} d s_{1} D\left(s-s_{1}\right) G_{H}\left(s^{\prime}-s_{1}\right)\right]=0
$$

By Lemma 6, we obtain

$$
\lim _{t_{f} \rightarrow \infty} \epsilon_{2}\left(t_{f}\right)=0
$$

\section{Theorem 10.}

$$
\begin{aligned}
& \left.\lim _{t_{f} \rightarrow \infty} \int_{0}^{t_{f}} d s \int_{0}^{t_{f}} d s^{\prime}\left\langle\left[\dot{\boldsymbol{D}}_{2}\left(t_{f}-s\right)\right]_{n r}\left[\dot{\boldsymbol{D}}_{2}\left(t_{f}-s^{\prime}\right)\right]_{n p} \xi_{p}\left(s^{\prime}\right) \frac{\delta^{2} \mathscr{Z}(0)\left[\varrho_{i}, \boldsymbol{j}\right] \exp \left\{i \overline{\mathcal{S}}_{\xi}^{(0)}[\boldsymbol{j}, \boldsymbol{h})\right\}}{\delta j_{k}(s) \delta j_{l}(s)}\right\rangle\right|_{\boldsymbol{j}=\boldsymbol{h}=0} \\
= & \left.\lim _{t_{f} \rightarrow \infty} \int_{0}^{t_{f}} d s \int_{0}^{t_{f}} d s^{\prime}\left\langle\left[\dot{\boldsymbol{D}}_{2}\left(t_{f}-s\right)\right]_{n r}\left[\dot{\boldsymbol{D}}_{2}\left(t_{f}-s^{\prime}\right)\right]_{n p} \xi_{p}\left(s^{\prime}\right) \frac{\delta^{2} \exp \left\{i \overline{\mathcal{S}}_{\xi}^{(0)}[\boldsymbol{j}, \boldsymbol{h})\right\}}{\delta j_{k}(s) \delta j_{l}(s)}\right\rangle\right|_{\boldsymbol{j}=\boldsymbol{h}=0} \\
= & 0
\end{aligned}
$$




\section{Theorem 11.}

$$
\begin{aligned}
& \left.\lim _{t_{f} \rightarrow \infty} \int_{0}^{t_{f}} d s \int_{0}^{t_{f}} d s^{\prime}\left\langle\left[\dot{\boldsymbol{D}}_{2}\left(t_{f}-s\right)\right]_{n r}\left[\dot{\boldsymbol{D}}_{2}\left(t_{f}-s^{\prime}\right)\right]_{n p} \frac{\delta \mathscr{Z}^{(0)}\left[\varrho_{i}, \boldsymbol{j}\right] \exp \left\{i \overline{\mathcal{S}}_{\xi}^{(0)}[\boldsymbol{j}, \boldsymbol{h})\right\}}{\delta j_{k}(s)}\right\rangle\right|_{\boldsymbol{j}=\boldsymbol{h}=0} \\
= & \left.\lim _{t_{f} \rightarrow \infty} \int_{0}^{t_{f}} d s \int_{0}^{t_{f}} d s^{\prime}\left\langle\left[\dot{\boldsymbol{D}}_{2}\left(t_{f}-s\right)\right]_{n r}\left[\dot{\boldsymbol{D}}_{2}\left(t_{f}-s^{\prime}\right)\right]_{n p} \frac{\delta \exp \left\{i \overline{\mathcal{S}}_{\xi}^{(0)}[\boldsymbol{j}, \boldsymbol{h})\right\}}{\delta j_{k}(s)}\right\rangle\right|_{\boldsymbol{j}=\boldsymbol{h}=0} \\
= & 0 .
\end{aligned}
$$

Theorems 7-11 imply that we can set $\mathscr{Z}^{(0)}\left[\varrho_{i}, j\right]$ in Eq. (45) to unity, which is equivalent to taking $\boldsymbol{j}=0$ in $\mathscr{Z}^{(0)}\left[\varrho_{i}, j\right]$, without changing the heat transport in the late time limit. Since the dependence of the initial state of the chain is included in $\mathscr{Z}^{(0)}\left[\varrho_{i}, j\right]$, it then implies that these late-time results are independent of the initial configurations of the chain.

\section{Appendix E. Useful identities for proving the NESS at the first order}

In this appendix, we will construct the identities useful for proving the NESS at the first order in nonlinearity in Sec. 6.2.

It will be convenient for the following discussions if we decompose the right hand side of Eq. (125) into three components and denote them by

$$
\Gamma_{k l m}^{n r}={ }_{\mathrm{I}} \Gamma_{k l m}^{n r}+{ }_{\mathbb{I}} \Gamma_{k l m}^{n r}+{ }_{\mathbb{I I}} \Gamma_{k l m}^{n r},
$$

and similar decompositions will be applied to $\tilde{\Gamma}_{k l m}^{n r}, \Upsilon_{v k l m}^{n r}, \tilde{\Upsilon}_{v k l m}^{n r}$ and $\Lambda_{k l m}^{n}$. as well. Furthermore, we note that at late time the correlation matrix $\mathbf{C}$ in the coincident limit actually takes the form

$$
\boldsymbol{C}_{l m}=\int_{-\infty}^{\infty} \frac{d \omega}{2 \pi}\left[\tilde{\mathfrak{D}}(\omega) \tilde{\boldsymbol{G}}_{H}(\omega) \tilde{\mathfrak{D}}(-\omega)\right]_{l m} .
$$

With the help of the properties of $\tilde{D}(\omega)$ outlined in Eqs. (117-119), we find

$$
\begin{aligned}
\boldsymbol{C}_{l m} & =\sum_{k=1}^{2} \int_{-\infty}^{\infty} \frac{d \omega}{2 \pi}[\tilde{\mathfrak{D}}(\omega)]_{l k}\left[\tilde{\mathfrak{D}}^{*}(\omega)\right]_{k m}\left[\tilde{\boldsymbol{G}}_{H}(\omega)\right]_{k k} \\
{ }_{\mathrm{I}} \Gamma_{k l m}^{n r} & \left.=-\boldsymbol{C}_{l m} \int_{-\infty}^{\infty} \frac{d \omega}{2 \pi} \omega \operatorname{Im}\left([\tilde{\mathfrak{D}}(\omega)]_{n r} \tilde{\mathfrak{D}}(\omega)\right]_{n k}\right)\left[\tilde{\boldsymbol{G}}_{H}(\omega)\right]_{n n} \\
{ }_{\mathrm{I}} \tilde{\Gamma}_{k l m}^{n r} & =2 \boldsymbol{C}_{l m} \sum_{p=1}^{2} \int_{-\infty}^{\infty} \frac{d \omega}{2 \pi} \omega^{2} \operatorname{Re}\left([\tilde{\mathfrak{D}}(\omega)]_{n r}[\tilde{\mathfrak{D}}(\omega)]_{n p}^{*}[\tilde{\mathfrak{D}}(\omega)]_{p k}\right)\left[\tilde{\boldsymbol{G}}_{H}(\omega)\right]_{p p} \\
{ }_{\mathrm{I}} \Upsilon_{v k l m}^{n r} & =-\boldsymbol{C}_{l m} \sum_{p=1}^{2} \int_{-\infty}^{\infty} \frac{d \omega}{2 \pi} \omega \operatorname{Im}\left([\tilde{\mathfrak{D}}(\omega)]_{n r}[\tilde{\mathfrak{D}}(\omega)]_{v p}^{*}[\tilde{\mathfrak{D}}(\omega)]_{p k}\right)\left[\tilde{\boldsymbol{G}}_{H}(\omega)\right]_{p p} \\
{ }_{\mathrm{I}} \tilde{\Upsilon}_{v k l m}^{n r} & =\boldsymbol{C}_{l m} \sum_{p=1}^{2} \int_{-\infty}^{\infty} \frac{d \omega}{2 \pi} \omega \operatorname{Im}\left([\tilde{\mathfrak{D}}(\omega)]_{v r}[\tilde{\mathfrak{D}}(\omega)]_{n p}^{*}[\tilde{\mathfrak{D}}(\omega)]_{p k}\right)\left[\tilde{\boldsymbol{G}}_{H}(\omega)\right]_{p p} \\
{ }_{\mathrm{I}} \Lambda_{k l m}^{n} & =-\boldsymbol{C}_{l m} \sum_{p=1}^{2} \int_{-\infty}^{\infty} \frac{d \omega}{2 \pi} \omega \operatorname{Im}\left([\tilde{\mathfrak{D}}(\omega)]_{n p}\left[\tilde{\mathfrak{D}}^{*}(\omega)\right]_{p k}\right)\left[\tilde{\boldsymbol{G}}_{H}(\omega)\right]_{p p}
\end{aligned}
$$

so we arrive at

$$
-{ }_{\mathrm{I}} \Gamma_{k l m}^{n r}+2 \gamma_{\mathrm{I}} \tilde{\Gamma}_{k l m}^{n r}-\lambda_{2}\left({ }_{\mathrm{I}} \Upsilon^{n k l m}+{ }_{\mathrm{I}} \tilde{\Upsilon}_{v k l m}^{n r}\right)=\boldsymbol{C}_{l m} \int_{-\infty}^{\infty} \frac{d \omega}{2 \pi}\left\{\mathcal{K}_{v k}^{n r}\left[\tilde{\boldsymbol{G}}_{H}(\omega)\right]_{n n}+\mathcal{L}_{v k}^{n r}(\omega)\left[\tilde{\boldsymbol{G}}_{H}(\omega)\right]_{v v}\right\},
$$


where

$$
\begin{aligned}
\mathcal{K}_{v k}^{n r}(\omega) & \left.\equiv \omega \operatorname{Im}\left([\tilde{\mathfrak{D}}(\omega)]_{n r} \tilde{\mathfrak{D}}(\omega)\right]_{n k}\right)+4 \gamma \omega^{2} \operatorname{Re}\left([\tilde{\mathfrak{D}}(\omega)]_{n r}[\tilde{\mathfrak{D}}(\omega)]_{n n}^{*}[\tilde{\mathfrak{D}}(\omega)]_{n k}\right) \\
& +\omega\left[\lambda_{2} \operatorname{Im}\left([\tilde{\mathfrak{D}}(\omega)]_{n r}[\tilde{\mathfrak{D}}(\omega)]_{v n}^{*}[\tilde{\mathfrak{D}}(\omega)]_{n k}\right)-\operatorname{Im}\left([\tilde{\mathfrak{D}}(\omega)]_{v r}[\tilde{\mathfrak{D}}(\omega)]_{n n}^{*}[\tilde{\mathfrak{D}}(\omega)]_{n k}\right)\right] \\
\mathcal{L}_{v k}^{n r}(\omega) & \left.\equiv 4 \gamma \omega^{2} \operatorname{Re}\left([\tilde{\mathfrak{D}}(\omega)]_{n r}[\tilde{\mathfrak{D}}(\omega)]_{n v}^{*} \tilde{\mathfrak{D}}(\omega)\right]_{v k}\right)+ \\
& +\omega \lambda_{2}\left[\operatorname{Im}\left([\tilde{\mathfrak{D}}(\omega)]_{n r}[\tilde{\mathfrak{D}}(\omega)]_{v v}^{*}[\tilde{\mathfrak{D}}(\omega)]_{v k}\right)-\operatorname{Im}\left([\tilde{\mathfrak{D}}(\omega)]_{v r}[\tilde{\mathfrak{D}}(\omega)]_{n v}^{*}[\tilde{\mathfrak{D}}(\omega)]_{v k}\right)\right]
\end{aligned}
$$

Let us now using Eqs. (115-118) to simplify $\mathcal{K}_{v k}^{n r}(\omega)$ and $\mathcal{L}_{v k}^{n r}(\omega)$. Eliminate [ $\left.\tilde{\mathfrak{D}}(\omega)\right]_{v n}^{*}$ in Eq. (E.9) with Eq. (117), we obtain

$$
\begin{aligned}
\mathcal{K}_{v k}^{n r}(\omega) & =\omega \operatorname{Im}\left\{\left(-\omega^{2}+2 i \gamma \omega+\omega_{R 0}^{2}+\lambda_{2}\right)[\tilde{\mathfrak{D}}(\omega)]_{n r}[\tilde{\mathfrak{D}}(\omega)]_{n n}^{*}[\tilde{\mathfrak{D}}(\omega)]_{n k}\right\} \\
& -\omega \lambda_{2} \operatorname{Im}\left([\tilde{\mathfrak{D}}(\omega)]_{v r}[\tilde{\mathfrak{D}}(\omega)]_{n n}^{*}[\tilde{\mathfrak{D}}(\omega)]_{n k}\right)
\end{aligned}
$$

Upon using Eq. (117) for $r=n$ and Eq. (118) for $r=v$, one immediately obtains

$$
\begin{aligned}
& \mathcal{K}_{v k}^{n n}(\omega)=\omega \lambda_{2} \operatorname{Im}\left([\tilde{\mathfrak{D}}(\omega)]_{n n}^{*}[\tilde{\mathfrak{D}}(\omega)]_{n k}\right) \\
& \mathcal{K}_{v k}^{n v}(\omega)=0
\end{aligned}
$$

Similarly, eliminating $[\tilde{\mathfrak{D}}(\omega)]_{v v}^{*}$ in Eq. (E.10) with Eq. (118) yields

$$
\begin{aligned}
\mathcal{L}_{v k}^{n r}(\omega) & =\omega \operatorname{Im}\left\{\left(-\omega^{2}+2 i \gamma \omega+\omega_{R 0}^{2}+\lambda_{2}\right)[\tilde{\mathfrak{D}}(\omega)]_{n r}[\tilde{\mathfrak{D}}(\omega)]_{n v}^{*}[\tilde{\mathfrak{D}}(\omega)]_{v k}\right\} \\
& -\omega \lambda_{2} \operatorname{Im}\left([\tilde{\mathfrak{D}}(\omega)]_{v r}[\tilde{\mathfrak{D}}(\omega)]_{n v}^{*}[\tilde{\mathfrak{D}}(\omega)]_{v k}\right)
\end{aligned}
$$

Then again using Eq. (117) for $r=n$ and Eq. (118) for $r=v$, one obtain

$$
\begin{aligned}
& \mathcal{L}_{v k}^{n n}(\omega)=\omega \operatorname{Im}\left([\tilde{\mathfrak{D}}(\omega)]_{n v}^{*}[\tilde{\mathfrak{D}}(\omega)]_{v k}\right) \\
& \mathcal{L}_{v k}^{n v}(\omega)=0
\end{aligned}
$$

Now from Eqs. (E.12, E.13, E.15, E.16), one immediately obtains

$$
\begin{aligned}
& -{ }_{\mathrm{I}} \Gamma_{n n n}^{n n}+2 \gamma_{\mathrm{I}} \tilde{\Gamma}_{n n n}^{n n}-\lambda_{2}\left({ }_{\mathrm{I}} \Upsilon^{n n n n}+{ }_{\mathrm{I}} \tilde{\Upsilon}_{v n n n}^{n n}\right)=0 \\
& -{ }_{\mathrm{I}} \Gamma_{k l m}^{n v}+2 \gamma_{\mathrm{I}} \tilde{\Gamma}_{k l m}^{n v}-\lambda_{2}\left({ }_{\mathrm{I}} \Upsilon_{v k l m}^{n v}+{ }_{\mathrm{I}} \tilde{\Upsilon}_{v k l m}^{n v}\right)=0
\end{aligned}
$$

Furthermore, Eqs. (E.12, E.13, E.15, E.16) also indicate that

$$
-{ }_{\mathrm{I}} \Gamma_{v l m}^{n n}+2 \gamma_{\mathrm{I}} \tilde{\Gamma}_{v l m}^{n n}-\lambda_{2}\left({ }_{\mathrm{I}} \Upsilon_{v v l m}^{n n}+{ }_{\mathrm{I}} \tilde{\Upsilon}_{v v l m}^{n n}\right)={ }_{\mathrm{I}} \Lambda_{v l m}^{n}
$$

The procedures work for cases II and III because they differ by permutations of the indices, so Eqs. (E.17)-(E.19) hold in general without the left subscripts I, II and III. Therefore, we see that Eq. (E.17) leads to Eq. (144) while Eq. (E.18)leads to Eqs. (145-153). Finally, Eq. (E.19) proves Eqs. (154-155).

\section{References}

[1] G. Gallavotti and E. G. D. Cohen, "Dynamical ensembles in nonequilibrium statistical mechanics", Phys. Rev. Lett. 74, 2694 (1995).

[2] D. J. Evans, E. G. D. Cohen and G. P. Morriss, "Probability of second law violations in shearing steady states", Phys. Rev. Lett. 71, 2401 (1993);

D. J. Evans and D. J. Searles, "Equilibrium microstates which generate second law violating steady states", Phys. Rev. E 50, 1645 (1994);

D. J. Evans and D. J. Searles, "The fluctuation theorem", Adv. Phys. 51, 1529 (2002).

[3] J. Kurchan, "Fluctuation theorem for stochastic dynamics", J. Phys. A 31, 3719 (1998).

[4] J. L. Lebowitz and H. Spohn, "A Gallavotti-Cohen-Type symmetry in the large deviation functional for stochastic dynamics", J. Stat. Phys. 95, 333 (1999).

[5] U. Seifert, "Stochastic thermodynamics, fluctuation theorems and molecular machines", Rep. Prog. Phys. 75, 126001, (2012). 
[6] C. Jarzynski, "Nonequilibrium equality for free energy differences", Phys. Rev. Lett. 78, 2690 (1997);

C. Jarzynski, "Equilibrium free-energy differences from nonequilibrium measurements: a master-equation approach", Phys. Rev. E 56, 5018 (1997).

[7] G. E. Crooks, "Entropy production fluctuation theorem and the nonequilibrium work relation for free energy differences", Phys. Rev. E 60, 2721 (1999).

[8] M. Esposito, U. Harbola and S. Mukamel, "Nonequilibrium fluctuations, fluctuation theorems, and counting statistics in quantum systems", Rev. Mod. Phys. 81, 1665 (2009);

M. Campisi, P. Hänggi and P. Talkner, “Quantum fluctuation relations: foundations and applications”, Rev. Mod. Phys. 83, 771 (2011).

[9] C. Jarzynski, "Equalities and inequalities: irreversibility and the second law of thermodynamics at the nanoscale", Ann. Rev. Cond. Mat. Phys. 2, 329 (2011).

[10] Y. Subaşi and B. L. Hu, "Quantum and classical fluctuation theorems from a decoherent-histories open-system analysis", Phys. Rev. E 85, $011112(2012)$.

[11] J. Gemmer, M. Michel and G. Mahler, "Quantum Thermodynamics - Emergence of Thermodynamic Behavior within Composite Quantum Systems", 2nd Edition (Springer Verlag, Berlin, 2004).

[12] R. Kosloff, "Quantum thermodynamics: a dynamical viewpoint”, Entropy 15, 2100 (2013).

[13] S. Vinjanampathy and J. Anders, "Quantum thermodynamics", Contemp Phys. 57, 545 (2016).

[14] S. Deffner and S. Campbell, "Quantum Thermodynamics: An Introduction to the Thermodynamics of Quantum Information", (Morgan \& Claypool, 2019); [arXiv:1907.01596]

[15] Z. Rieder, J. L. Lebowitz and E. Lieb, "Properties of a harmonic crystal in a stationary non-equilibrium state", J. Math. Phys. 8, 1073 (1967); A. Casher and J. L. Lebowitz, "Heat flow in regular and disordered harmonic chains", J. Math. Phys. 12, 1701 (1971);

A. J. O'Connor and J. L. Lebowitz, "Heat conduction and sound transmission in isotopically disordered harmonic crystals", J. Math. Phys. 15, 692 (1974);

H. Spohn and J. L. Lebowitz, "Stationary non-equilibrium states of infinite harmonic systems”, Commun. Math. Phys. 54, 97 (1977).

[16] J.-P. Eckmann, C.-A. Pillet and L. Rey-Bellet, "Non-equilibrium statistical mechanics of anharmonic chains coupled to two heat baths at different temperatures", Commun. Math. Phys. 201, 657 (1999);

J.-P. Eckmann and M. Hairer, "Non-equilibrium statistical mechanics of strongly anharmonic chains of oscillators", Commun. Math. Phys. 212, 105 (2000);

L. Rey-Bellet and L. E. Thomas, "Exponential convergence to non-equilibrium stationary states in classical statistical mechanics", Commun. Math. Phys. 225, 305 (2002).

[17] J.-T. Hsiang and B. L. Hu, "Nonequilibrium steady state in open quantum systems: influence action, stochastic equation and power balance", Ann. of Phys. 362, 139 (2015).

[18] T. Motz, J. Ankerhold and J. T. Stockburger, "Currents and fluctuations of quantum heat transport in harmonic chains", New J. Phys. 19 053013 (2017);

T. Motz, M. Wiedmann, J. T. Stockburger and J. Ankerhold, "Rectification of heat currents across nonlinear quantum chains: a versatile approach beyond weak thermal contact", New J. Phys. 20, 113020 (2018).

[19] E. Fermi, J. Pasta and S. Ulam, "Studies of nonlinear problems", Los Alamos report, LA-1940 (1955).

[20] D. K. Campbell, P. Rosenau and G. Zaslavsky, "Introduction: the Fermi-Pasta-Ulam problem: the first fifty years", Chaos 1, 015101 (2005); G. Gallavotti, "The Fermi-Pasta-Ulam Problem: A Status Report”, Lecture Notes in Physics, Vol. 728, (Springer Verlag, Berlin, 2008).

[21] F. T. Hioe and E. W. Montroll, "Quantum theory of anharmonic oscillators. I. Energy levels of oscillators with positive quartic anharmonicity", J. Math. Phys. 16, 1945 (1975).

[22] C. M. Bender and S. Boettcher, "Quasi-exactly solvable quartic potential", J. Phys. A 31, L273 (1998).

[23] B. L. Hu, Lectures at the Seventh International Latin-American Symposium on General Relativity (SILARG VII). Proceeding appeared as "Relativity and Gravitation: Classical and Quantum", edited by J. D'Olivio et al (World Scientific, Singapore, 1991); Y. Zhang, Ph.D thesis (University of Maryland, 1990);

E. Calzetta, B. L. Hu and F. D. Mazzitelli, "Coarse-grained effective action and renormalization group theory in semiclassical gravity and cosmology", Phys. Rep. 352, 459 (2001).

[24] P. R. Johnson and B. L. Hu, "Stochastic theory of relativistic particles moving in a quantum field: scalar Abraham-Lorentz-Dirac-Langevin equation, radiation reaction, and vacuum fluctuations", Phys. Rev. D 65, 065015 (2002).

[25] C. R. Galley and B. L. Hu, "Self-force with a stochastic component from radiation reaction of a scalar charge moving in curved spacetime", Phys. Rev. D 72, 084023 (2005).

[26] E. Calzetta, A. Roura and E. Verdaguer, "Stochastic description for open quantum systems", Physica A 319, 188 (2003).

[27] J.-T. Hsiang and B. L. Hu, "Nonequilibrium nonlinear open quantum systems I: Functional perturbative analysis of a weakly anharmonic oscillator", Phys. Rev. D (2020); [arXiv:1912.12803].

[28] J.-T. Hsiang and B. L. Hu, "Fluctuation-dissipation relation from the nonequilibrium dynamics of a nonlinear open quantum system", Phys. Rev. D (2020); [arXiv:2002.07694].

[29] B. L. Hu, J. P. Paz and Y. Zhang, "Quantum Brownian motion in a general environment. II. nonlinear coupling and perturbative approach", 47, 1576 (1993).

[30] F. Bonetto, J. L. Lebowitz and L. Rey-Bellet, "Fourier law: a challenge to theorists" in Mathematical Physics 2000, (Imperial College Press, London 2000); [arXiv:math-ph/0002052].

[31] S. Lepri, R. Livi and A. Politi, "Heat conduction in chains of nonlinear oscillators", Phys. Rev. Lett. 78, 1869 (1997); "On the anomalous thermal conductivity of one-dimensional lattices", Europhys. Lett. 43, 271 (1998).

[32] S. Lepri, R. Livi and A. Politi, “Thermal conduction in classical low-dimensional lattices”, Phys. Rep. 377, 1 (2003).

[33] A. Dhar, "Heat transport in low-dimensional systems", Adv. Phys. 57, 457 (2008).

[34] N. Li, J. Ren, L. Wang, G. Zhang, P. Hänggi and B. Li, "Phononics: Manipulating heat flow with electronic analogs and beyond", Rev. Mod. Phys. 84, 1045 (2012). 
[35] A. Dhar, K. Saito and P. Hänggi, "Nonequilibrium density-matrix description of steady-state quantum transport", Phys. Rev. E 85, 011126 (2012).

[36] L. P. Kadanoff and G. Baym, "Quantum Theory of Many-Particle Systems", (Dover, 1962).

[37] J. Rammer, "Quantum Field Theory of Non-equilibrium States" (Cambridge University Press, Cambridge, 2007).

[38] J. Maciejko, J. Wang, and H. Guo, "Time-dependent quantum transport far from equilibrium: An exact nonlinear response theory", Phys. Rev. B 74, 085324 (2006).

[39] J.-S.Wang, B. K. Agarwalla, H. Li and J. Thingna, "Nonequilibrium Green's function method for quantum thermal transport", Front. Phys. 9, 673 (2014).

[40] Pilgram, S., Jordan, A.N., Sukhorukov, E.V. and Büttiker, M., 2003. Stochastic path integral formulation of full counting statistics. Physical Review Letters, 90(20), p.206801.

[41] Jordan, A.N., Sukhorukov, E.V. and Pilgram, S., 2004. Fluctuation statistics in networks: A stochastic path integral approach. Journal of mathematical physics, 45(11), pp.4386-4417.

[42] J. S. Schwinger, "Brownian motion of a quantum oscillator", J. Math. Phys. (N.Y.) 2, 407 (1961);

L. Keldysh, "Diagram technique for nonequilibrium processes", Zh. Eksp. Teor. Fiz. 47, 1515 (1964); [JETP 20, 1018 (1964)];

K.-C. Chou, Z.-B. Su, B.-L. Hao and L. Yu, "Equilibrium and non-equilibrium formalisms made unified", Phys. Rep. 118, 1 (1985).

[43] E. A. Calzetta and B.-L. B. Hu, "Nonequilibrium Quantum Field Theory", (Cambridge University Press, Cambridge, 2008).

[44] A. Kamenev, "Field Theory of Non-equilibrium Systems", (Cambridge University Press, Cambridge, 2011).

[45] U. Weiss, "Quantum Dissipative Systems, 4th Edition". (World Scientific, New Jersey, 2012).

[46] R. P. Feynman and F. L. Vernon, "The theory of a general quantum system interacting with a linear dissipative system ", Ann. Phys. (N.Y.) 24, $118(1963)$

A. O. Caldeira and A. J. Leggett, "Path integral approach to quantum Brownian motion", Physica A 121, 587 (1983);

H. Grabert, P. Schramm and G. L. Ingold, "Quantum Brownian motion, the functional integral approach", Phys. Rep. 168, 115 (1988).

[47] B. L. Hu, J. P. Paz and Y. Zhang, "Quantum Brownian motion in a general environment: exact master equation with nonlocal dissipation and colored noise", Phys. Rev. D 45, 2843 (1992).

[48] H. Zhao, Z. Wen, Y. Zhang and D. Zheng, "Dynamics of solitary wave scattering in the Fermi-Pasta-Ulam model”, Phys. Rev. Lett. 94,025507 (2005);

H. Zhao, "Identifying diffusion processes in one-dimensional lattices at thermal equilibrium", Phys. Rev. Lett. 96, 140602 (2006);

S. Chen, Y. Zhang, J. Wang and H. Zhao, "Diffusion of heat, energy, momentum and mass in one-dimensional systems", Phys. Rev. E 87, 032153 (2013);

H. Zhao and H. Zhao, "Brownian motion: from kinetics to hydrodynamics", [arxiv:1706.00779];

D. Xiong, "Heat perturbation spreading in the Fermi-Pasta-Ulam-beta system with next-nearest-neighbor coupling: Competition between phonon dispersion and nonlinearity", Phys. Rev. E 95, 062140 (2017).

[49] H. Spohn, "The phonon Boltzmann equation, properties and link to weakly anharmonic lattice dynamics", J. Stat. Phys. 124, 1041 (2006).

[50] C. B. Mendl and H. Spohn, "Dynamic correlators of Fermi-Pasta-Ulam chains and nonlinear fluctuating hydrodynamics", Phys. Rev. Lett. 111, 230601 (2013);

H. Spohn, "Nonlinear fluctuating hydrodynamics for anharmonic chains", J. Stat. Phys. 154, 1191 (2014);

S. G. Das, A. Dhar, K. Saito, C. B. Mendl and H. Spohn, "Numerical test of hydrodynamic fluctuation theory in the Fermi-Pasta-Ulam chain", Phys. Rev. E 90, 012124 (2014);

C. B. Mendl and H. Spohn, "Current fluctuations for anharmonic chains in thermal equilibrium”, J. Stat. Mech. 2015 , P03007 (2015).

[51] A. Dhar, private communication 2014;

A. Dhar and K. Wagh, "Equilibration problem for the generalized Langevin equation”, Europhys. Lett. 79, 60003 (2007).

[52] H. Zhao, private communication 2014.

[53] W. Fu, Y. Zhang and H. Zhao, “Universal scaling of the thermalization time in one-dimensional lattices”, Phys. Rev. E 100, 010101(R) (2019).

[54] Z. Wang, W. Fu, Y. Zhang and H. Zhao, "Arbitrarily weak nonlinearity can destroy the Anderson localization”; [arXiv:1903.09502v2].

[55] L. Pistone, M. Onorato and S. Chibbaro, "Thermalization in the discrete nonlinear Klein-Gordon chain in the wave-turbulence framework", Euro. Phys. Lett. 12144003 (2018).

[56] L. Pistone, S. Chibbaro, M. D. Bustamante, Y. L’vov and M. Onorato, "Universal route to thermalization in weakly-nonlinear one-dimensional chains", Mathematics in Engineering 1, 672 (2019); [arXiv:1812.08279].

[57] A. Chantasri, J. Dressel, and A. N. Jordan. "Action principle for continuous quantum measurement." Physical Review A 88.4 (2013): 042110.

[58] A. Chantasri, and A. N. Jordan. "Stochastic path-integral formalism for continuous quantum measurement." Physical Review A 92.3 (2015): 032125 .

[59] J. T. Stockburger and C. H. Mak, "Stochastic Liouvillian algorithm to simulate dissipative quantum dynamics with arbitrary precision" J. Chem. Phys. 110, 4983 (1999).

[60] Léger, S., Puertas-Martínez, J., Bharadwaj, K., Dassonneville, R., Delaforce, J., Foroughi, F., ... \& Hasch-Guichard, W. (2019). Observation of quantum many-body effects due to zero point fluctuations in superconducting circuits. Nature communications, 10(1), 1-8.

[61] Kuzmin, R., Mencia, R., Grabon, N., Mehta, N., Lin, Y. H., \& Manucharyan, V. E. (2019). Quantum electrodynamics of a superconductorinsulator phase transition. Nature Physics, 15(9), 930-934.

[62] Martinez, Esteban A., and Juan Pablo Paz. "Dynamics and thermodynamics of linear quantum open systems.” Physical review letters 110.13 (2013): 130406. 